\title{
THEORY AND PRACTICE OF MODULATED TEMPERATURE DIFFERENTIAL SCANNING CALORIMETRY
}

\author{
ANDREW A. LACEY ${ }^{1}$, DUNCAN M. PRICE ${ }^{2} \&$ MIKE READING $^{2}$ \\ 1. Department of Mathematics, Heriot-Watt University, Riccarton, Edinburgh \\ EH14 4AS UK. \\ 2. Institute of Polymer Technology \& Materials Engineering, Loughborough \\ University, Loughborough LE11 3TU UK.
}

\section{Introduction}

There are two different literatures on the subject of DSC and calorimetry in general; the first deals mainly with its applications, the second primarily with the technique itself. The latter includes, amongst other things, commentary on instrument calibration, the limits of sensitivity and resolution, the details of modelling the response of the calorimeter and separating the effects of the measuring system from those due to the phenomenon being studied. Certainly there is overlap between these two bodies of work. However, it is also true that it is not necessary to understand fully the details of the equations that can be used to model heat flow in a DSC cell in order to measure and interpret a glass transition successfully. In this book we attempt to strike a balance between satisfying both audiences. In this chapter, in particular, we attempt, in the first part, to provide sufficient information to enable the polymer scientist to interpret correctly his or her results while not burdening the reader with details that might ultimately obscure the central meaning. This is intended for those more interested in the results themselves than the process by which they are derived. There is a discussion of theory, but this is confined to the important results rather than the details of their derivation. In the second part, a more extended discussion is offered on the considerable complexities of understanding the details of modulated temperature calorimetry in its modern form (i.e. an experiment where both the response to the modulation and underlying heat flow are both obtained simultaneously and compared for a wide range of transitions). The first part is called "Practical MTDSC", the second "Detailed Discussion of the Theory of MTDSC".

It is not the intention of this chapter to be a review of the literature (if the reader is looking for this [1] is a recent example). Its purpose is to serve as an introduction to the technique of MTDSC starting with fairly basic and practical matters then progressing onto more advanced levels. It is also intended to serve as a guide to understanding the remaining chapters that deal with the three principal classes of polymeric materials, thermosets, thermoplastic polymer blends and semi-crystalline polymers.

The use of a modulated temperature profile with DSC, combined with a deconvolution procedure in order to obtain the same information as conventional DSC plus, at the same time, the response to the modulation, was first proposed by Reading and co-workers [2-17]. In this 
section we will describe the basic deconvolution procedure i.e. how that data is processed and presented for a typical polymer sample. We then consider how these data are interpreted.

\section{The Basics of Modulated Temperature Differential Scanning Calorimetry}

\subsection{SOME PRELIMINARY OBSERVATIONS ON HEAT CAPACITY}

Heat capacity can be defined as the amount of energy required to increase the temperature of a material by 1 degree Kelvin or Celcius. Thus,

$C_{p}=Q / \Delta T$

where $C_{p}=$ the heat capacity

$\Delta T=$ the change in temperature

$Q=$ amount of heat required to achieve $\Delta T$

Often it would be considered that this is the heat stored in the molecular motions available to the material, that is the vibrational, translational motions etc. It is stored reversibly. Thus the heat given out by the sample when it is cooled by $1^{\circ} \mathrm{C}$ is exactly the same as that required to heat it by the same amount. This type of heat capacity is often called vibrational heat capacity. Where temperature is changing, the rate of heat flow required to achieve this is given by:

$d Q / d t=C_{p} d T / d t$

where $t=$ time

This is intuitively obvious. Clearly if one wishes to increase the temperature of the material twice as fast, twice the amount of energy per unit time must be supplied. If the sample has twice the heat capacity, this also doubles the amount of heat required per unit time for a given rate of temperature rise. Considering a linear temperature programme, such as is usually employed in scanning calorimetry:

$T=T_{0}+\beta t$

where $T=$ temperature

$T_{0}=$ starting temperature

$\beta=$ the heating rate $d T / d t$ 
This leads to:

$d Q / d t=\beta C_{p}$

or

$C_{p}=(d Q / d t) / \beta$

This provides one way of measuring heat capacity in a linear rising temperature experiment: one simply divides the heat flow by the heating rate. If the temperature programme is replaced by one comprising a linear temperature ramp modulated by a sine wave, this can be expressed as:

$T=T_{0}+\beta t+B \sin \omega t$

where $B=$ the amplitude of the modulation

$\omega=$ the angular frequency of the modulation

The derivative with respect to time of this is

$d T / d t=\beta+\omega B \cos \omega t$

Thus, it follows:

$d Q / d t=C_{p}(\beta+\omega B \cos \omega t)$

For the special case where $\beta$ is zero, this yields:

$d Q / d t=C_{p} \omega B \cos \omega t$

For the simplest possible case, from equation 2, the resultant heat flow must also be a cosine wave thus:

$A_{H F} \cos \omega t=\mathrm{C}_{\mathrm{p}} \omega B \cos \omega t$

where $A_{H F}=$ the amplitude of the heat flow modulation

It follows that $\omega B=$ the amplitude of the modulation in the heating rate. Thus:

$C_{p}=\mathrm{A}_{\mathrm{HF}} / \mathrm{A}_{\mathrm{HR}}$ 
where $A_{H R}=$ amplitude of modulation in heating rate $(=\omega B)$.

This provides a second method of measuring heat capacity, by looking at the amplitude of the modulation. The same relationship applies even if there is an underlying heating ramp. In essence, MTDSC is based on simultaneously measuring the heat capacity of the sample using both methods, the response to the linear ramp and the response to the modulation, and comparing them. When the sample is inert and there are no significant temperature gradients between the sample temperature sensor and the centre of the sample, both methods should give the same value. The interest lies in the fact that during transitions, these two methods give different values.

\subsection{THE MTDSC EXPERIMENT AND DECONVOLUTION PROCEEDURE}

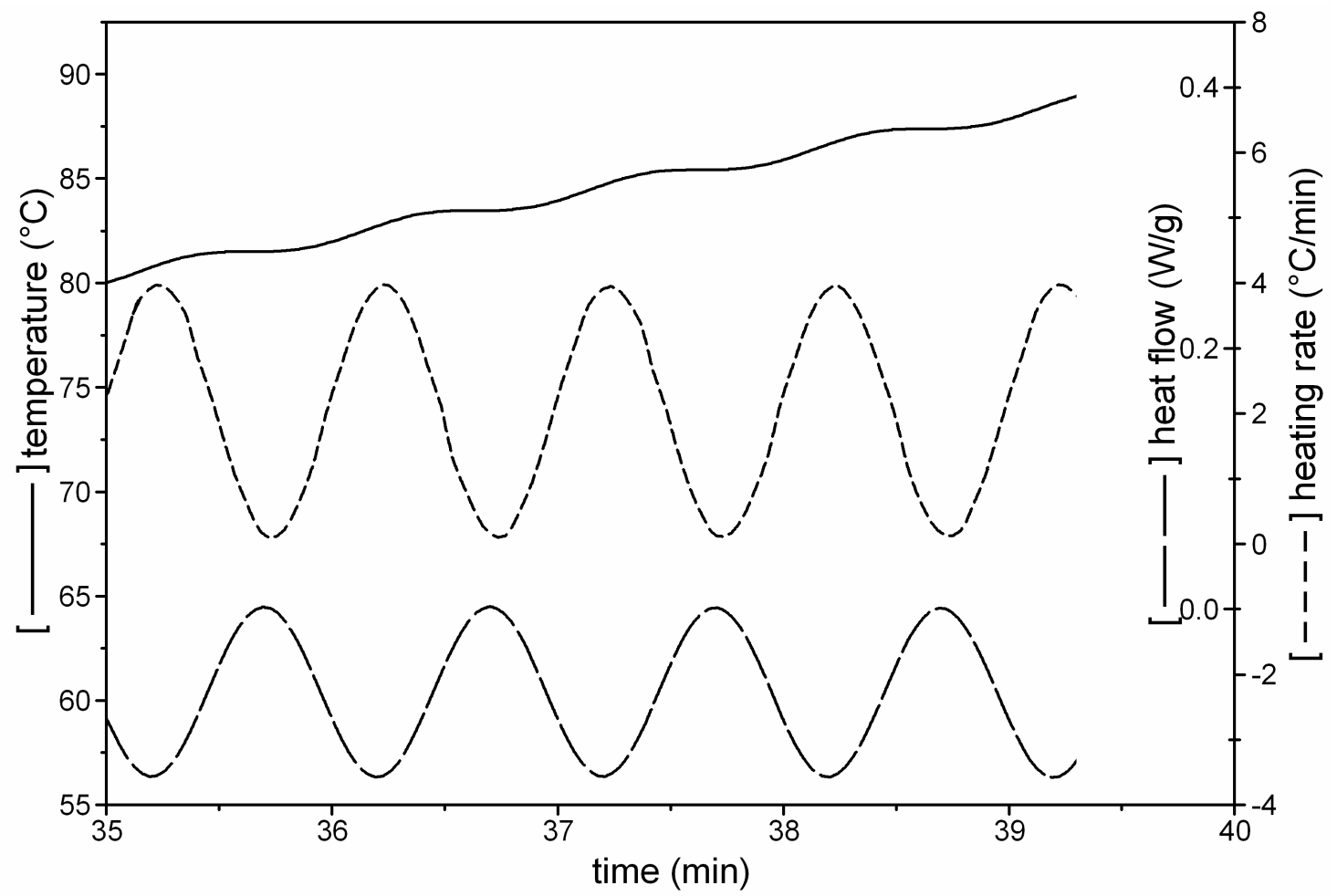

Fig. 1 Typical temperature-time curve for an MTSC experiment (top) with resultant heating rate modulation and heat flow response (underlying heating rate: $2^{\circ} \mathrm{C} /$ min, period 60s, amplitude: $0.318^{\circ} \mathrm{C}$, under nitrogen).

Although many different forms of temperature programme are possible, a sinusoidal temperature modulation is most often used, as illustrated in Fig. 1. Fig. 2 shows data for amorphous poly(ethylene terephthalate), PET, from below its glass-rubber transition temperature $\left(T_{g}\right)$ to above its melting temperature. The modulation in heating rate and the resultant heat flow is shown as well as one of the signals derived from the deconvolution 
procedure, the phase lag between the modulation in the heating rate and that in the heat flow. As the first step in the deconvolution process, the raw data are averaged over the period of one oscillation to remove the modulation. This then gives the total signal, which is equivalent to the signal that would have been obtained had the modulation not been used, i.e. a conventional DSC experiment (see below). The averaged signal is subtracted from the raw data and the modulation is then analysed using a Fourier transform procedure to obtain the amplitude and phase difference of the heat flow response at the frequency of the imposed modulation.

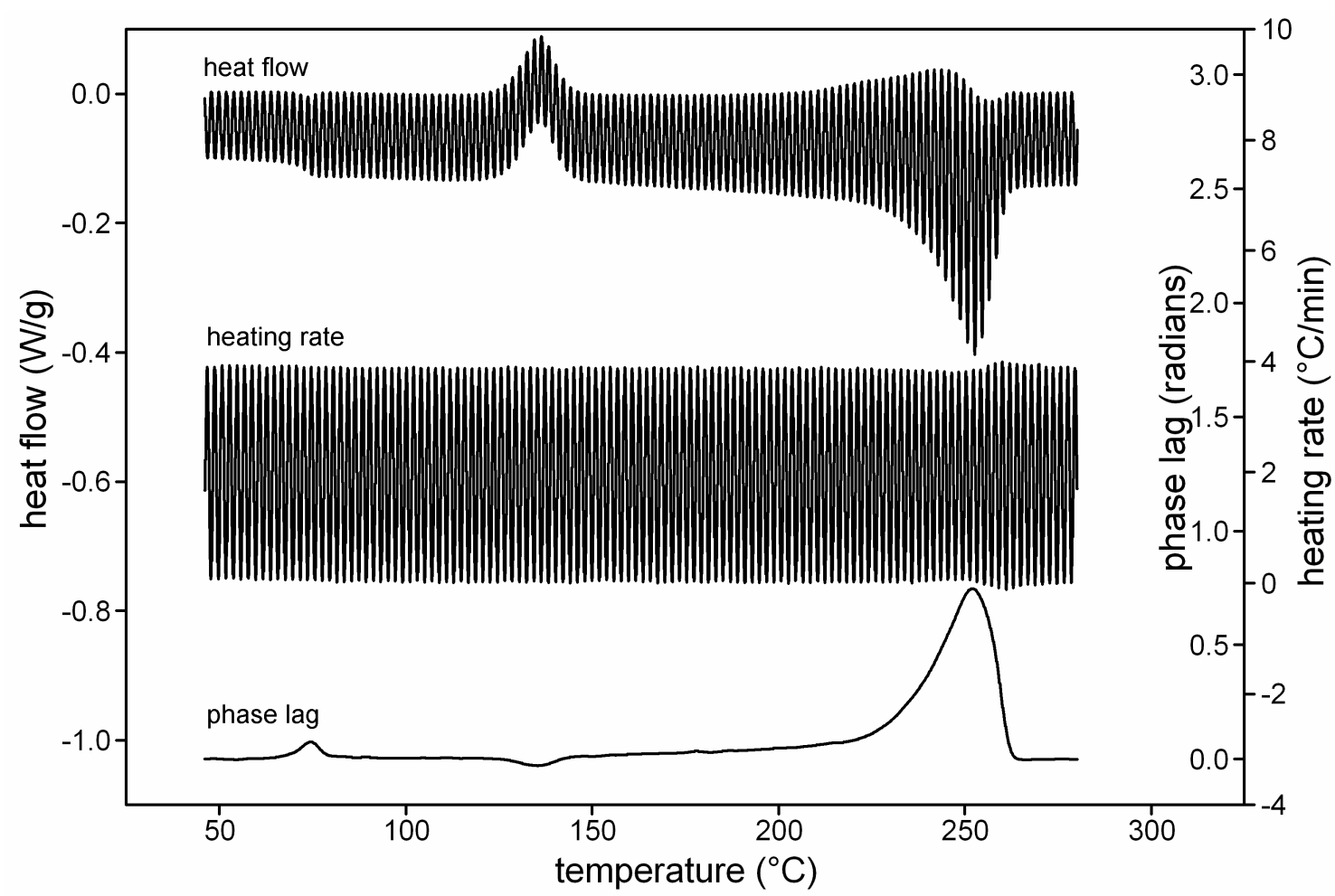

Fig. 2 Raw data from a MTDSC experiment for quenched PET plus one signal resulting from the Fourier transform, the phase lag (underlying heating rate: $2^{\circ} \mathrm{C} / \mathrm{min}$, period 60s, amplitude: $0.318^{\circ} \mathrm{C}$, under nitrogen).

In contrast to the very simple treatment outlined above in section 2.1, one can allow for the situation that the heat flow modulation might not always follow exactly the cosine modulation in the heating rate (for reasons that will become clear when in the discussions on various transitions), thus the heat flow may lag behind:

The heat flow modulation $=A_{H F} \cos (\omega t-\phi)$

where $\phi=$ the phase difference between the modulation in the heat flow and the heating rate, also termed the "phase lag". 
The basic output from the first stage of the deconvolution procedure is therefore

$\langle d Q / d t\rangle=$ the average or total heat flow

where $<>$ denotes the average over one or more periods.

$Q$ = heat,

$A_{H F}=$ amplitude of the heat-flow modulation,

$A_{H R}=$ amplitude of modulation in the heating rate,

$\phi=$ the phase lag.

Having obtained the amplitudes of the modulations in heating rate and heat flow the next step is to use these quantities to calculate a value for the heat capacity as in equation 11. Viz;

$A_{H F} / A_{H R}=C^{*}$

where $C^{*}=$ the reversing heat capacity (also called the cyclic heat capacity or modulus of complex heat capacity. See below)

There are then two alternative ways of proceeding with the deconvolution - both of which were originally proposed by Reading and co-workers [2-5];

\subsubsection{The Simple Deconvolution Procedure}

If the results are to be expressed as heat capacities then the average total heat flow is divided by the underlying heating rate $\beta$. Thus

$\left\langle d Q / d t>/ \beta=C_{p T}=\right.$ the average or total heat capacity

Having obtained the reversing heat capacity and one can calculate the non-reversing heat capacity. Viz

$<d Q / d t>/ \beta-C^{*}=$ the non-reversing heat capacity $=C_{p N R}$ 


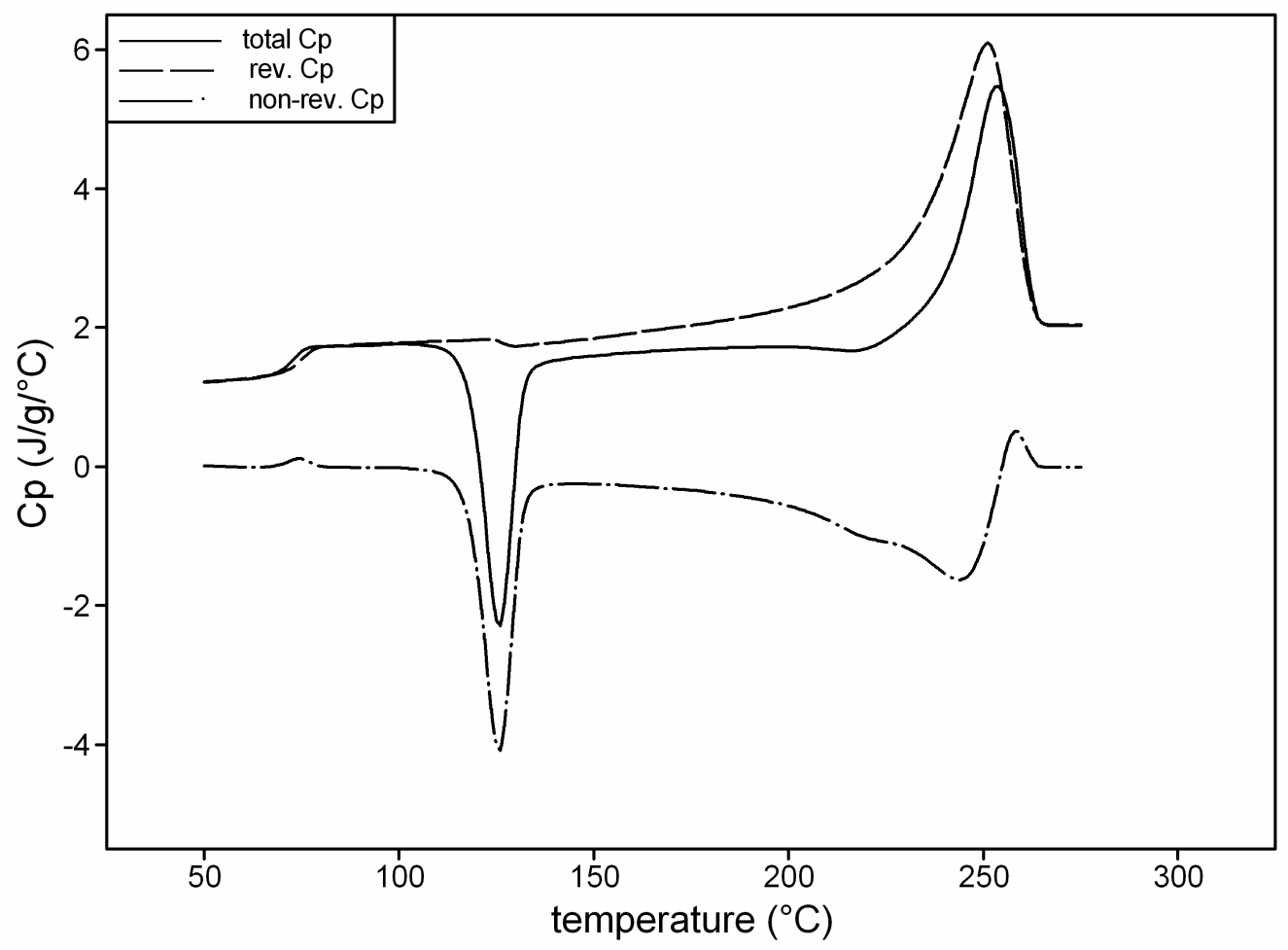

Fig. 3 Results from simple deconvolution procedure for the data shown in Fig. 2.

This is illustrated in Fig. 3 using the data shown in Fig. 2. Note that in non-transition regions, for example below the glass transition and in the molten state, the reversing and total heat capacities are the same. As should be clear from the discussion in section 2.1, and the theoretical arguments advanced below, this is exactly what we would expect. If measurements were made on an inert material such as sapphire, then the reversing and total signals should be coincident and the reversing signal would be zero. However, all measurements contain errors and so exact agreement is difficult to achieve. It requires careful calibration (see below) and good experimental practice. Where there are minor discrepancies, it is useful to use nontransition regions as a kind of internal calibration and use a linear baseline correction such as is illustrated in Fig. 4. The two signals are forced to be the same where it is known that they should be. Whether the total or the reversing heat capacity is taken to give the 'correct' value is a matter of judgement given the experimental conditions used (and may well be irrelevant depending on what information is being sought from the experiment). This is discussed in the sections dealing with selection of experimental conditions and calibration below. The nonreversing signal is calculated after any shift to make the non-transition reversing and total signals the same. 


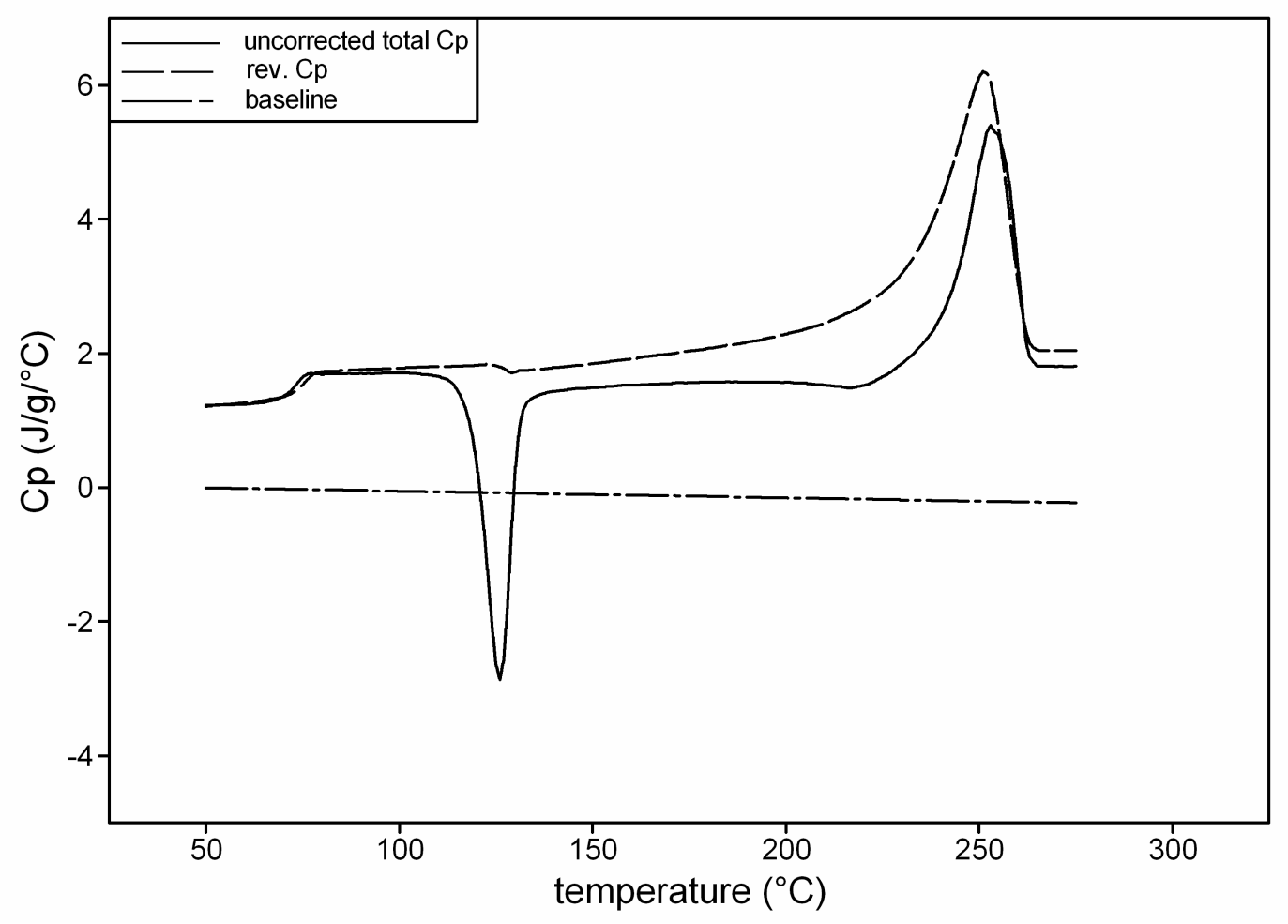

Fig. 4 Total and reversing $C_{p}$ before baseline correction.

It can be argued that enthalpies associated with, for example, crystallisation, should not be expressed as changes in heat capacity in the way shown in Fig. 3. Perhaps this term is best reserved for the reversible storage of heat in the motions of the molecules such as we see in the non-transition regions. This is a moot point. In practice, results are often expressed in terms of heat capacity, regardless of any transitions that occur, and this convention is followed in this book.

Although all of these signals in Fig. 3 are expressed as heat capacities, they can equally well be expressed as heat flows:

$\langle d Q / d t\rangle=$ average or total heat flow

$C^{*} \beta=$ reversing heat flow

This is then subtracted from the total heat flow to obtain the non-reversing heat flow. Viz.:

$<d Q / d t>-C^{*} \beta=$ non-reversing heat flow

The convention often adopted for heat flux DSC's means that exotherms go up and so, in addition to changing the units on the y-axis, expressing the signals as heat flows also sometimes 
means inverting the curves compared to Fig. 3. However, it is not uncommon to express exotherms as going down even when plotting the data as heat flow. The reader simply needs to be careful in regard to what units and conventions are being used.

Note that the simple deconvolution procedure makes no use of the phase lag signal.

\subsubsection{The Complete Deconvolution Procedure}

In this procedure the phase lag is used to calculate the in- and out-of-phase components of the cyclic heat capacity. Viz:

$C^{*} \cos \phi=$ phase-corrected reversing heat capacity $=C_{P P C R}$

$C^{*} \sin \phi=$ kinetic heat capacity $=C_{p K}$

In reality, the phase angle cannot usually be used directly, a baseline correction is required. This is dealt with in the calibration section below.

The complete deconvolution then proceeds in the same way as for the simple deconvolution except that the phase-corrected reversing heat capacity is used instead of the reversing heat capacity thus:

$<d Q / d t>/ \beta-C_{p P C R}=$ the phase-corrected non-reversing heat flow $=C_{p P C N R}$

The results of this deconvolution applied to the data in Fig. 2 are given in Fig. 5.

Again, all of the signals can also be expressed as heat flows

$\langle d Q / d t>=$ average or total heat flow

$C_{P P C R} \beta=$ phase-corrected reversing heat flow

$<d Q / d t>-C_{p P C R} \beta=$ phase-corrected non-reversing heat flow

$C_{p K} \beta=$ kinetic heat flow

Thus, in general, all signals can all equally well be expressed as heat capacities or heat flows simply by multiplying or dividing by the underlying heating rate $\beta$ as appropriate. Often both types of signals are mixed, so reversing heat capacity is co-plotted with non-reversing heat flow. 


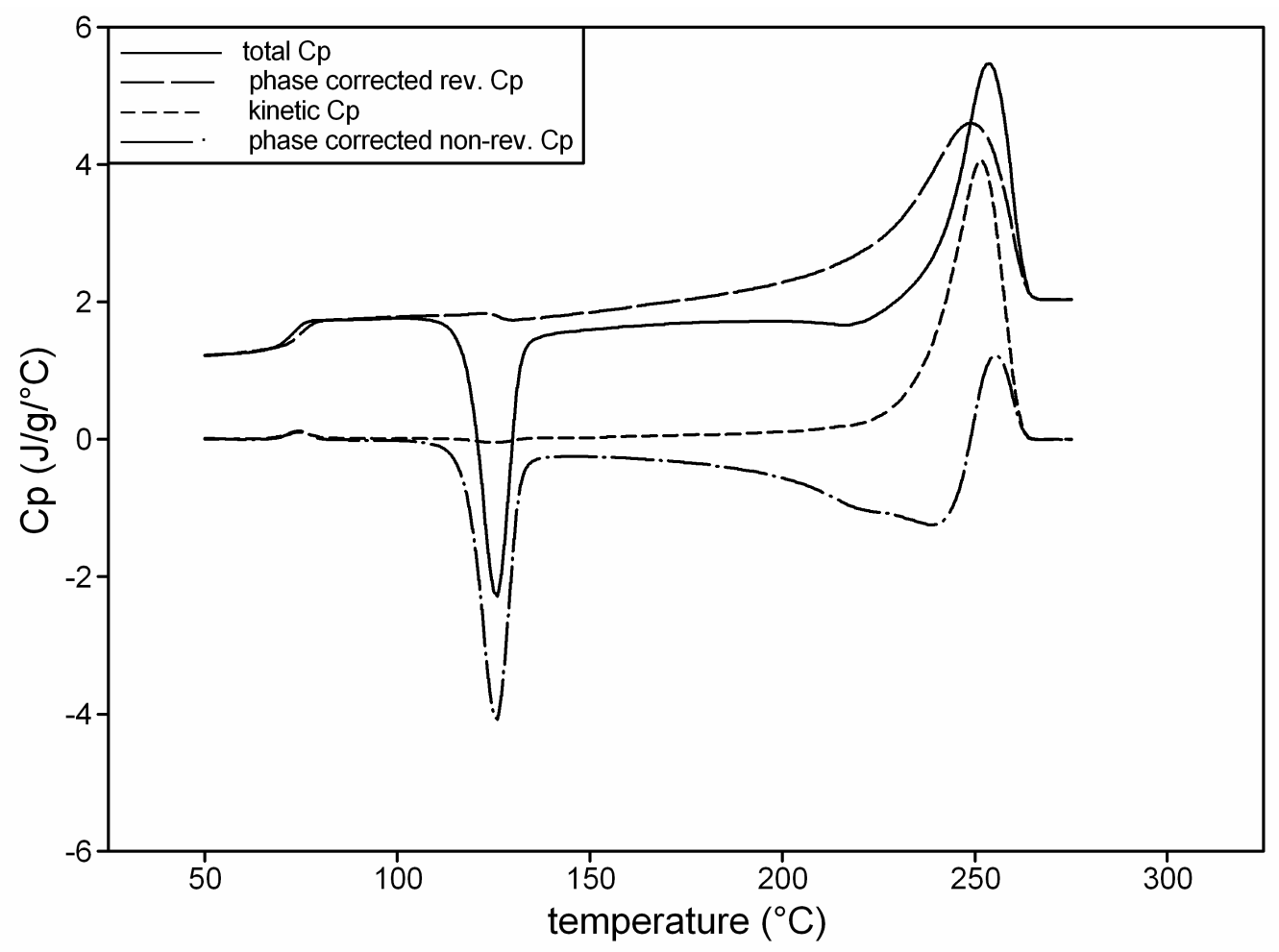

Fig. 5 Results of complete deconvolution procedure for the data shown in Fig. 2.

\subsubsection{Comments on the Different Deconvolution Procedures}

In Fig. 6, a comparison is made between the reversing and the phase-corrected reversing and non-reversing signals. It can be seen that there is only a significant difference in the melt region. In reality the simple deconvolution is an approximate form of the complete deconvolution procedure. The phase correction is, in most polymer transitions except melting, negligible, as illustrated in Fig. 6. Thus:

$C^{*} \approx C_{p P C R}$

A quantitative interpretation of results in the melt region, with or without the use of the phase lag, is often problematic. As a consequence of this, it often does not matter whether the phase correction has been applied or not unless the kinetic heat flow is specifically of interest. In many of the applications described in this book no phase correction has been used. However, it must be stressed that there is no conflict between the simple and complete deconvolution procedures. Perhaps because of an initial misunderstanding in the literature $[2,15,17,18]$, even today authors present the deconvolution into reversing and non-reversing as an alternative to using the phase correction (to derive the phase corrected reversing and kinetic heat capacities or complex heat capacity, see below). It is sometimes even presented as a rival method. This 
confusion in the literature is an obstacle to a proper understanding of the technique and it is to be hoped that in future it will cease. The use of the phase lag is an optional refinement that has always been part of MTDSC from the time it was first introduced [2]. The full deconvolution does provide the maximum information and workers who prefer this are encouraged to pursue it. If it is not used routinely, it is simply because the phase lag is sensitive to non-ideal behaviour of the combination of the sample, pan and measuring system and correcting for this requires additional effort (see the calibration section below) often with little practical benefit. However, improvements in instrumentation and software will probably make the full deconvolution routine in future.

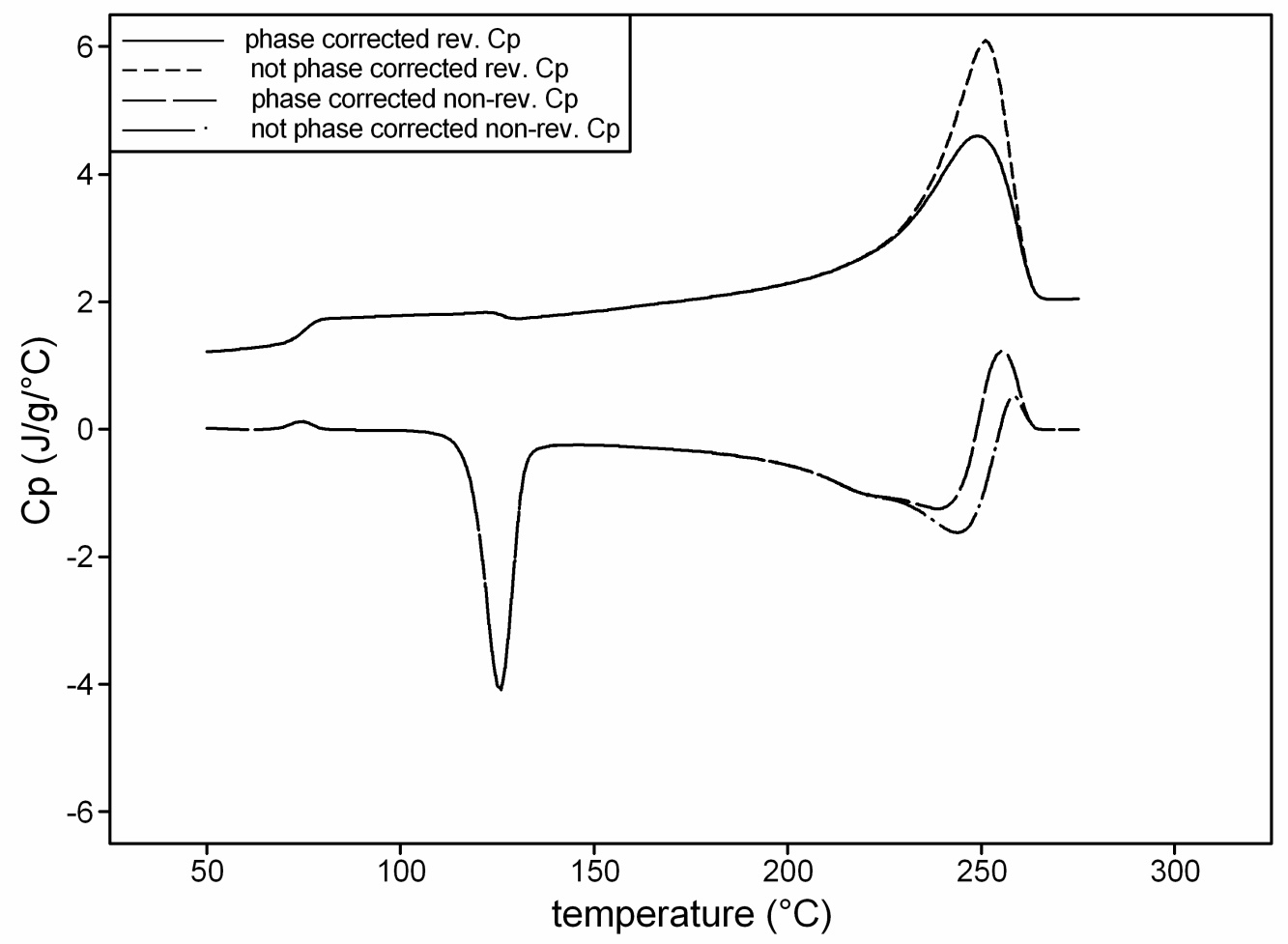

Fig. 6 Co-plot of reversing and non-reversing heat capacity arising from the simple and full deconvolution procedures applied to the raw data from Fig. 2.

\subsubsection{Comments on Nomenclature}

The reason for the nomenclature reversing and non-reversing will be given below as part of the discussion on practical MTDSC in the section on chemical reactions and related processes. It was the original intention of Reading and co-workers that the term reversing should mean what is referred to above as the phase-corrected reversing [19] while accepting that in most cases the phase lag correction would not be used because it is very small. However, the de facto 
current practice is that reversing applies to the non-phase-corrected signal and this is the convention that we use in this book.

It is also possible, and often helpful, to use complex notation. The ratio of the amplitudes of the modulations of the temperature rise and heat flow gives one useful piece of information: $C^{*}=A_{H F} / A_{H R}$. The phase lag gives another. These two bits of information are equivalent to knowing both $C_{p P C R}$ and $C_{P K}$, or the single complex quality $\hat{C}=C_{p R}-i C_{P K}$ where $i=$ the square root of -1 . Since the temperature-rise and heat-flow modulations can be written as $\operatorname{Re}\left\{\omega B e^{i \omega t}\right\}$ and $\operatorname{Re}\left\{A_{H F} e^{i(\omega t-\phi)}\right\}=\operatorname{Re}\left\{\left(A_{H F} \cos \phi-i A_{H F} \sin \phi\right) e^{i \omega t}\right\}$ respectively $\left(A_{H R}=\omega B\right)$, the complex heat capacity can be defined directly.

$\hat{C}=\frac{A_{H F} e^{-i \phi}}{A_{H R}}=C^{\prime}-i C^{\prime \prime}$

where $C_{P P C R}=C^{\prime}=$ the real component, $C_{p K}=C^{\prime \prime}=$ the imaginary component.

Manipulations needed to relate heat flow $A_{H R}$ to temperature changes through theoretical models for transitions, or through properties of calorimeters, are usually more conveniently done via such complex qualities. The value $\hat{C}$ can then lead directly to evaluations of real specific heat and parameters controlling kinetics. However, the use of complex notation does not imply a different theoretical treatment or method. It is simply a more convenient mathematical formalism. The terms 'real' and 'imaginary' heat capacity and 'phase-corrected reversing' and 'kinetic' heat capacities are interchangeable.

It is regrettable that such a proliferation of names is in common use and this must be confusing to many workers. However, by paying close attention to the above text, it should be possible to deduce the correct signal in almost all cases.

\section{Practical Modulated Temperature DSC}

\subsection{THE IMPORTANCE OF LINEARITY}

One point that needs to be mentioned is that the analysis described above assumes that the sample's response to the modulation can be approximated as linear. Clearly, the processes such as those that follow Arrhenius kinetics or the related kinetics of a glass transition are not linear with temperature. However, over a small temperature interval they can be approximated as linear. Where this cannot be said to be true, the above analysis fails because it assumes a linear response. 
Where a multiplexed sine wave or saw-tooth modulation is used the deconvolution procedure can be used to extract the response at a series of frequencies $[4,10,19,20]$. However, current commercial products restrict themselves to using the first component of the Fourier series, which is then, with the assumption of linearity, equivalent to using a single sinusoidal modulation. It is true that looking at the whole Fourier series, rather than just the first component, offers scope for increasing the amount of information that can be obtained from a MTDSC experiment. This applies even to single sinusoidal modulations (because nonlinearities produce harmonics) as well as multiple simultaneous sine waves or saw-tooth modulations. This will be considered in greater detail below in the section on advanced MTDSC.

\subsection{SELECTION OF EXPERIMENTAL PARAMETERS}

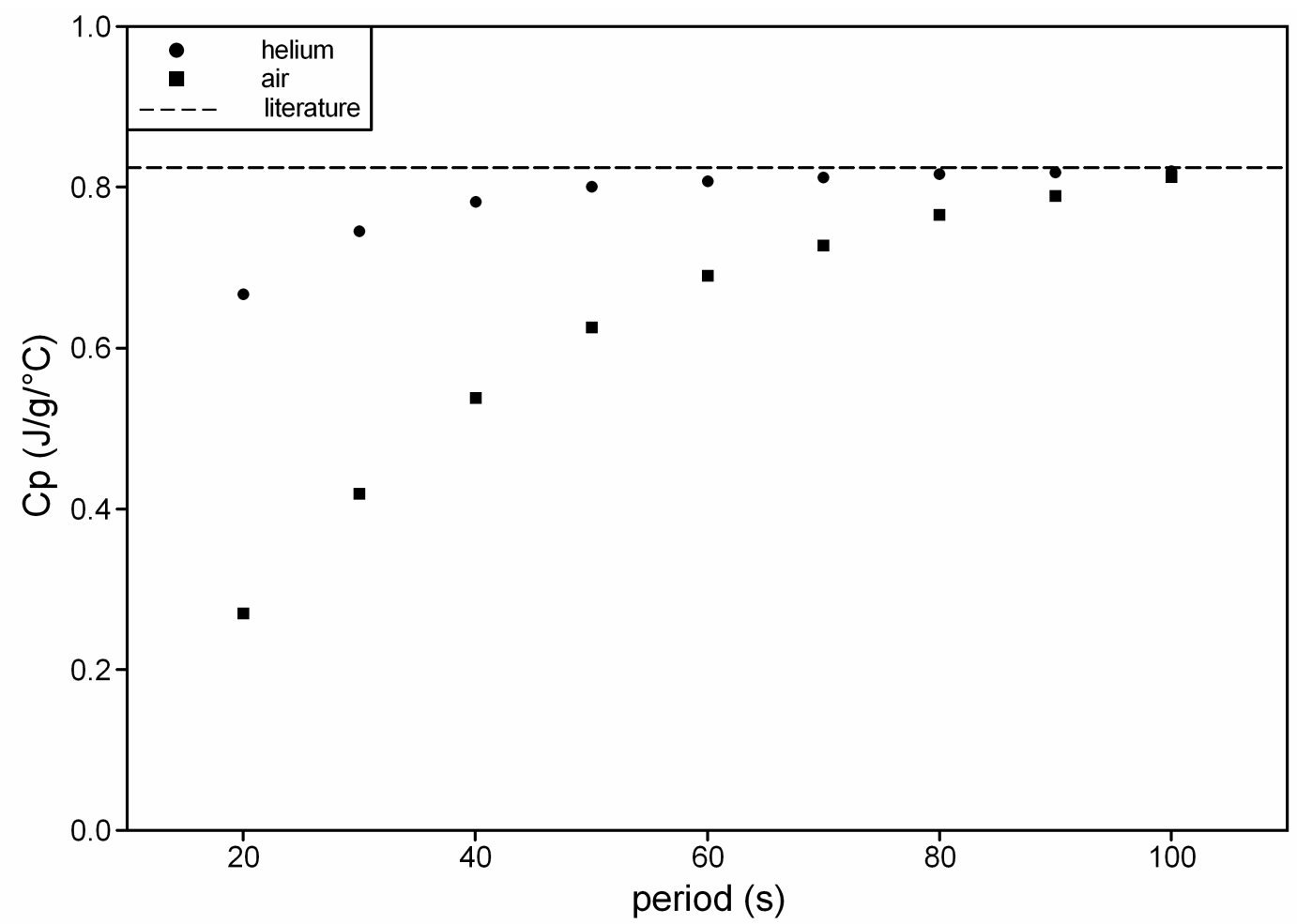

Fig. 7 Reversing $C_{p}$ for sapphire as a function of period in helium and air (quasiisothermal measurement at $50^{\circ} \mathrm{C}$, amplitude $1^{\circ} \mathrm{C}$ ).

A fundamental consideration that always applies is the requirement that there be many modulations over the course of any transition. Stated simply, the deconvolution procedure described above can only make sense if the underlying heat flow is changing slowly and smoothly under the modulation. If this is true, averaging the modulated signal over the over the period of the modulation will provide the same information as an un-modulated experiment to 
a good approximation. The averaging will usually mean the modulated experiment looks 'smoothed' to some extent thus the tops of peaks may be a little 'rounder' but the areas under peaks and all of the essential features will be the same. If a significant part of a transition occurs over the course of a single modulation, this invalidates the assumptions behind the use of the Fourier series. As the reader proceeds through the sections on theory and typical results, it is hoped that these points will become intuitively obvious. As a general rule of thumb for most polymer applications, where the transition is a peak in $d Q / d t$, then there should be at least 5 modulations over the period represented by the width at half height. Where the transition is a step change, there should be at least 5 modulations over that part of the transition where change is most rapid. Where there is doubt, the number of modulations should be increased by reducing the underlying temperature ramp to check whether this significantly changes the reversing signal.

There is the question of what period should be used. As mentioned above, for an inert material the reversing heat capacity should provide an accurate measure of the specific heat capacity (= heat capacity / mass) of the sample when the calorimeter is calibrated in the conventional way (see below). This is true when the period is long, typically over 100 seconds or more. As the period becomes shorter, the apparent reversing heat capacity becomes smaller as illustrated in Fig. 7. This happens because there are thermal resistances between the pan and the temperature sensor, the pan and the sample and within the sample itself. A long period implies a slow underlying heating rate that is undesirable because this means a long time for the experiment and a reduction in the signal to noise in the total signal. A typical compromise is 60 seconds used with a calibration factor determined using a calibrant with an accurately known heat capacity (this is described in the calibration sections below). In Fig. 7 it can be seen that the effects of the thermal resistances are smaller when helium is used and a reasonable compromise is 40 seconds (again with a calibration factor). While it is true that considerable progress has been made by some workers in characterising and compensating for these nonideal experimental conditions [12, 21-25] for most experimentalists, the best approach it to use longer periods that avoid the complications engendered by these thermal resistances. It should be noted that, if helium is used, the concentration of helium in the actual cell will generally not be $100 \%$ and will vary with flow rate. This means that the flow rate must be accurately controlled (usually with a mass flow controller).

Once the period is chosen, the requirement that there be many modulations over the course of all transitions then sets limits on the maximum heating rate that can be used. A typical heating rate with a 60 -second period would be $2^{\circ} \mathrm{C} / \mathrm{min}$. or $3^{\circ} \mathrm{C} / \mathrm{min}$. for a 40 -second period. A lower rate might be used if a transition is particularly sharp or more resolution is required. Alternatively there will be circumstances when a faster underlying heating rate might be used. Generally, in current instruments, which usually use a nitrogen purge, a 60-second period with a $2^{\circ} \mathrm{C} / \mathrm{min}$. ramp is a reasonable starting point but, as in conventional DSC, the conditions will vary according to the sample and the specific information being sought. 
The choice of modulation amplitude is firstly governed by the signal-to-noise ratio. If the amplitude is too small, then it will be difficult to detect and so the signal-to-noise will degrade. A few tenths of a degree should normally be sufficient. If the amplitude is too large then this will 'smear' the transition. Consider a glass transition that is $10^{\circ} \mathrm{C}$ wide. If the modulation amplitude is also $10^{\circ} \mathrm{C}$, then, when the average temperature is $5^{\circ} \mathrm{C}$ below its onset, the modulation will already be significantly influenced by the transition. There is also the problem of linearity. If the amplitude it too large, then the response will be significantly non-linear. A check is to change the amplitude and it should be possible to find a range of values where the result remains invariant. An amplitude of a $0.5^{\circ} \mathrm{C}$ will often give satisfactory results for the kinds of applications considered in this book.

It is possible to select a programme for a rising temperature experiment such that the minimum heating rate is always positive or zero (this is the case in Fig. 2), or the heating rate is sometimes negative. In the next section the various different types of transition that can be studied by MTDSC are discussed. In general, any type of heating programme can be used except when it is the melting behaviour that is of interest. In the case of melting, it has been shown that the material that melts while the temperature is increasing will not crystallise when the temperature is decreased $[8,10]$. This then gives rise to a highly asymmetrical and, therefore, non-linear response to the modulation. Consequently, when melting is being studied, conditions should be chosen so that the heating rate is never negative (this is sometimes referred to as "heat only" conditions)

In reality it is not possible to recommend experimental conditions that will apply very generally to a wide range of materials and types of study. The above comments are intended as a simple guide for the novice. These guidelines are often contravened in this this book! There is no substitute for gaining a good understanding of the basic theory of MTDSC and then building experience through practical study.

\subsection{COMMON TRANSFORMATIONS STUDIED BY MTDSC}

In the next part of this chapter we will consider the most commonly encountered types of processes that are studied by MTDSC in polymeric materials. The types of results they give and the appropriate specific kinetic functions will be discussed. The categories are as follows:

1) Chemical reactions and related processes.

2) Glass transitions.

3) Melting.

\subsection{CHEMICAL REACTIONS AND RELATED PROCESSES}




\subsubsection{Characteristics of MTDSC Results for Chemical Reactions and Related Processes}

In this section, the discussion will begin the simplest case that can realistically be considered a zero-order irreversible chemical reaction. In this example, the reaction rate is a function only of temperature until all reactant is consumed and the reaction stops. The exact function governing the temperature dependence of the reaction rate is not defined in this initial analysis, but it can be, it is assumed, approximated to be linear over the small temperature interval of the modulation. The more general case where the chemical reaction can be considered to be a function of time (and therefore conversion) and temperature is then treated. Finally, the Arrhenius equation is dealt with as this is the most relevant case to the subject of this book.

In the case of a zero-order reaction, the rate of the reaction is dependent only on the temperature thus it produces heat at a rate given by some function of temperature. Taking the heating programme given above:

$d Q / d t=C_{p}(\beta+\omega B \cos \omega t)-h\left(T_{0}+\beta t+b \sin \omega t\right)$

where $h(T)=$ some function that determines how the heat output from the reaction changes with temperature.

Note that the contribution to the heat flow from the sample's heat capacity is included. As discussed above, the heat capacity can be considered as the energy contained in the various vibrational, transitional etc. modes available to the sample. In this section, these processes are considered to be very fast and can normally be treated as instantaneous when compared to the frequency of the modulation that typically has a period of several tens of seconds. This means that any heat flow deriving from the heat capacity will not depend on the heating rate or frequency of the modulation. The energy contained in these molecular motions is stored reversibly. This can be contrasted with the enthalpy associated with the zero-order chemical reaction being considered in this case, which is irreversible.

It can be shown (see the section on detailed MTDSC theory) that to a good approximation under realistic conditions:

$d Q / d t=C_{p} \beta-h\left(T_{0}+\beta t\right)+\omega \mathrm{B} C_{p} \cos \omega t+\mathrm{C} \sin \omega t$

for clarity this can be rewritten as:

$$
\begin{aligned}
d Q / d t= & C_{p} \beta-h\left(T_{0}+\beta t\right) & & \ldots \text { the underlying signal } \\
& +\omega \mathrm{B} C_{p} \cos \omega t+C \sin \omega t & & \ldots \text { the response to the modulation }
\end{aligned}
$$

where $C=B d h\left(T_{0}+\beta t\right) / d \mathrm{~T}=$ the derivative of $h\left(T_{0}+\beta t\right)$ with respect to temperature. 
Note that the underlying signal is the same as would be obtained in a conventional nonmodulated experiment. Averaging over the period of a modulation will suppress the modulation. Thus,

$<d Q / d t>=C_{p} \beta-h\left(T_{0}+\beta t\right)$ 
Also:
$C_{p P C R}=C_{p}$
$C_{p K}=\left(d h\left(T_{0}+\beta t\right) / d \mathrm{~T}\right) / \omega$

Thus, it follows that

$\left\langle d Q / d t>-C_{P P C R} \beta=h\left(T_{0}+\beta t\right)=\right.$ the phase corrected non-reversing heat flow

In other words it is possible to separate the contribution in the total heat flow from the heat capacity and that which arises from the zero order reaction. It is this ability that is one of the main advantages of MTDSC. In most cases, it is not necessary to use the phase lag correction in order to achieve this, so the simple deconvolution procedure is adequate.

The above is intuitively satisfactory when one considers that, in a zero order reaction, the reaction rate will change only with temperature and will thus follow the $B \sin \omega t$ of the modulation. The contribution from the heat capacity, on the other hand, follows the derivative of temperature and thus follows $\omega B \cos \omega t$. The in-phase contribution arises from a signal that depends only on the heat capacity. Thus, this provides a means of separating or deconvoluting these two different contributions to the heat flow.

We now consider a more general process that gives rise to a heat flow and is governed by a kinetic function that is dependent on temperature and time, $f(t, T)$. The derivation of this result given below is provided in the section in this chapter on advanced theory. In effect we come to essentially the same conclusion as for the zero order case.

$$
\begin{aligned}
d Q / d t= & \beta C_{p}+f\left(t, T_{0}+\beta\right) & \ldots . . \text { the underlying signal } \\
& +\omega \mathrm{B} C_{p} \cos \omega t+C \sin \omega t & \ldots . . \text { the response to the modulation }
\end{aligned}
$$

where $C=B \frac{\partial f}{\partial T}$ (as defined above) to some approximation but may be considered to include other terms depending on the experimental conditions and the nature of the $f(t, T)$ term (see the section on detailed theory).

By analogy with the case considered above,

$<d Q / d t>-C_{p P C R} \beta=f\left(t, T_{0}+\beta t\right)=$ the phase corrected non-reversing heat flow

Thus, as also demonstrated in equation 32 , by carrying out this deconvolution procedure it is possible to separate the two fundamentally different contributions to the total heat flow: the reversible contribution that derives from the heat capacity (the phase- 
corrected reversing heat flow) and the contribution that derives from $f(t, T)$ which is, on the time scale of the modulation, irreversible. In most cases the phase-corrected reversing heat flow will be the same as the reversing heat flow to an accuracy greater than that of the measurement being made.

In the description given above, essentially represented in equations 26 to 34, the 'reversing' signal was truly reversible and the 'non-reversing' signal came from a nominally irreversible process. However the non-reversing signal can also be the heat from a crystallisation or from the loss of volatile material. Both of these processes are reversible in the sense that, with largescale temperature changes, crystals can be melted and, on cooling, moisture can be reabsorbed. For this reason the term non-reversing was coined to denote that at the time and temperature the measurement was made the process was not reversing although it might be reversible.

Most of the transitions being considered in this section will follow, to some approximation, the Arrhenius equation. Viz:

$$
d x / d t=f(x) A \mathrm{e}^{-E / R T}
$$

$$
\begin{array}{ll}
\text { where } & x=\text { the "extent" of the reaction, } \\
t= & \text { time, } \\
f(x)= & \text { some function of extent of reaction, } \\
A= & \text { the pre-exponential constant, } \\
E= & \text { the activation energy, } \\
\mathrm{R}= & \text { the gas constant, } \\
T= & \text { absolute temperature. }
\end{array}
$$

This type of behaviour is associated with the well-known energy barrier model for thermally activated processes. In this model, a material changes from one form to another more thermodynamically stable form, but must first overcome an energy barrier that requires an increase in Gibbs free energy. Only a certain fraction of the population of reactant molecules have sufficient energy to do this and the extent of this fraction and the total number of reactant molecules determine the speed at which the transformation occurs. The fraction of molecules with sufficient energy is dependent upon the temperature in a way given by the form of the Arrhenius equation. Thus, this must also be true for the transformation rate. The types of process that can be modelled using this type of expression include chemical reactions, diffusion controlled processes such as the desorption of a vapour from a solid and some phase changes such as crystallisation. There will be some constant of proportionality, $H$, such that the rate of heat flow can be directly related to the rate of the process. Viz.:

$$
(d Q / d t)_{r}=H d x / d t=H f(x) A \mathrm{e}^{-E / R T}
$$


One can derive the following equation (see the advanced theory section)

$$
\begin{aligned}
d Q / d t=\beta C_{p}+ & H f(<x>) A \mathrm{e}^{-E / R T} \\
& +B \omega C_{p} \cos \omega t+C \sin \omega t
\end{aligned}
$$

where $C=B f(\langle x\rangle) \cdot d\left(H A \mathrm{e}^{-E / \mathrm{R}\langle T\rangle}\right) / d T=B f(\langle x\rangle) \cdot\left(H A E / R\langle T\rangle^{2}\right) \mathrm{e}^{-E / R<T\rangle}$.

A typical form of $f(x)$ might be:

$f(x)=(1-x)^{n}$

where $n=$ the reaction order

However, there are many other possibilities that are already well established in the literature [27] - some of these are considered in detail in Chapter 2. Again, one can say:

$<d Q / d t>=\beta C_{p}+H f(<x>) A \mathrm{e}^{-E / R T}$

$\left\langle d Q / d t>-C_{p P C R} \beta=H f\left(\langle x>) A \mathrm{e}^{-E / R T}=\right.\right.$ the non-reversing signal

Thus it is possible to conclude that the non-reversing heat flow contains that part of the underlying signal that comes from the chemical reaction. In most cases, it is also true to a very good approximation that $C^{*}=C_{p P C R}$.

Thus it is not necessary to use the phase correction in order to measure the heat capacity and then calculate the non-reversing signal so the simple deconvolution can be used.

Also since $C_{p K}=\left(H A E f\left(\langle x>) / \omega R\langle T\rangle^{2}\right) \mathrm{e}^{-E / R<T>}\right.$ and a comparison with the phase corrected non-reversing signal shows that the activation energy is given by:

$E=\left(\omega R\left\langle T>^{2} C_{p K}\right) /\left(<d Q / d t>-C_{p P C R}\right)\right.$

Toda et al. have shown that equation 41 can be used to determine $\mathrm{E}$ [29].

Above, the simplest possible case (a zero order reaction) has been considered. Here the results are intuitively easy to understand. The general case, $f(t, T)$, where kinetics are a function of both time and temperature is then considered and essentially the same result is achieved. Finally, for completeness, the most commonly encountered case (the Arrhenius equation) is dealt with. In all of these examples we came to the same conclusions (mathematical details are given in the section on MTDSC theory).

Fig. 8 shows results for a curing sample. In the reversing signal a glass transition is observed during the course of the cure reaction which provides the enthalpy change that appears in the non-reversing signal. Clearly, it is not possible to obtain the same information from a 
conventional DSC experiment, which would not be able to separate these two contributions to the total heat flow. The advantages that this affords for the study of reacting systems are illustrated extensively in chapter 2 .

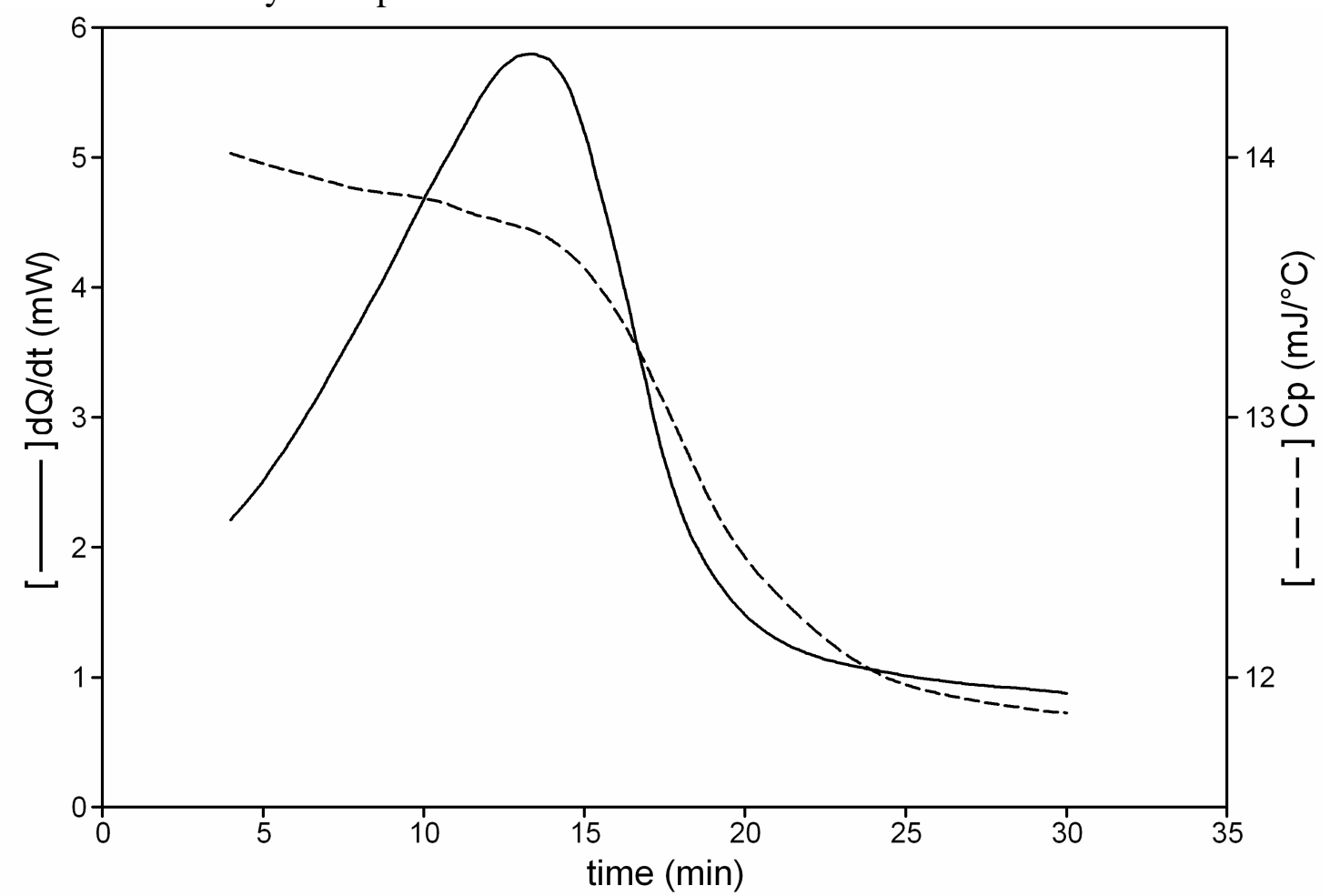

Fig. 8 Isothermal cure of an epoxy resin showing a glass transition during cure. Data from [5].

Fig. 9 shows an example of detecting a glass transition beneath a cold crystallisation exotherm. The total heat flow corresponds to conventional DSC experiment. It is not possible from inspection of the distorted peak in this curve to conclude that it is formed from an exotherm (from the crystallisation of PET) superimposed on a glass-rubber transition (from the polycarbonate). The additional signals of MTDSC make this interpretation clear. In this case, the crystallisation acts like a chemical reaction: once formed the crystals remain as the temperature increases through the peak. Thus, the process is non-reversing.

Inspection of Fig. 3 shows there is a decrease in reversing heat capacity as initially purely amorphous PET crystallises. This effect is present, but cannot be seen easily in Fig. 9 in part because the change is correspondingly smaller in this sample as there is a large amount of a second amorphous material present and also due to the increase in heat capacity through $T_{g}$. The results in Fig. 3 are an accurate reflection of the fact the crystals have a lower heat capacity than the amorphous material that proceeded them. Note also that, during the cold crystallisation, the peak in the phase lag is negative (and so, therefore, is the kinetic heat capacity). This is exactly what theoretical analysis predicts. 


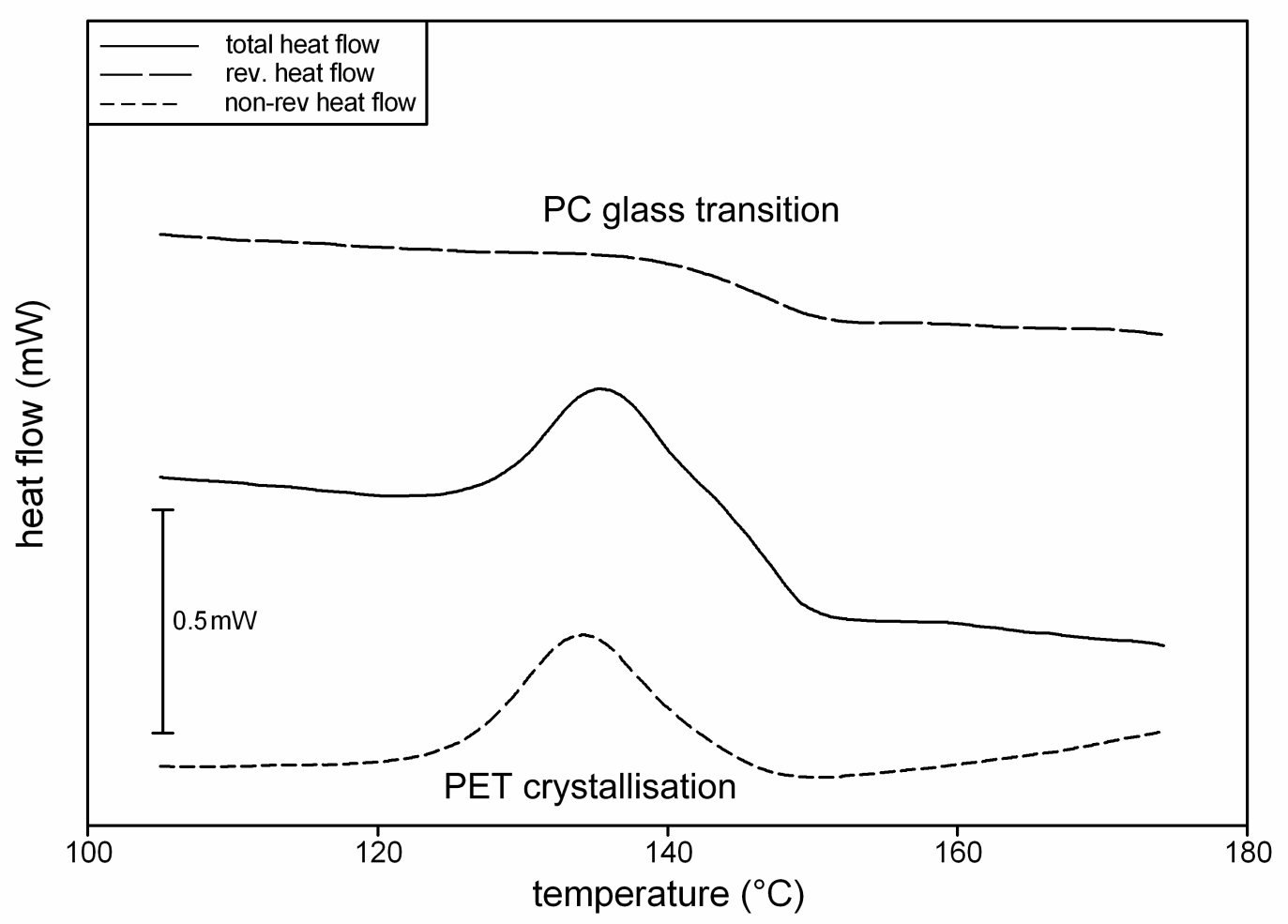

Fig. 9 Crystallisation of PET:PC bilayer film showing detection of PC glass transition during crystallisation of PET. Data from [3].

This ability to measure changes in vibrational heat capacity that occur during the course of a process that gives rise to a heat flow such as a chemical reaction or crystallisation is a very useful aspect of MTDSC. It applies equally well to the loss of volatile material, for example, that can mask a glass transition.

Often the deconvolution into reversing and non-reversing is most useful when there is a 'hidden' glass transition such as in Fig. 8 and Fig. 9. For reasons that are discussed in the section below on glass transitions, the presence of a glass transition in the reversing signal implies an error in the non-reversing signal. This is because not all of the energy changes associated with a glass transition is to be found in the reversing signal. At $T_{g}$, there is always a (usually) small non-reversing contribution. In most cases this can be neglected. Where it is important to account for this, it can be done by measuring the non-reversing signal of the relevant glass transition when other processes are not present (see [38]).

\subsubsection{Summary}

- By averaging the modulated heat flow signal one can recover results that are equivalent to conventional DSC. This is important because DSC is a highly successful technique for the good reason that the information it provides is very useful. 
- One can measure the sample's vibrational heat capacity independently of any other process that is occurring such as a chemical reaction by looking at the in-phase response to the modulation. This signal gives provides $C_{p}$ directly.

- The out-of-phase response can be expressed as the kinetic or, in complex notation, the imaginary heat capacity or simply as $C$ in many of the above equations. It can take a variety of forms depending on the details of the experiment conditions and the form of $f(t, T)$. However, it is generally approximated by taking the derivative with respect to temperature of the heat flow generated by the reaction or other process. This signal can be used to determine the activation energy for a reaction.

- Very often the out-of-phase component $C$ is small so the reversing heat capacity (modulus of the complex heat capacity) is the same as the in-phase component (phase-corrected reversing or real heat capacity) so the phase correction can be neglected. This means that the simple deconvolution defined above can be used.

- The non-reversing signal gives a measure of the energy that arises from the chemical reaction.

- Where a glass transition is present underneath a non-reversing peak due to a cure reaction or a similar transformation, then this does imply an error in the non-reversing signal because there is a non-reversing component arising from devitrification. This can usually be neglected or corrected for.

\subsection{THE GLASS TRANSITION}

\subsubsection{Characteristics of MTDSC results for Glass Transitions}

Fig. 10 shows typical MTDSC results for a glass transition for a polystyrene sample that has been annealed for different lengths of time. It can be seen that, as expected, the total signal is the same as that observed for a conventional DSC experiment. As annealing increases, the characteristic endothermic peak at the glass transition increases. At low levels of annealing there are noticeable changes in the total signal as the characteristic relaxation peak is seen to develop. However, the changes in the reversing and kinetic signals are small. It follows that the non-reversing signal shows an increasing peak with annealing time. The use of MTDSC seems to eliminate the influence of annealing and enables the relaxation endotherm to be separated from the glass transition itself. To a first approximation this is true, but this must be understood within the context of the frequency dependence of the glass transition. 


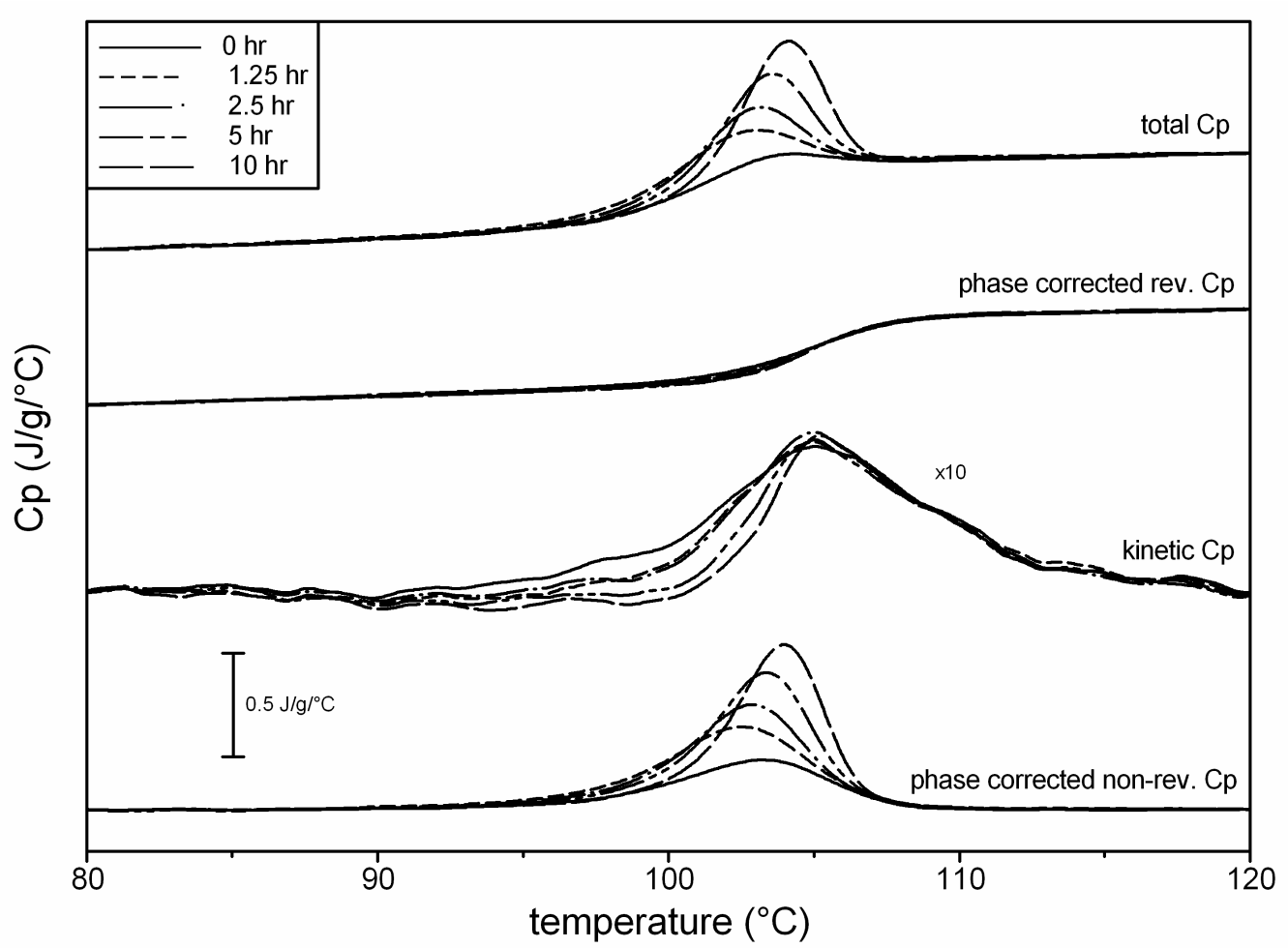

Fig. 10 Typical results for a glass transition with different degrees of annealing (polystyrene annealed at $90^{\circ} \mathrm{C}$ and re-heated at $2^{\circ} \mathrm{C} / \mathrm{min}$, period 40s, amplitude: $\mathbf{0 . 2 1 2}^{\circ} \mathrm{C}$, under helium).

It is well known that the temperature of the glass transition is frequency dependent from measurements made with dynamic mechanical and dielectric measurements. This same frequency dependence is seen in MTDSC [30]. Fig. 11 shows the results for polystyrene at a variety of frequencies. For a cooling experiment with MTDSC, there is both a cooling rate, $\beta$, and a frequency (the frequency of the modulation, $\omega$ ). If the cooling rate is kept the same and the frequency is varied, the underlying signal remains constant while the reversing signal changes. The underlying signal will always give a lower $T_{g}$ than the reversing signal because the underlying measurement must, in some sense, be slower (i.e. on a longer time scale) than the reversing measurement. This is because of the requirement that there be many modulations over the course of the transition. As the cooling rates become slower, in other words as the time scale of the measurement becomes longer, $T_{g}$ moves to a lower temperature. Similarly, as the frequency decreases, $T_{g}$ moves to lower temperatures. As a consequence of this, there is a peak in the non-reversing signal as the sample is cooled that is clearly not related to annealing, but is a consequence of the difference in effective frequency between the average measurement and that of the modulation. Thus the non-reversing signal changes with cooling rate and modulation frequency. This is shown in Fig. 11. 


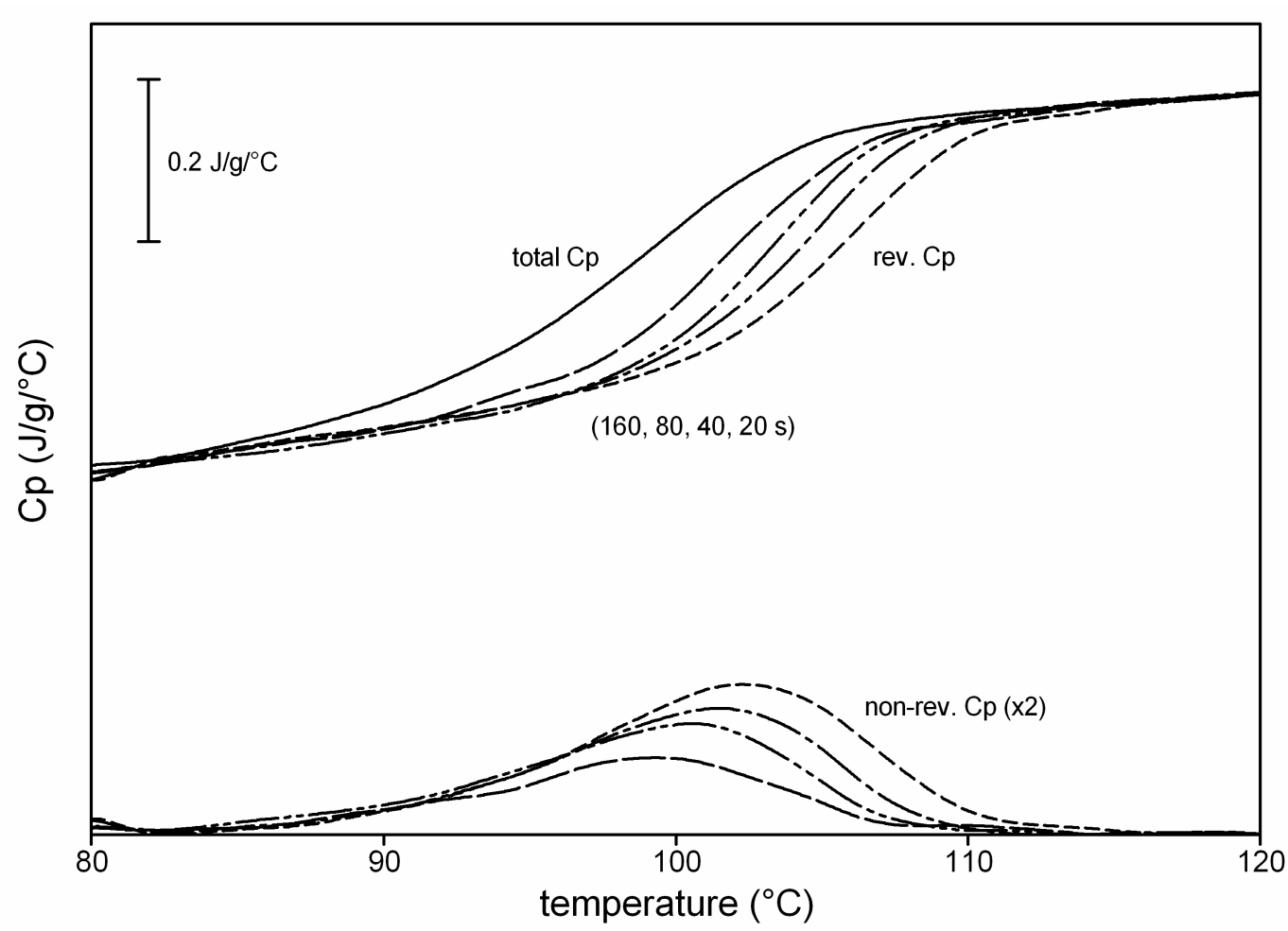

Fig. 11 Experimental results that illustrate the effect of frequency on the total, reversing and non-reversing $C_{p}$ for the glass transition of polystyrene in cooling (period 20, 40, 80 and $160 \mathrm{~s}$, underlying rate: $1^{\circ} \mathrm{C} / \mathrm{min}$, 'cool only', under helium).

On heating, the non-reversing signal, as can be seen from Fig. 10, is related to the amount of annealing and also must contain the effects of the different effective frequencies used in the measurement. These effects can be treated as additive. Thus, the non-reversing signal gives a measure of the enthalpy loss on annealing with an offset due to the frequency difference. This is intuitively satisfactory, as the enthalpy that is regained by the sample on heating after annealing cannot be lost again on a short time scale at the time and temperature the measurement is made. In this sense it is non-reversing in the same way that a chemical reaction or crystallisation is. This simple picture is only a first approximation, but it will be adequate in many cases. In particular the non-reversing peak at the glass transition can be used to rank systems in terms of degree of annealing. 


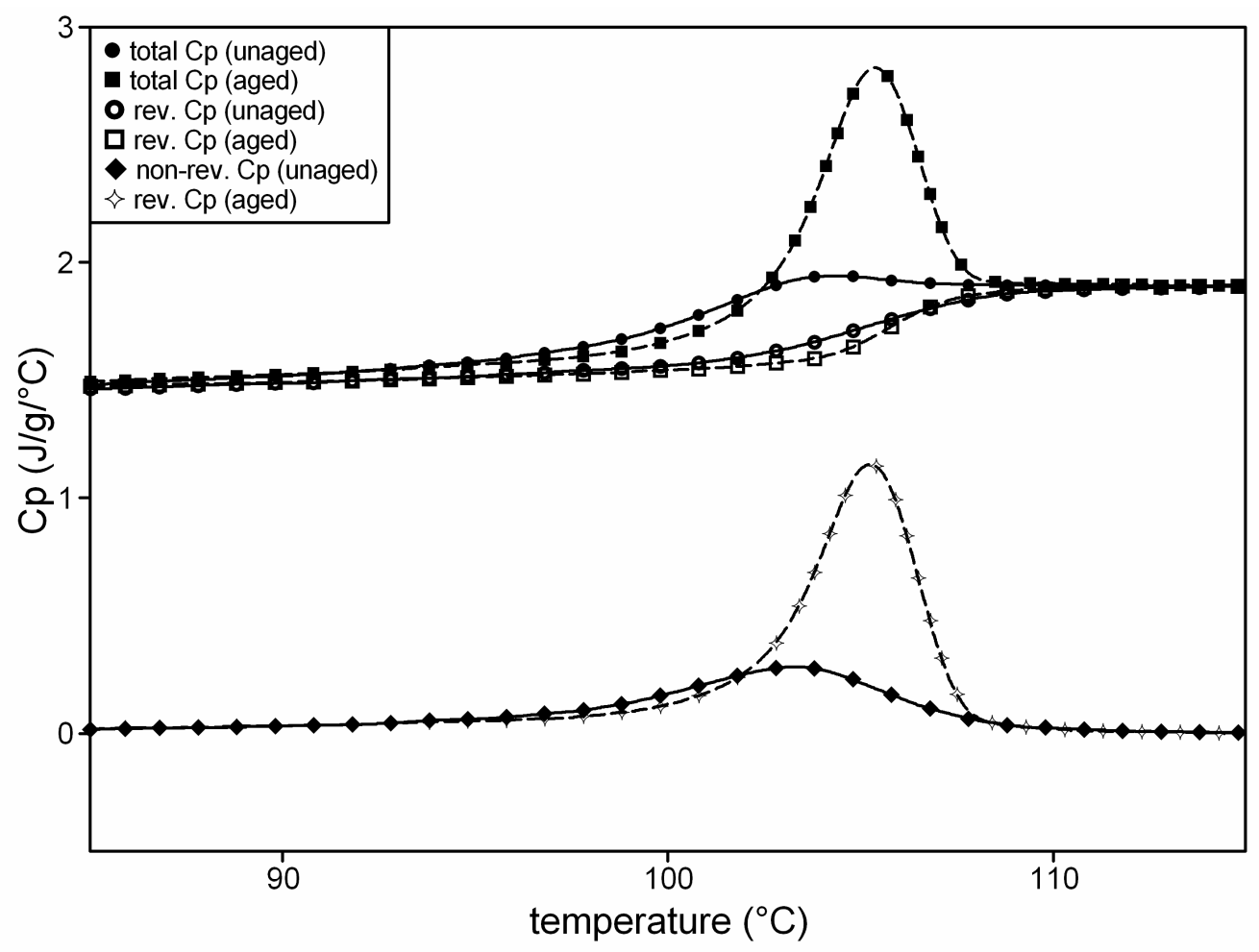

Fig. 12 Effect of long annealing times on the total and reversing signals (polystyrene unaged and aged at $90^{\circ} \mathrm{C}$ for $40 \mathrm{~h}$, reheated at $2^{\circ} \mathrm{C} / \mathrm{min}, 40 \mathrm{~s}$ period, amplitude: $0.212^{\circ} \mathrm{C}$, under helium).

In the discussion above it is assumed that, as indicated in Fig. 10, the reversing signal is not affected by annealing. In reality this is not correct. At higher degrees of annealing, the reversing signal becomes sharper thus the simple relationships outlined in the previous paragraph break down. This is illustrated in Fig. 12, which compares the behaviour of a sample of polystyrene that has been subjected to a low and a high level of annealing. Fig. 13 shows that slower cooling rates also lead to sharper reversing transitions. In both cases the sample is closer to equilibrium when it undergoes the transition in the reversing signal and this leads to a narrowing of the temperature range over which it occurs. How this can be allowed for is discussed below.

At first sight, the step change in $C_{p}$ that occurs at the glass transition might be interpreted as a discontinuity; that would mean that it would be a second-order transition. In fact the transition is gradual as it occurs over about $10^{\circ} \mathrm{C}$ or more. Its position also varies with heating rate (and with frequency in MTDSC) which reveals that it is a kinetic phenomenon. The co-operative motions that enable large-scale movement in polymers have activation energies in a way that is similar to (but not the same as) the energy barrier model mentioned above for Arrhenius processes. Thus, as the temperature is decreased, they become slower until they appear frozen. There is a contribution to the heat capacity that is associated with these motions. Therefore, as the temperature is reduced, these large-scale motions are no longer possible and 
consequently the material appears glassy (rigid) and the heat capacity decreases. In reality, whether a polymer appears glassy or rubbery depends the timescale of the observation. Thus, if the polymer is being vibrated at a frequency of several times a minute, it may be springy and return to its original shape when the stress is removed. If it is being deformed and released over a period of a year, it may well behave like a pliable material that creeps under load, thus retaining a permanent distortion in dimensions when unloaded. There is a parallel dependence of the heat capacity on how rapidly one is attempting to put heat into or take it out of the sample, thus the position of $T_{g}$ changes with heating and cooling rates.

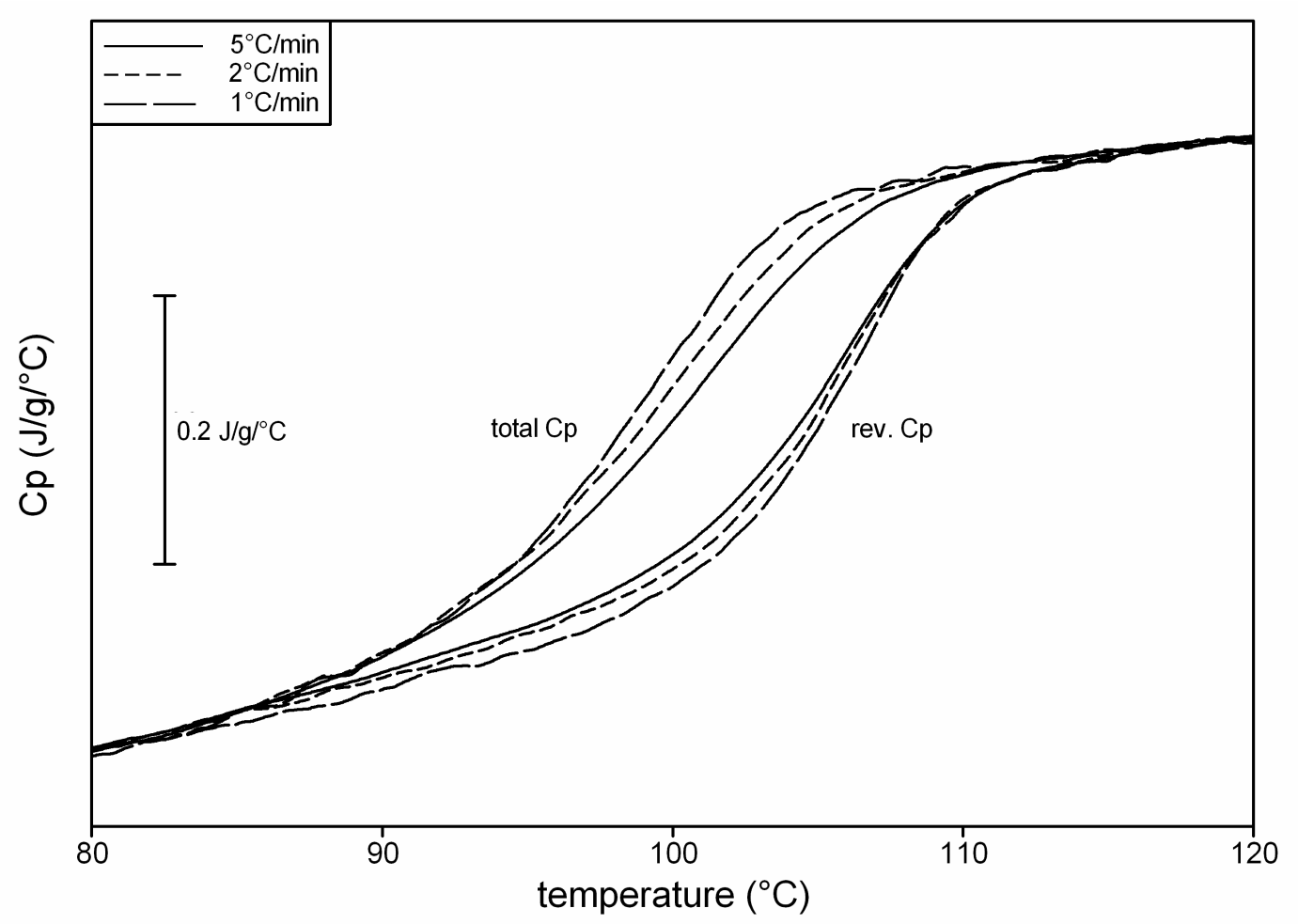

Fig. 13 Experimental results that illustrate the effect of cooling rate on the total and reversing $C_{p}$ for the glass transition of polystyrene (period $20 \mathrm{~s}$, underlying rate: 1 , 2 and $5^{\circ} \mathrm{C} / \mathrm{min}$, 'cool only', under helium).

Figs. 14 and 15 give the enthalpy and heat capacity diagrams for glass formation. The enthalpy gained, or lost, by a sample is determined by integrating the area under the heat capacity curve. Above $T_{g}$ the sample is in equilibrium (provided no other processes such as crystallisation are occurring). Consequently, this line is fixed regardless of the thermal treatment of the sample and a given temperature corresponds to a unique enthalpy stored within the sample. As the sample is cooled, there comes a point at which the $C_{p}$ changes as it goes through the glass transition. Thus, $d Q / d t$ changes and so does the slope of the enthalpy line. At different cooling rates, the temperature at which this occurs changes. Thus a different glass with a different enthalpy is created. Above the transition, the sample is at equilibrium. Below 
$T_{g}$, it is at some distance from this equilibrium line, but is moving towards it very slowly. Thus, glasses are metastable. If the glass is annealed at temperatures a little below the glass transition, it looses enthalpy relatively rapidly and becomes a different glass as it moves toward the equilibrium line. At temperatures far below $T_{g}$, the rate of enthalpy loss becomes very slow and effectively falls to zero. When the sample is heated, the enthalpy lost on annealing must be regained and this gives rise to the characteristic peak at the glass transition seen in Fig 10.

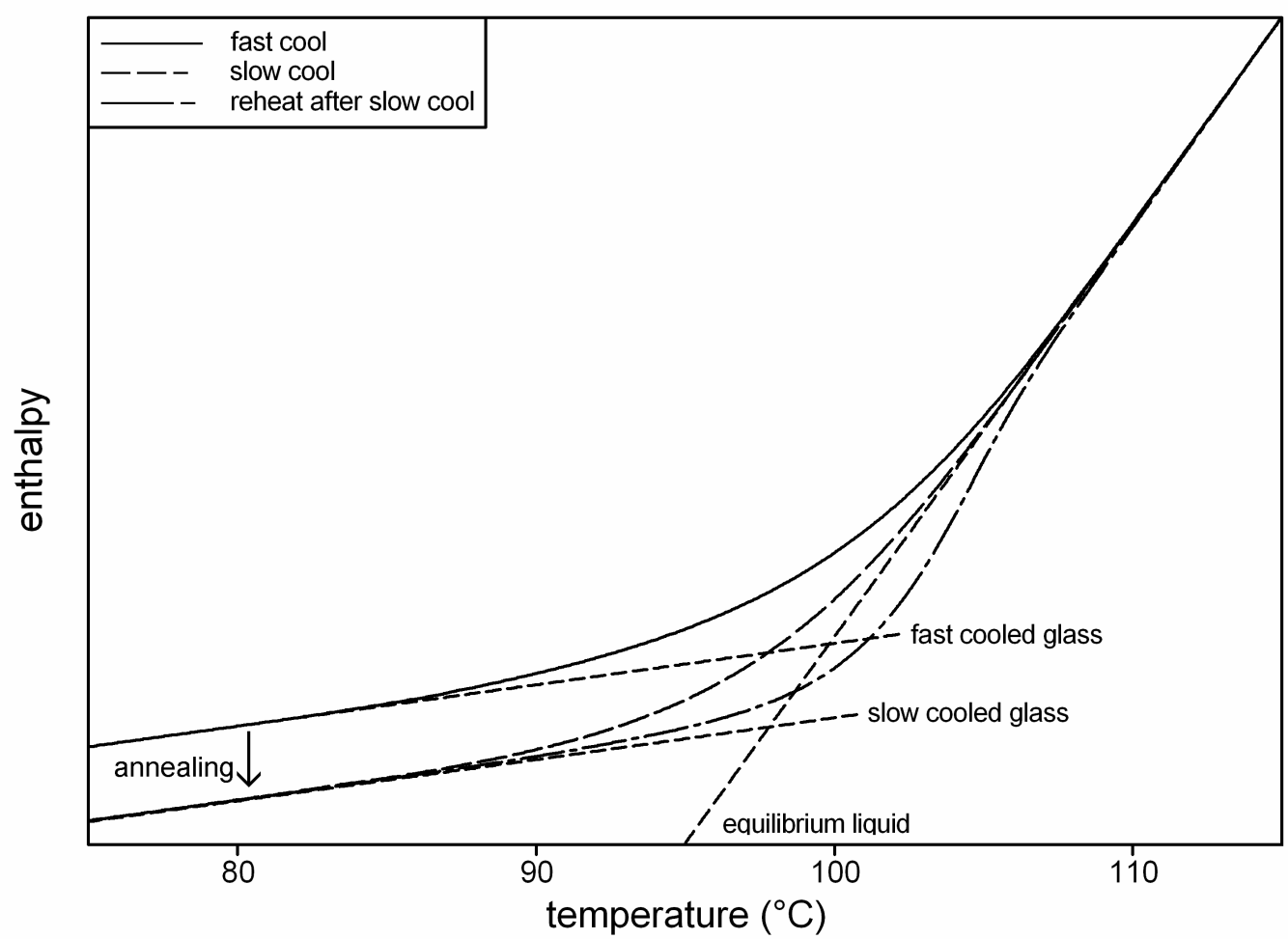

Fig. 14 Enthalpy diagrams for the glass transition - original data obtained on polystyrene, the change in $C_{p}$ between the glass and rubber has been exaggerated for clarity.

From a simple model [31] of the glass transition it is possible to derive approximate analytical expressions that model the response to the modulation at the glass transition (see the discussion in Section 4.4). Viz:

$$
\begin{aligned}
& \Delta C_{p P C R}=\Delta C_{\mathrm{p}} /\left(1+\exp \left(-2 Q_{\omega} \Delta h^{*}\left(T-T_{\mathrm{g} \omega}\right) / \mathrm{R} T_{g \omega}{ }^{2}\right)\right) \\
& C_{p K}=q \Delta C_{p P C R} \exp \left(-Q_{\omega} \Delta \mathrm{h}^{*}\left(T-T_{\mathrm{g} \omega}\right) / R T_{g \omega}{ }^{2}\right) /\left(1+\exp \left(-2 Q_{\omega} \Delta h^{*}\left(T-T_{g \omega}\right) / R T_{\mathrm{g} \omega}{ }^{2}\right)\right)
\end{aligned}
$$

where $\Delta C_{p}=$ the change in heat capacity at the glass transition,

$Q_{\omega} \& q=$ shape factors related to the distribution of relaxation times and mechanism of the relaxation process 
$\Delta h^{*}=$ the apparent activation energy

$T_{g \omega}=$ the glass transition temperature (at half-height) at frequency $\omega$.

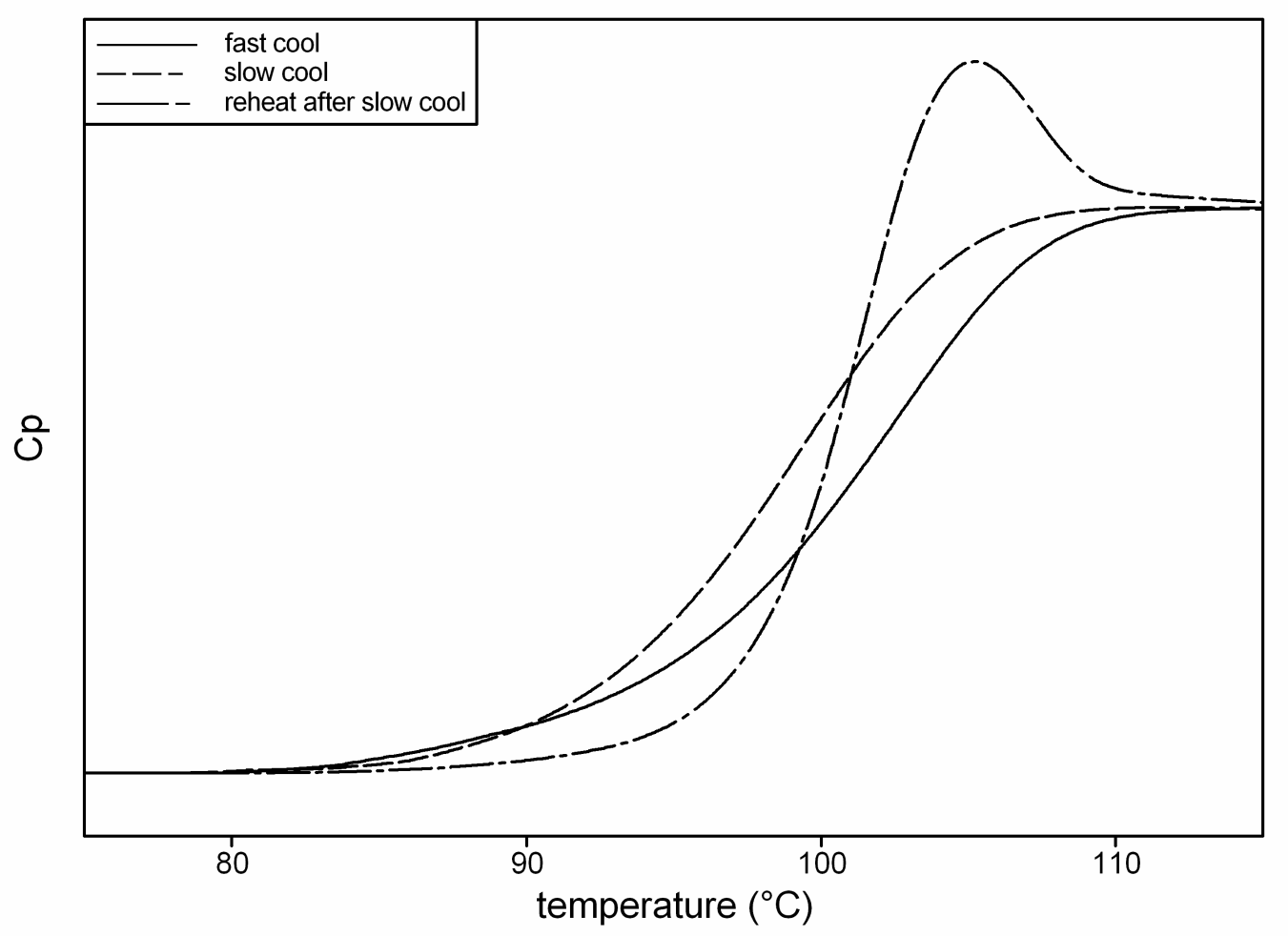

Fig. 15 Schematic heat capacity plot that corresponds to the enthalpy diagram shown in Fig. 14 showing the peak in $C_{p}$ arising from annealing.

The $T_{g \omega}$ is given by relating the period of oscillation to the timescale associated with the Arrhenius relationship. Viz:

$$
\omega=A \mathrm{e}^{-\mathrm{Ah} * / \mathrm{R} T g \omega}
$$

The change in the average or total signal for heating or cooling rate $\beta$ can be approximately modelled the following relationship:

$$
\Delta C_{p T \beta}=\frac{\Delta C_{p}}{\left(1+\exp \left(-2 Q_{\beta} \Delta h^{*}\left(T-T_{g \beta}\right) / R T_{g}^{2}\right)\right)}+\frac{n_{h}}{\beta} \frac{\exp \left(T-T_{g \beta}\right)}{\left(1-\exp \left(T-T_{g \beta}\right)^{2}\right)}
$$

here $T_{g \beta}=$ the apparent glass transition temperature for the total signal

$Q_{\beta}=$ shape factor for the underlying measurement at heating/cooling rate $\beta$ where this would generally be different on heating and cooling $n_{h}=$ a measure of the extent of ageing. 
Equation 44 is an ad-hoc model that is used here for illustrative purposes. $T_{g \beta}$ would normally show an Arrhenius dependence on cooling rate:

$\beta=z A \mathrm{e}^{-\Delta h^{*} / \mathrm{R} T g \beta}$

Where $z$ is some constant with units of ${ }^{\circ} \mathrm{C}^{-1}$. Where $\mathrm{z}$ is assumed to be a constant with units of ${ }^{\circ} \mathrm{C}$-1. In fact this pre-exponential factor can be considered to be a function of heating rate but this is beyond the scope of this discussion. For any frequency, there must be a cooling rate that would give the same transition temperature (taken at the half-height of the step change) and so there should be a frequency-cooling rate equivalence. These must of course be obtained from two separate experiments as these signals can never give the same $\mathrm{T}_{\mathrm{g}}$ in a single experiment. One way of looking at this is to think in terms of the time taken to traverse the transition as (with suitable weighting) a measure of the time-scale of the linear cooling rate measurement. This then is related to the period that gives a measure of the time-scale of the cyclic measurement. Thus $\beta$ and $\omega$ can be related by

$z \omega=\beta$

The concept of a reversing response can be extended to the total signal by considering a heating and cooling experiment at the same rate. The vibrational heat capacity of a purely inert sample should be exactly the same at any temperature and so completely reversing (and reversible). An experiment where the sample is cooled through a glass transition, and then heated at the same rate, will give a similar but not identical result in both directions. Because of this, it is convenient to define a hysteresis factor $\left(h_{\beta}\right)$ describing this difference:

$h_{\beta}=\Delta C_{p T \beta \mathrm{H}}-\Delta C_{p T \beta C}$

where $\Delta C_{p T \beta C}=$ the change in heat capacity on cooling

$\Delta C_{p T \beta H}=$ the change in heat capacity on heating without annealing

It is possible to raise objections to this approach on the basis that change below $T_{g}$ never ceases thus there is no end to the transition region on cooling and so any choice of temperature at which to reverse the cooling programme is arbitrary. This implies that the shape of the heating curve cannot be fixed. However, in reality, the rate at which the sample approaches the equilibrium line decreases very rapidly below the glass transition. Thus, a few tens of degrees below the mid-point of the step change, the transition can be said to have come to an end. 
To a reasonable approximation:

$$
\begin{array}{r}
\Delta C_{p T \beta C}=\Delta C_{\mathrm{p}} /\left(1+\exp \left(-2 \mathrm{Q}_{\mathrm{C} \beta} \Delta h^{*}\left(T-T_{\mathrm{gC} \beta}\right) / \mathrm{R} T_{g C \beta}{ }^{2}\right)\right) \\
\Delta C_{p T \beta H}=\Delta C_{\mathrm{p}} /\left(1+\exp \left(-2 \mathrm{Q}_{\mathrm{H} \beta} \Delta h^{*}\left(T-T_{\mathrm{gH} \beta}\right) / \mathrm{R} T_{g C \beta}{ }^{2}\right)\right)+ \\
\mathrm{n}_{\mathrm{h}}\left(\exp \left(\mathrm{T}-\mathrm{T}_{\mathrm{m}}\right) /\left(1-\exp \left(\mathrm{T}-\mathrm{T}_{\mathrm{m}}\right)^{2}\right) / \beta\right.
\end{array}
$$

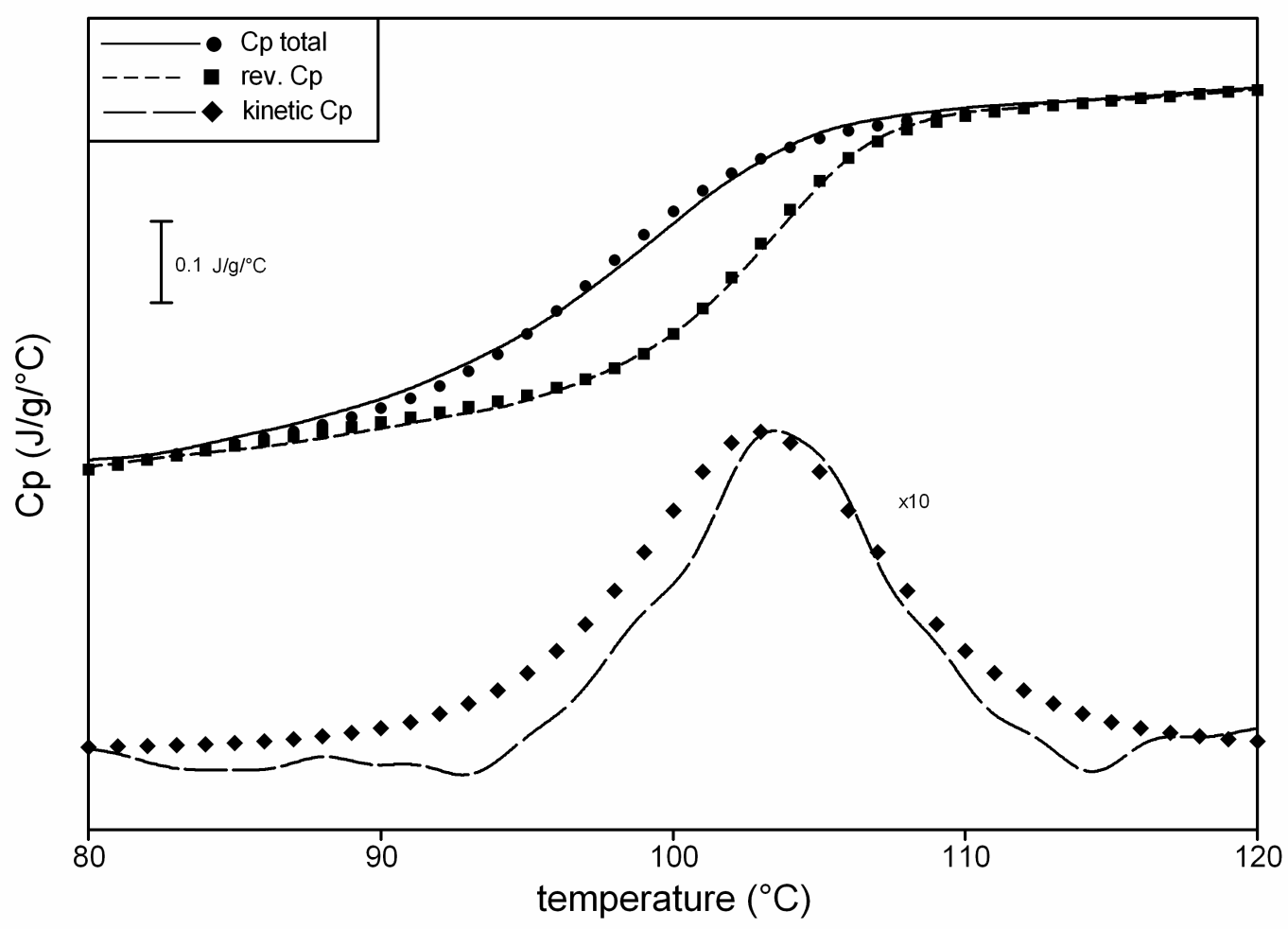

Fig. 16 Results of modelling the glass transition behaviour of polystyrene in cooling at $80 \mathrm{~s}$ period shown in Fig. 11 by applying equations 41,42 and $44\left(\Delta h^{*}=690\right.$ $\left.\mathrm{kJ} / \mathrm{mol}, T_{g \omega}=105.5^{\circ} \mathrm{C}, Q_{\beta}=0.3, T_{\mathrm{g} \beta}=93^{\circ} \mathrm{C}, Q_{\omega}=0.45, q \Delta C_{p}=0.0078 \mathrm{~J} /{ }^{\circ} \mathrm{C} / \mathrm{mol}\right)$

It is also convenient to define a function for the enthalpy recovery at $T_{g}$ due to any annealing:

$N(t, T)=n_{a}\left(\ln \left(1+1 / \exp \left(T-T_{p}\right) \cdot \exp \left(T-T_{p}\right)\right) /\left(1+\exp \left(T-T_{p}\right)\right)\right.$

so that:

$\int N(t, T)=$ enthalpy loss on annealing

Combining these equations we obtain: 
$\Delta C_{p T \beta}=\Delta C_{p} /\left(1+\exp \left(-2 Q_{C \beta} \Delta h^{*}\left(T-T_{\mathrm{gC} \beta}\right) / \mathrm{R} T_{g C \beta}{ }^{2}\right)\right)+h_{\beta}+N(t, T) / \beta$

where $h_{\beta}$ and $N(t, T)$ are both zero on cooling and $N(t, T)$ is zero on heating after cooling with no annealing.

Note that equations $41,42,44,47-49$ and 51 give the behaviour of the step change at the glass transition. For a more complete model, the change in heat capacity as a function of temperature must be taken into account above and below $T_{g}$. To deal with this, all equations that feature $\Delta C_{\mathrm{p}}$ can be adapted to follow real world behaviour by assuming a linear function above and below the glass transition. Taking equation 41 as an example, yields:

$$
C_{p P C R}=\left(\left(a_{2}-a_{1}\right) T+\left(b_{2}-b_{1}\right)\right) /\left(1+\exp \left(-2 \mathrm{Q}_{\omega} \Delta h^{*}\left(T-T_{g \omega}\right) / \mathrm{R} T_{g \omega}{ }^{2}\right)\right)+\left(a_{1} T+b_{1}\right)
$$

where $C_{p g}=a_{1} T+b_{1}$ below $T_{g}, a_{1}$ and $b_{1}$ being constants and $C_{p l}=a_{2} T+b_{2}$ above $T_{g}, a_{2}$ and $b_{2}$ being constants

This modification can also be applied to equations 42, 44, 47-49 and 51. Fig. 16 provides an example of fitting with this expressions for all of the signals on cooling while Fig. 17 gives examples of fitting to heating curves with different degrees of annealing.

If we return to the general expression for heat flow for MTDSC we can express the response at the glass transition as follows:

$$
\begin{aligned}
d Q / d t=\beta C_{p C \beta} & +\langle f(t, T)> & \ldots \text { the underlying signal } \\
& +C_{p \omega} B \omega \cos \omega t+C \sin \omega t & \ldots . \text { the response to the modulation }
\end{aligned}
$$

where $C_{p \omega}=$ the heat capacity at the frequency $\omega$, given approximately by Equation

41 , the transition temperature and shape factor changes slightly with high levels of annealing or very slow cooling.

$C_{p \subset \beta}=$ the 'reversing' heat capacity implied by the heating or cooling rate $\beta$, given approximately by Equation 51 .

$C=$ the kinetic response $=B \omega C_{p K}$ where $C_{p K}$ is given by equation 42 , this signal becomes higher and narrower and occurs at a higher temperature with high levels of annealing.

$\langle f(t, T)\rangle=h_{\beta}+N(t, T) / \beta$ This expresses changes below $T_{g}$ that give rise to the hysteresis when heating with no annealing plus the enthalpy recover at $T_{g}$ caused by any annealing. This can be approximately modelled by equations 47 and 50 . 


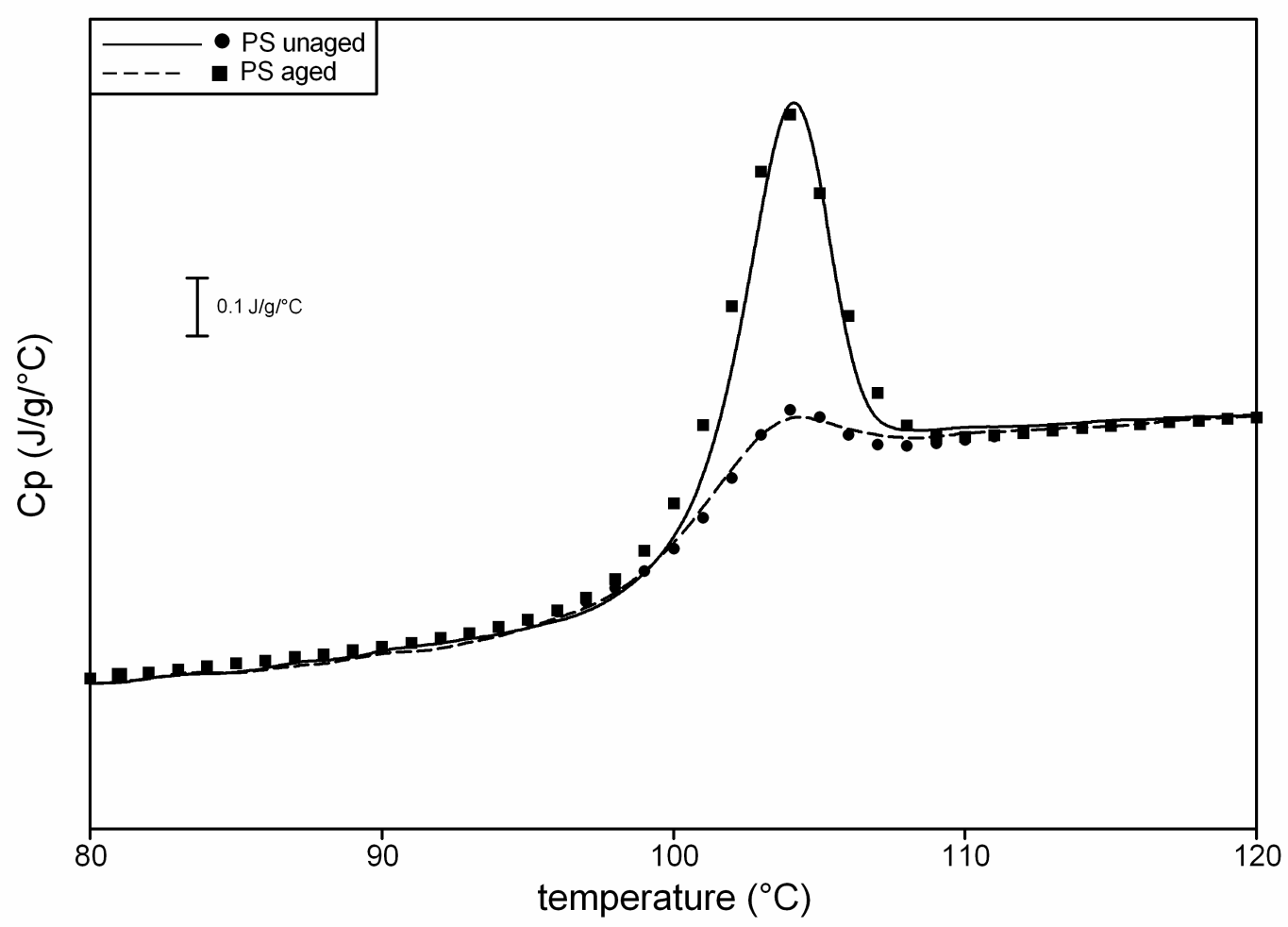

Fig. 17 Results of modelling the glass transition behaviour (total signal) of polystyrene unaged and aged for $10 \mathrm{~h}$ at $90^{\circ} \mathrm{C}$ in figure 8 by applying equation $51\left(T_{g \beta}=373 \mathrm{~K}, Q_{\beta}\right.$ $=0.3, n=0.4($ unaged $), 1.8($ aged $))$

The value of the approximate analytical expressions given in equations 41-52 is that they enable the experimenter to gain an intuitive understanding of the phenomenology of the glass transition simply by inspection. The form of $C_{p K}$ in Equation 29 may be very different from the case of a chemical reaction as given in equations 28, 33 and 37 . However, it is still basically a manifestation of the kinetics of the glass transition. Thus, the concept that this sgnal is a measure of the kinetics of the transition, remains valid. An exact description of the nonreversing signal at $T_{g}$ is complex because of the influence of the time-scale dependence of all measurements at the glass transition. However, for a sample cooled at a certain rate, annealed then heated at the same rate, the non-reversing signal contains the enthalpy recovery necessitated by the annealing. Although reversible on a sufficiently long time scale, the enthalpy recovery due to annealing is non-reversing under the conditions of the measurement. In this way, it is similar to the non-reversing signal obtained during, for example, a reversible chemical reaction. In the discussion on advanced theory models are discussed that are also phenomenological but they have fewer variables and provide for a more fundamental insight into the underlying mechanisms governing the glass transition. However, they have to be solved numerically and thus cannot by simple inspection provide a guide to thought. The model expressed in equations $41-52$ is in part based on these models but it is principally designed as 
an aid to understanding the behaviour (rather than its causes). A detailed discussion of the fundamental nature of the glass transition is beyond the scope of this chapter.

MTDSC has several significant practical advantages for studying glass transitions. The first is that the limit of detection is increased. The effect of using a Fourier analysis to eliminate all responses not at the driving frequency of the modulation reduces unwanted noise. The second is that it increases resolution. A high signal from the heat capacity is assured by a high rate of temperature change over the course of a modulation: a high resolution can be assured by using a low underlying rate of temperature increase. The third is that it makes the correct assignment more certain. When a glass transition is weak, and set against a rising baseline due to the gradual increase in heat capacity of other components, the presence of a relaxation endotherm can give the impression of a melt or some other endothermic process rather than a glass transition. A clear step change in the reversing signal makes a correct assignment unequivocal in most cases. A fourth advantage is that quantification of amorphous phases is made more accurate. The increase in signal to noise already discussed above is obviously helpful in this respect. In addition, the suppression of annealing effects makes it easier to quantify the increase in heat capacity correctly. Examining the derivative of the reversing heat capacity with respect to temperature is the best approach to doing this. This approximates very well to a Gaussian distribution and numerical fitting procedures can be used to quantify multiple phases. This is explored in detail in Chapter 3 on polymer blends.

\subsubsection{The Fictive Temperature and Enthalpy Loss on Annealing}

The fictive temperature $\left(T_{g f}\right)$ can be obtained by extrapolation of the linear portions of the enthalpy lines above and below the glass transition as illustrated in Fig. 18. This can be calculated in the case of MTDSC from the following approximate relationship:

$T_{g f}=T_{g r}+\Delta H_{N R} / \Delta C_{p}$

where $T_{g f}=$ the fictive temperature

$T_{g r}=$ the glass transition at the mid-point of the reversing signal

$\Delta H_{N R}=$ the area under the non-reversing curve (i.e. the area between the reversing and non-reversing curves)

$\Delta C_{p}=$ the heat capacity change at the glass transition 


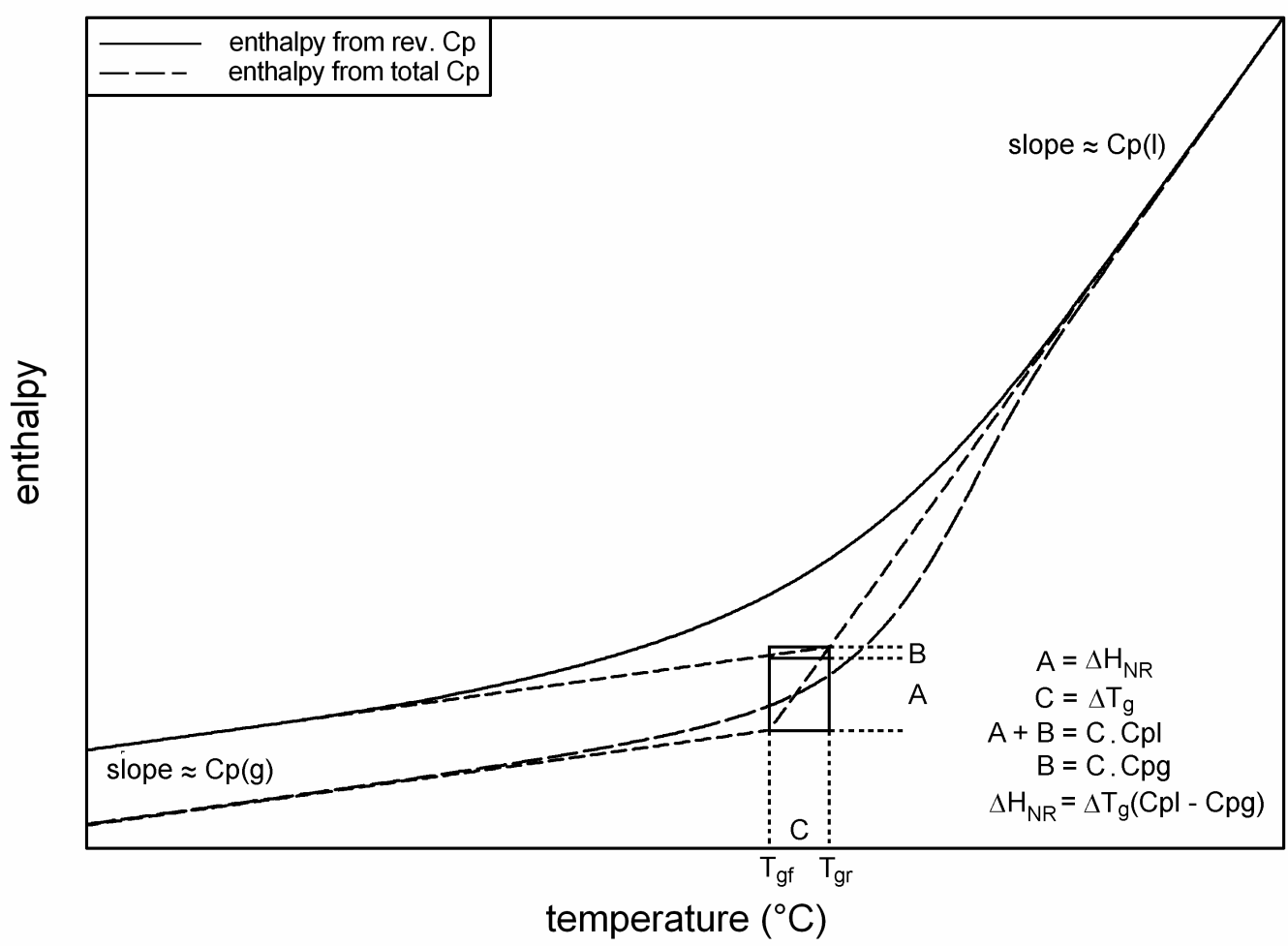

Fig. 18 Schematic diagram illustrating the relationships between fictive temperatures, enthalpy and heat capacity.

The geometric relations illustrating this equation are given in Fig. 18. If enthalpies are required relative to some reference glass, then one approach is to use the following equation:

$$
\Delta H=\left(T_{g f r^{-}} T_{g f m}\right) \Delta C_{p}
$$

where $\Delta H=$ difference in enthalpy between reference state and the measured sample

$T_{g f r}=$ fictive temperature of the reference state

$T_{g f m}=$ fictive temperature of the measured sample

Equations 54 and 55 can be criticised because they assume a unique value for $\Delta C_{p}$ whereas this varies slightly as the liquid and glass heat capacities have different slopes. (For highest accuracy $\Delta C_{p}$ should be determined for the mean of $T_{g f}$ and $T_{g r}$ ). Alternatively, enthalpy loss on annealing is often measured by using a result from a sample with low annealing (say cooled at a specified rate then immediately heated again at that rate) as the baseline that is subtracted from an annealed sample. At low degrees of annealing there should be an approximately linear relationship between this measurement and the area under the non-reversing signal because the reversing signal is not greatly affected by low small amounts of annealing. This is illustrated in Fig. 19. 


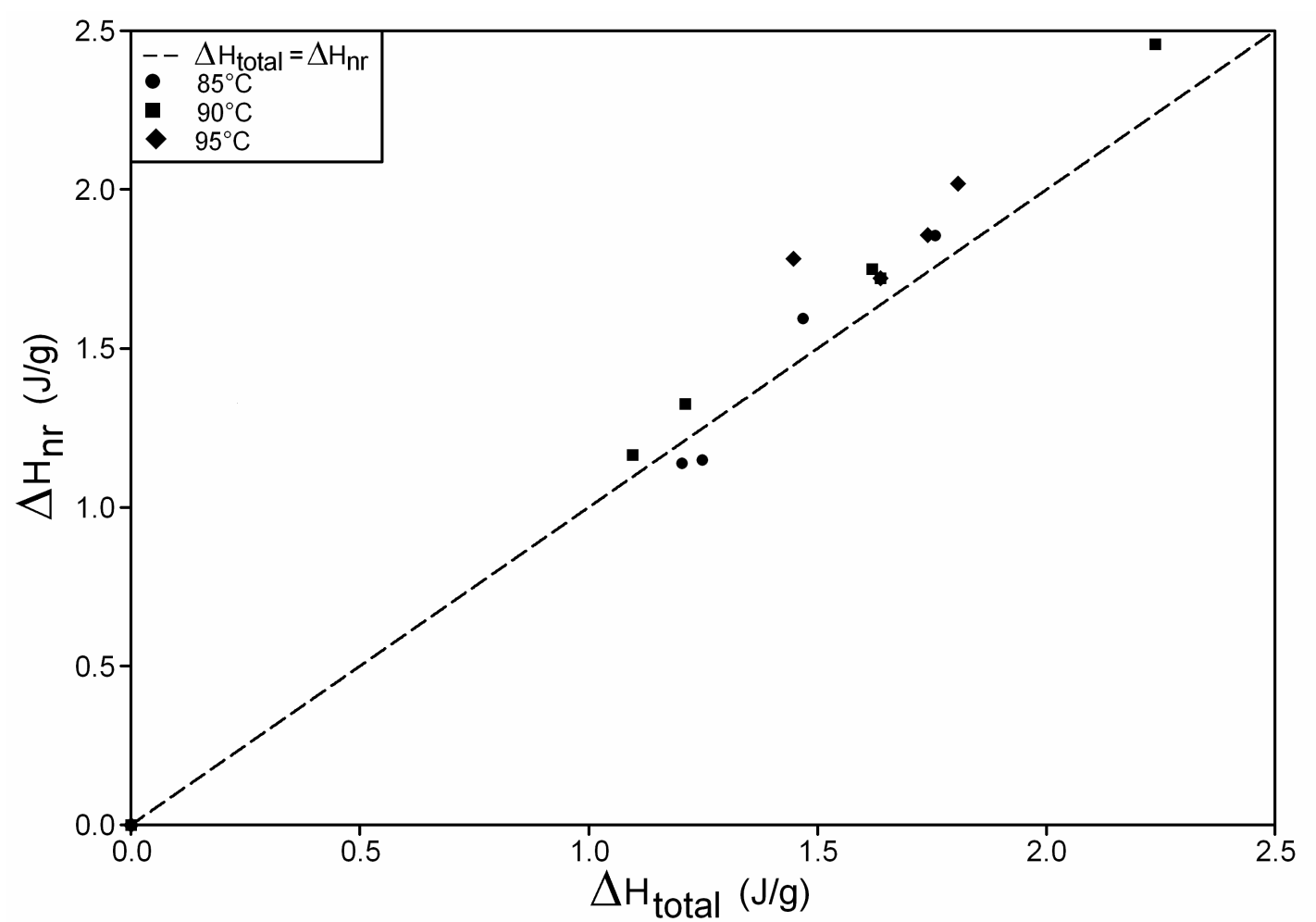

Fig. 19 Relationship between enthalpy change on ageing obtained from total $C_{p}$ and non-reversing $C_{p}$ for polystyrene aged for different times at 85,90 and $95^{\circ} \mathrm{C}$.

The early points show that the scatter in the data is greater than the deviation from the linear relationship, then there is a clear positive deviation as annealing increases, which can exceed $20 \%$ [16], as we would expect. This observation has also been made by Hutchinson [32,33] and Monserrat [34] who confirmed the earlier work of Reading et al. [16], but drew the overly pessimistic conclusion that the non-reversing signal could not be used for measuring enthalpy loss. Fig. 19 here, Fig. 4 in [34] and [35] demonstrate that, while there are problems for highly annealed samples, for low degrees of annealing a linear relationship can be assumed. In reality a deviation of the order of $5-10 \%$, which is what is found at moderate annealing, is within the scatter that would typically be expected with two different operators making ostensibly the same measurements. Experimenters must judge for themselves whether this is adequate for their needs. Certainly it is good enough to make comparisons between samples. However, any debate on this subject is redundant for two reasons. The first is that the changes in the reversing signal can easily be compensated for using the following correction:

$\Delta H=\Delta H_{N R}+\Delta T_{g r} \Delta C_{p a}$

where $\Delta T_{g r}=$ the change in the reversing glass transition temperature. 


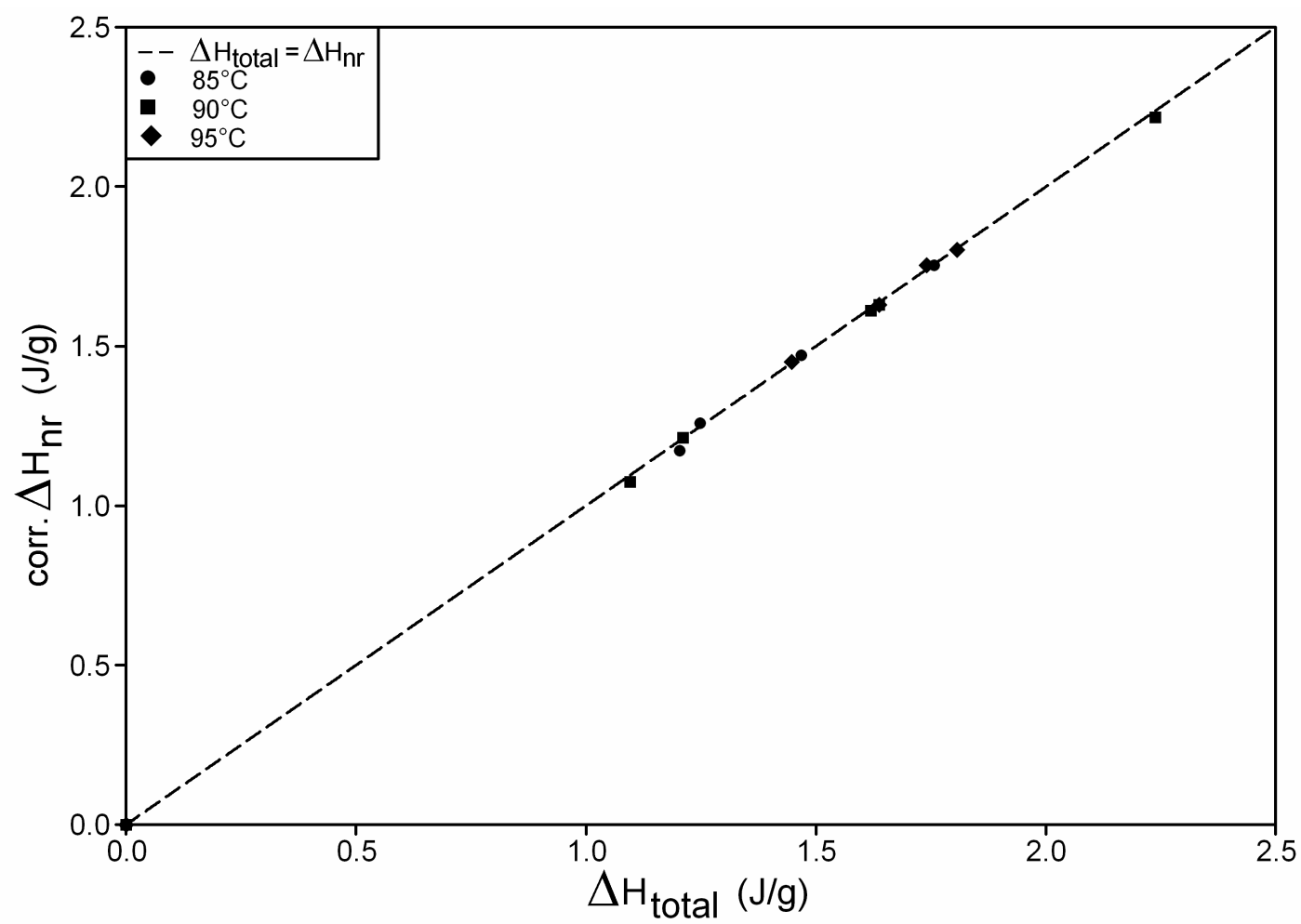

Fig. 20 As Fig. 19 following correction due to change in reversing $T_{g}$.

Fig. 20 illustrates how applying this correction excellent agreement with the more conventional approach is achieved. The second is that, whilst is useful to understand the relationships between the results given by MTDSC and the kind of parameters often determined by conventional DSC (such as fictive temperature and enthalpy loss), MTDSC does not afford any advantages over conventional DSC for such studies. Conventional DSC measurements are to be preferred in this case due to shorter measurement times and less data processing [36].

\subsubsection{Summary}

In summary, the important concepts that should be born in mind when considering glass transitions are as follows;

- The glass transition temperature $\left(T_{g}\right)$, as measured by the reversing heat capacity, is a function of frequency.

- $T_{g}$ as measured by the total heat capacity on cooling is a function of cooling rate.

- Broadly, there is equivalence between these two observations because both changing the frequency of the modulation and the cooling rate changes the time scale over which the measurement is made. This means that there is always, in the non-reversing signal, a 
contribution from $\beta\left(C_{p \beta}-C_{p \text { ? }}\right)$ which is present regardless of annealing. (For example it is present when cooling.)

- Ageing below the glass transition produces enthalpy loss that is recovered as a peak overlaid on the glass transition. However, this ageing does not, at low degrees of annealing, have a great effect on the reversing signal and this is intuitively satisfactory as the ageing effect is not reversible on the time scale of the modulation. This means that the nonreversing signal includes a contribution from the different timescales of the cyclic and underlying measurements plus a contribution from annealing expressed as $N(t, T)$ in equation 50. This implies that the relationship between the enthalpy loss on annealing and the area under the non-reversing peak should be linear.

- At high degrees of annealing, the reversing signal is affected and the non-reversing signal no longer increases linearly with enthalpy loss. However, this can be compensated for by use of the fictive temperature and associated equations such as equation 56.

- The fact that the reversing signal is largely unaffected by annealing and its derivative provides an approximately Gaussian peak makes it a much better signal for assessing the structure of blends as described in chapter 3 .

\subsection{MELTING}

\subsubsection{Characteristics of MTDSC Results for Polymer Melting}

A first-order phase transition is characterised as a change in specific volume accompanied by a latent heat. The most common example studied by DSC is melting. Typically, at the melt temperature, the sample will remain isothermal until the whole sample has melted. The factor that determines the speed of the transition is the rate at which heat can be supplied by the calorimeter. Normally this is fast compared with the overall rate of rise of temperature so the transition is very sharp with a little 'tail' the length of which is determined by the speed with which the calorimeter can re-establish the heating programme within the sample. The area under the peak is a measure of the latent heat of the transition.

Pure, low molecular weight organic materials generally produce very narrow melting peaks. Because this narrow temperature range inevitably lies, either entirely or to a significant extent, within the course of only one modulation, it means that the response to the modulation will not be linear and the deconvolution procedure we have described above cannot be used. It is possible to obtain useful information by looking at the Liassajous figures generated by the modulation. This is dealt with in chapter 4 , which covers melting.

Polymers, in contrast, produce a range of crystallites with different melting temperatures. Typically, semi-crystalline polymers will contain a distribution of crystals with differing degrees of perfection and thus different melting temperatures. The melting transition in these materials is broad, as a succession of crystallite populations melts one after the other, as the sample temperature reaches their melting temperatures. The amount of energy required to melt these 
crystallites is fixed as is their melting temperature. This means that if one wants to melt them twice as fast (i.e. the heating rate is doubled) the rate of energy input must be twice as fast. It follows from this that the heat flow required to melt the crystallites is a linear function of heating rate. Therefore, the enthalpy of melting will be seen in the reversing signal. In a simple case, this type of melting behaviour closely mimics heat capacity. This is discussed in more detail in [12] and the advanced section.

It should be noted that this simple picture breaks down if cooling occurs during the modulation. As we can see from Fig. 1, it is not necessary to have a negative heating rate at any point in a MTDSC experiment: there can simply be faster and slower rates of heating. Having cooling at any point is an option. If cooling does occur then, to maintain linearity, the crystallites must crystallise instantly to form the same structure as before, something that is generally unlikely both because super-cooling is common and crystallisation to form exactly what was present before is uncommon.

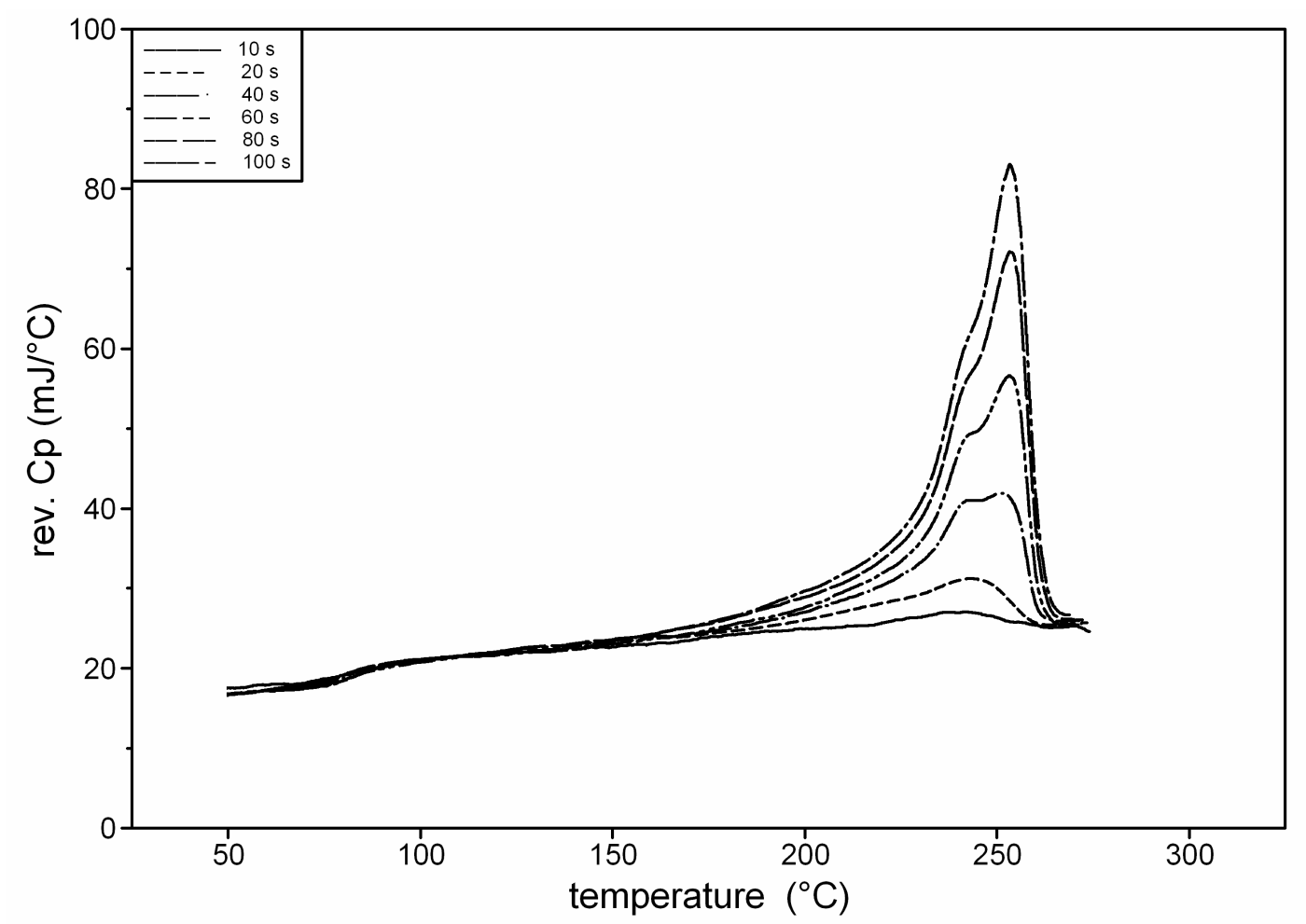

Fig. 21 Effect of period (heating rate $2^{\circ} \mathrm{C} / \mathrm{min}$ ) on reversing heat capacity for semicrystalline PET ('heat-only' conditions, under helium).

Fig. 21 shows some typical results for a semi-crystalline PET. It can be seen that there is a strong frequency dependence of the results in the melt region. The simple model discussed above (from [12]) does not predict that this will occur. Fig. 22 shows how the peak in the melt region is also significantly affected by the underlying heating rate which is again in contradiction 
to the simple model. In both cases the simple model predicts that the reversing signal should be invariant.

In Fig. 3 it can be seen that, above the cold crystallisation temperature, the reversing signal is greater than the average until very near the end of the melting peak. This means that the nonreversing signal is exothermic over most of the melt region. This can be observed in more detail in Fig. 23, which is an enlargement of a selected region of the raw data shown in Fig. 2. Here, at the lowest heating rates (approximately equal to zero), an exotherm is observed within the modulation along with an endotherm at the highest heating rates. At zero heating rate, where the contribution to heat flow from the vibrational heat capacity must be zero, the heat flow is exothermic. This is symptomatic of a rearrangement process. The molten material produced by melting the crystallites with lower melting temperatures can crystallise to form more perfect crystals with a higher melting temperature. This is seen because, at the lower heating rate, the rate of melting is lowest, thus the exothermic process can predominate. At the higher heating rates the reverse is true. In conventional DSC, which provides the same curve as the average signal, there is little or no indication that this rearrangement process is occurring - as the exothermic and endothermic processes cancel each other out. Thus one benefit from using MTDSC is simply the qualitative one that it can make the occurrence of this phenomenon far more apparent.

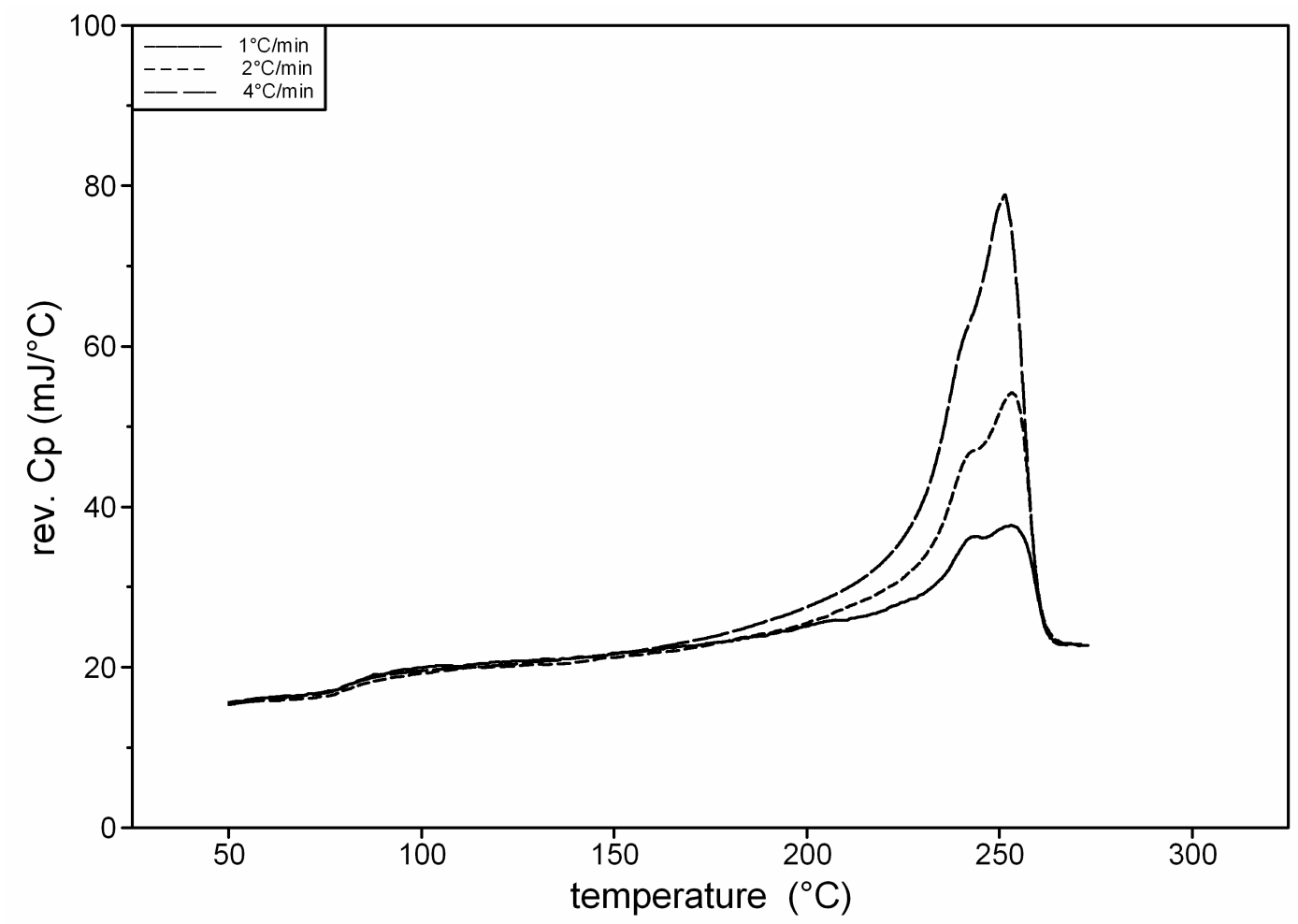

Fig. 22 Effect of heating rate (period 40s, 'heat-only', under helium) on reversing heat capacity for semi-crystalline PET. 
Turning to some simple mathematical representation of melting behaviour, we can express this as follows.

$d Q / d t=\beta\left(C_{p}+g(t, T)\right)$

where $g(t, T)=$ some function that models the contribution to the heat flow from the melting process

When the melting is rapid with respect to the measurement, $g(t, T)$ will be simply a function of temperature, $g(T)$. This means that, in the case of the distribution of crystallites, the melting contribution to heat flow will scale with heating rate exactly like heat capacity if no other process occurs. Taking this simple model gives:

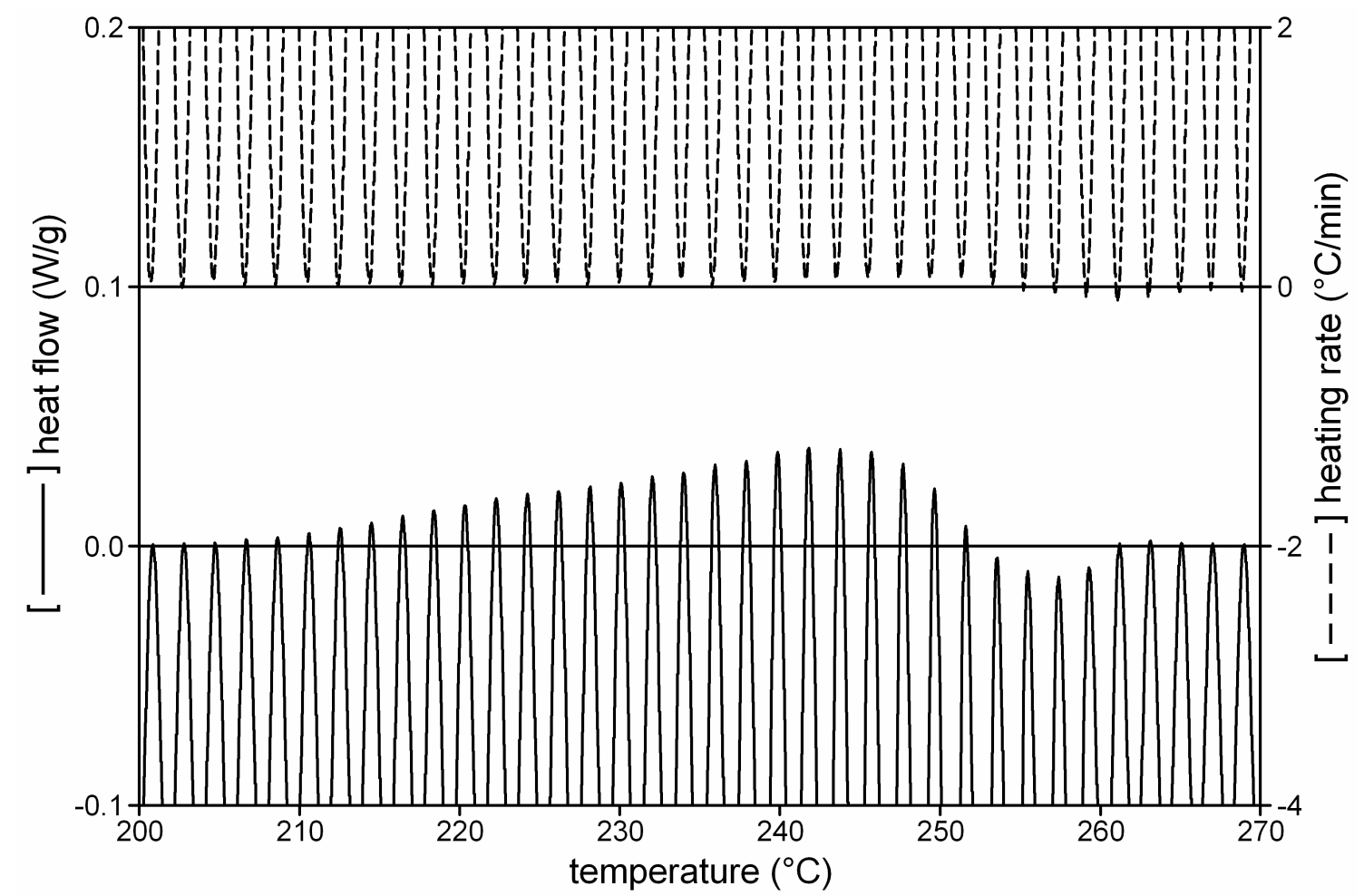

Fig. 23 Enlargement of raw data from Fig. 2 illustrating the exothermic crystal perfection during melting when the heating rate is zero.

$$
\begin{array}{rlrl}
d Q / d t= & \beta\left[C_{p}+\langle g(T)>]\right. & \ldots \text { the underlying signal } \\
& +\left(C_{p}+E\right) B \omega \cos \omega t+C \sin \omega t & & \ldots . \text { the response to the modulation }
\end{array}
$$

where, approximately, $E=\langle g(T)\rangle$ and $C=0$ for this simple case. 
Frequently what is encountered is a complex process that involves melting a population of crystallites with a range of melting temperatures to form molten material which then recrystallise (following some kinetics, thus involving some $f(t, T)$ ) to form a further population of crystallites which then, in their turn, melt and possibly undergo further rearrangement. The data shown in Fig. 23 illustrate this process. To complicate matters further, some workers have suggested that melting is often not rapid with respect to the frequency of the modulation thus there is a time dependency in $g(t, T)[25]$ and $C$ is not zero even without taking account of crystallisation (see the advanced theory section). To allow for this complex range of possibilities it is convenient to define a composite kinetic function that includes all terms other than the heat capacity and models both melting and the kinetics of crystallisation. Viz:

$f(t, T)=g(t, T) d T / d t+f_{2}(t, T)$

Under modulated conditions with no cooling

$f(t, T)=<f(t, T)>+D \sin \omega t+E B \omega \cos \omega$

Equation 58 now becomes

$$
\begin{aligned}
d Q / d t= & \beta C_{p}+\langle f(t, T)\rangle & & \ldots \text { the underlying signal } \\
& +B \omega\left(C_{p}+E\right) \cos \omega t+D \sin \omega t & & \ldots \text { the response to the modulation }
\end{aligned}
$$

Note that the 'reversing' signal during the melt no longer has the same meaning as for an Arrhenius process and the glass transition because it contains a contribution, $E$, from the melting of the crystallites which will typically not be fully reversible due to super-cooling. As noted above, this gives rise to the requirement that there be no cooling at any point during the modulation so that the response does not become asymmetric and thus strongly non-linear.

The question arises as to what might be the form of $f_{2}(t, T)$ and hence $E$ and $D$. At the current state of development, there are no well-established candidates although this may well change in the near future. This point is further discussed in the section dealing with details of the theory.

The situation becomes even more complex when we consider that, even for equation 61 to be true, there must be no significant temperature gradients in the sample. We can reasonably expect that, in the melt region, this will generally not be true. Taking all of these factors into consideration, the melt region is significantly more complex than the other transitions we have considered and it is generally true that a quantitative interpretation of melting behaviour, particularly during experiments with a non-zero underlying heat rate, is not generally possible at present. 


\subsubsection{The measurement of polymer crystallinity}

A problem encountered frequently in determining the crystallinity of a polymer using DSC is that, as illustrated in Figs. 3 and 22, the sample changes its crystallinity during the experiment. The problem becomes one of establishing the initial crystallinity before the experiment started. An understanding of the problem is best approached from the perspective of enthalpy diagrams [31]. Fig. 24 shows an enthalpy-temperature diagram for completely amorphous PET, $100 \%$ crystalline PET, and a 50\% crystalline PET. In the molten state, all of these samples must have the same enthalpy, so the curves obtained for each example are aligned to make this the case. As the diagram indicates, below the melting temperature, the enthalpies are different due to contributions from the latent heat of fusion and the different vibrational heat capacities of the crystal and/or glass compared to that of the liquid.

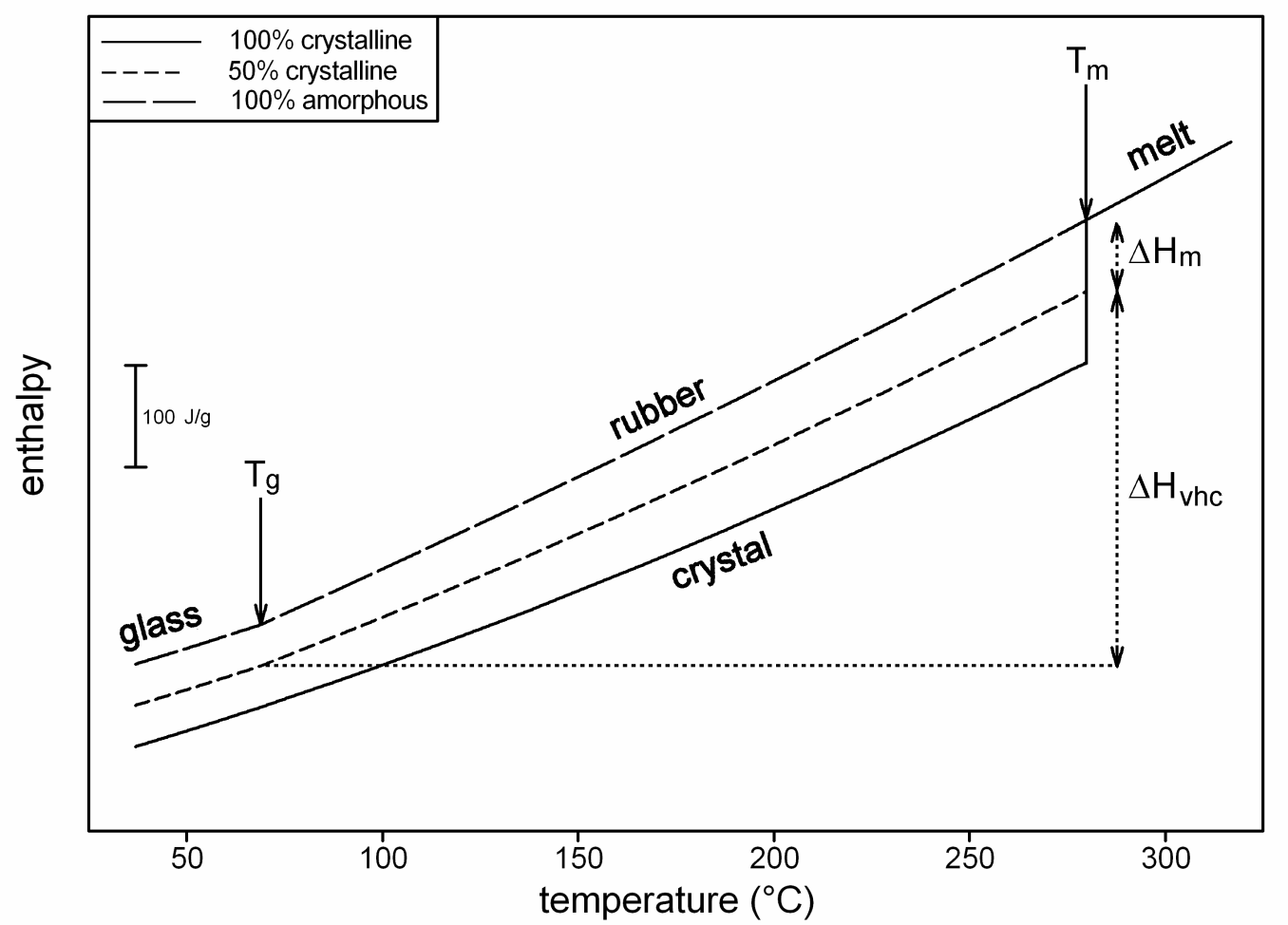

Fig. 24 Enthalpy diagram for $100 \%$ amorphous, $100 \%$ crystalline and $50 \%$ crystalline PET (data from AThAS databank).

The distance between the $100 \%$ amorphous and $100 \%$ crystalline enthalpy line is the enthalpy required to melt a $100 \%$ crystalline sample. This changes with temperature. Consequently there is no unique enthalpy of fusion for a given degree of crystallinity. This must be considered to be function of temperature. A 50\% crystalline material will follow an enthalpy curve approximately half way between the lines defined by 0 and $100 \%$ crystallinity. If one measures the enthalpy change between the equilibrium melting temperature and just above the glass 
transition temperature, this can be broken down into two contributions: one derived from the latent heat, $\Delta H_{m}$ and a contribution from the integral of the vibrational heat capacity, $\Delta H_{v i b}$. The total change in enthalpy can always be measured. If one can estimate the contribution for the vibrational heat capacity of the sample, the difference between this value and the total change in enthalpy will be a measure of the latent heat of melting at the equilibrium melting temperature. The reversing signal can be used to estimate this quantity. It should be noted that this approach solves the problem of the temperature dependence of enthalpy of melting. This is because the total enthalpy (= latent heat of melting plus the enthalpy required to account for the vibrational heat capacity) must be the same when integrating over the whole of the relevant temperature interval regardless of at which temperature the melting (or crystallisation on cooling) occurs.

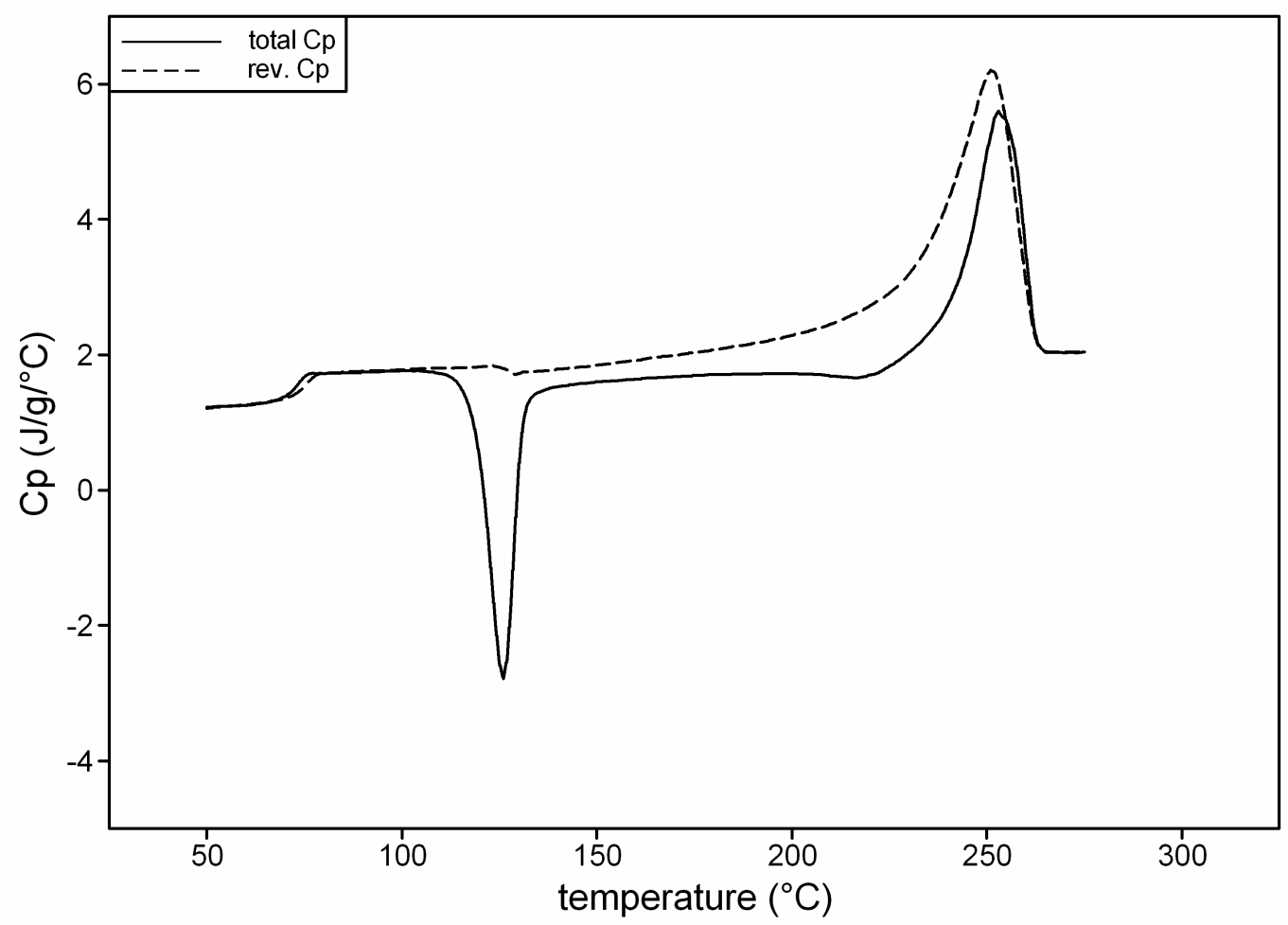

Fig. 25 MTDSC results for quenched PET showing the peak in the reversing signal that comes from the reorganisation process that occurs after the cold crystallisation (underlying heating rate: $2^{\circ} \mathrm{C} / \mathrm{min}$, period $60 \mathrm{~s}$, amplitude: $0.318{ }^{\circ} \mathrm{C}$, under nitrogen).

Starting by considering the simplest case of a purely amorphous polymer, Fig. 25 shows again the results for quenched PET. The simple deconvolution procedure has been used (thus the phase lag has been neglected) and the non-reversing signal has not been calculated. One can consider the reversing signal in isolation as shown in Fig. 26. The broad peak that is seen from 
about $150^{\circ} \mathrm{C}$ is not due to vibrational heat capacity, but arises from the contributions made by the melting and rearrangement process that occur as the sample is heated as discussed above. In Fig. 26, an attempt is made to correct for this by interpolating a baseline to approximate the vibrational heat capacity that would have been measured had crystallisation not occurred. This "corrected" signal can then be re-plotted with the total signal and difference between them (Fig. 27). This difference, when integrated, provides a measure of the enthalpy of melting which is, in this example, zero (to within experimental error). This simple case does not require the use of modulation to estimate the appropriate baseline heat capacity, however, in more complex cases the use of modulation can provide a distinct advantage. Note that, because the peak in the reversing signal is eliminated, the use of the phase lag is irrelevant. Furthermore, had a different frequency or heating rate been used, thus changing the area under the reversing peak during melting, this would also have made no difference to the calculation of crystallinity for the same reason.

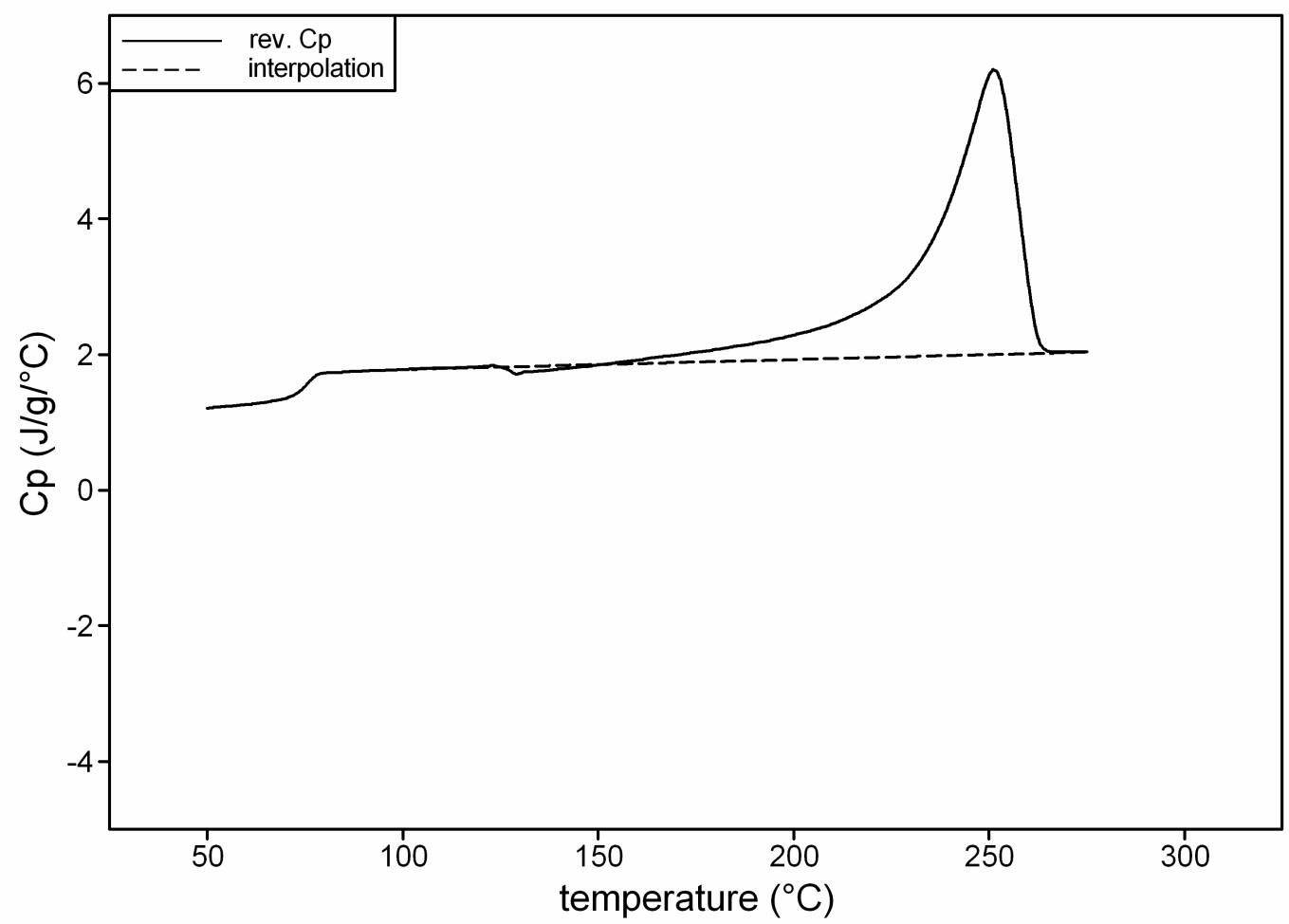

Fig. 26 The reversing $C_{p}$ from Fig. 25 with the interpolation that seeks to approximate the true vibrational heat capacity of the material before crystallisation and rearrangement.

Mathematically we can express these measurements as follows:

$\Delta \mathrm{H}_{\mathrm{u}}=\left(1-X_{c}\left(T_{1}\right)\right) \int \mathrm{C}_{\mathrm{p}, \mathrm{a}} d T+X_{c}\left(T_{1}\right) \cdot \int \mathrm{C}_{\mathrm{p}, \mathrm{x}} d T+X_{c}\left(T_{1}\right) \cdot \Delta H^{o}\left(T_{2}\right)$ 
where $T_{1}=$ some temperature before melting begins

$T_{2}=$ the equilibrium melting temperature of a $100 \%$ crystalline sample

$\Delta H_{u}=$ the enthalpy of melting of the unknown sample

$X_{c}=$ fraction crystallinity before heating

$C_{p, a}=$ the heat capacity of the amorphous material

$C_{p, x}=$ the heat capacity of the crystalline sample

$\Delta H^{\circ}=$ the enthalpy of fusion of a $100 \%$ crystalline sample at $T_{2}$

With the interpolation procedure we are attempting to estimate the quantity $\Delta H_{v i b}=(1-$ $\left.\mathrm{X}_{\mathrm{c}}\left(T_{1}\right)\right) \int C_{p, a} d T+\mathrm{X}_{\mathrm{c}}\left(\mathrm{T}_{1}\right) \cdot \int C_{p, x} d T$. When this is subtracted from the total signal (i.e. to calculate the non-reversing signal), this gives $X_{c}\left(T_{1}\right) \cdot \Delta H^{o}\left(T_{2}\right)_{\mathrm{f}}$ and so, provided $\Delta H\left(T_{2}\right)_{f}$ is known, the crystallinity at the start of the experiment.

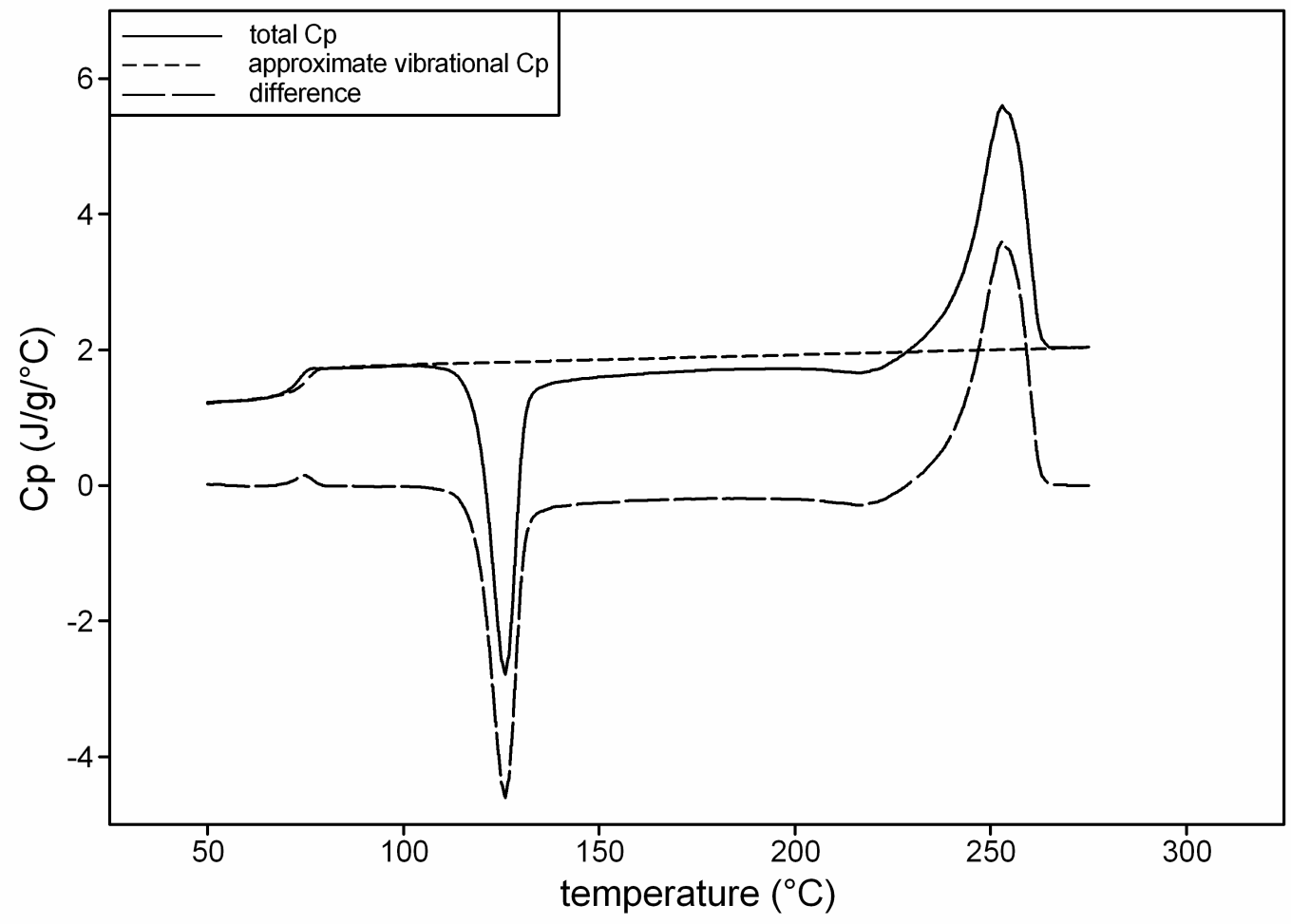

Fig. 27 Co-plot of total heat capacity with the approximated vibrational heat capacity obtained from the reversing $C_{p}$ together with the difference between these two signals. The integral of the difference gives the enthalpy associated with the latent heat of melting.

Fig. 28 shows a blend of polycarbonate (PC) with PET. The glass transition of the amorphous PC occurs beneath the crystallisation peak of the PET and this complicates the interpretation of the data considerably. Again, the contribution from the rearrangement and melting of the 
PET can be removed by interpolation to produce the approximated vibrational heat capacity baseline shown in Fig. 29. The difference signal estimates the crystallinity of the PET as $2.5 \%$, close to the correct value of zero. There is an error that arises from the difference between the reversing and total signal at the glass transition (see above). If this is corrected for, then the apparent crystallinity becomes $1 \%$ i.e. the correct answer to within the typical accuracy for this kind of measurement. In this example, $\Delta H_{v i b}$ is the vibrational heat capacity of everything except the component that can crystallise and/or melt in the temperature range of interest. For an absolute value for crystallinity the mass fraction of this component must be known. Failing this relative crystallinity can be assessed.

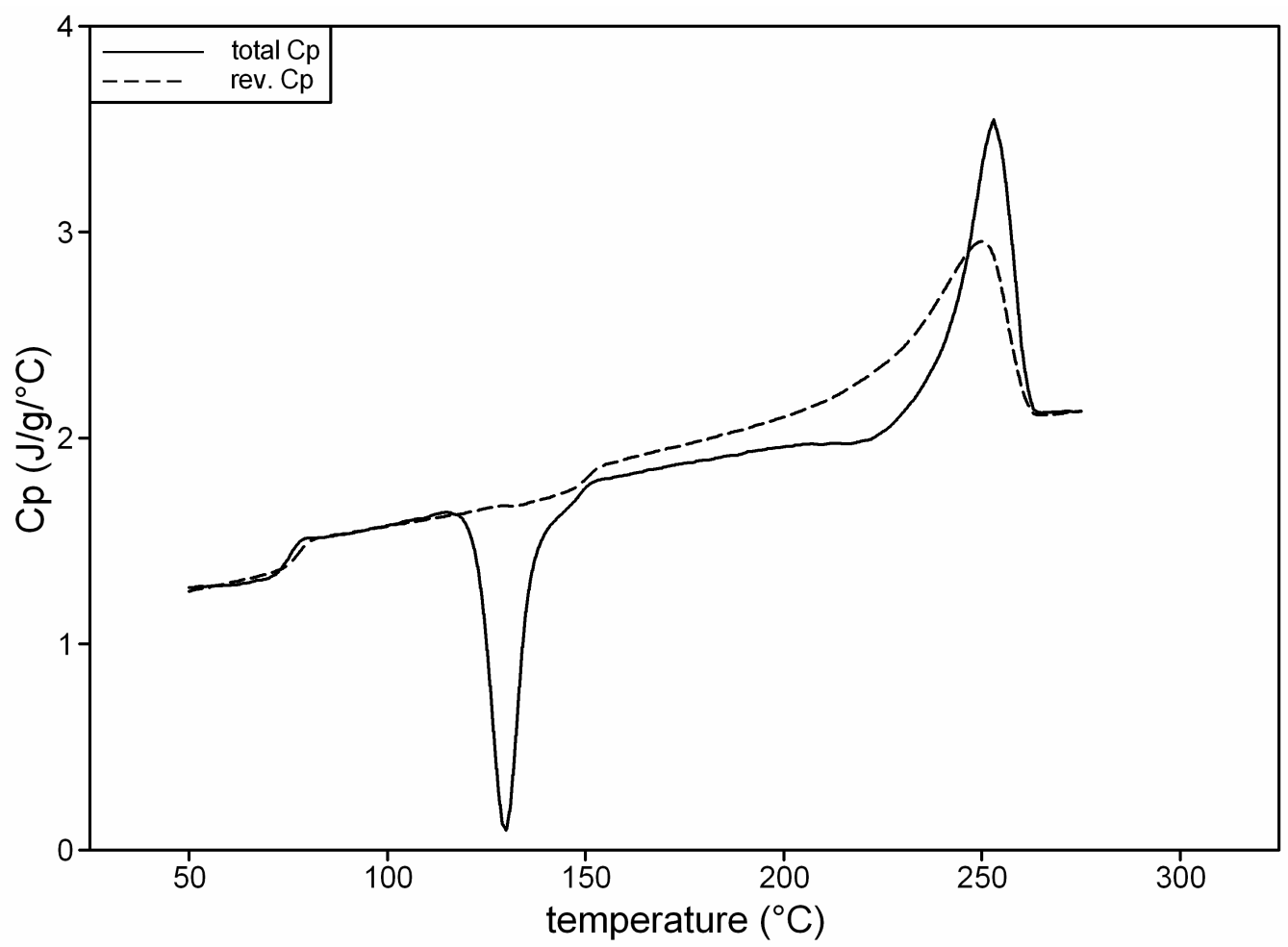

Fig. 28 MTDSC results for a blend of PET and polycarbonate (underlying heating rate: $2^{\circ} \mathrm{C} / \mathrm{min}$, period $60 \mathrm{~s}$, amplitude: $0.318^{\circ} \mathrm{C}$, under nitrogen).

There are assumptions in this approach. Probably the most important is that the interpolation assumes the value for the heat capacity of the sample at the upper temperature is the same as the amorphous polymer. For a 50\% crystalline sample, for example, the vibrational heat capacity at $T_{2}$ would not be the same as a $100 \%$ amorphous sample, which is, in effect, the assumption that is made in this method. When the level of crystallinity is low, then the error from this source will be small. As crystallinity increases the potential error increases, but the fact that, for most polymers, the liquid and crystalline vibrational heat capacities converge around the equilibrium melting temperature tends to reduce this problem. For PET at $48 \%$ 
crystallinity the error from this source has been estimated to be $1.5 \%$. A fuller description of this method can be found in [37].

There are other approaches to measuring polymer crystallinity based on conventional DSC [31]. They use database values for the crystalline and amorphous heat capacity sometimes in combination with extrapolation procedures. Where they can be applied, these methods might be preferred as they make fewer assumptions than the MTDSC approach described above. However, industrially useful systems are often blends and/or contain fillers or other additives, so complex samples are frequently encountered. When this is the case, the more conventional approaches will either produce significant errors or will simply be inapplicable. Under these circumstances MTDSC can offer very real advantages.

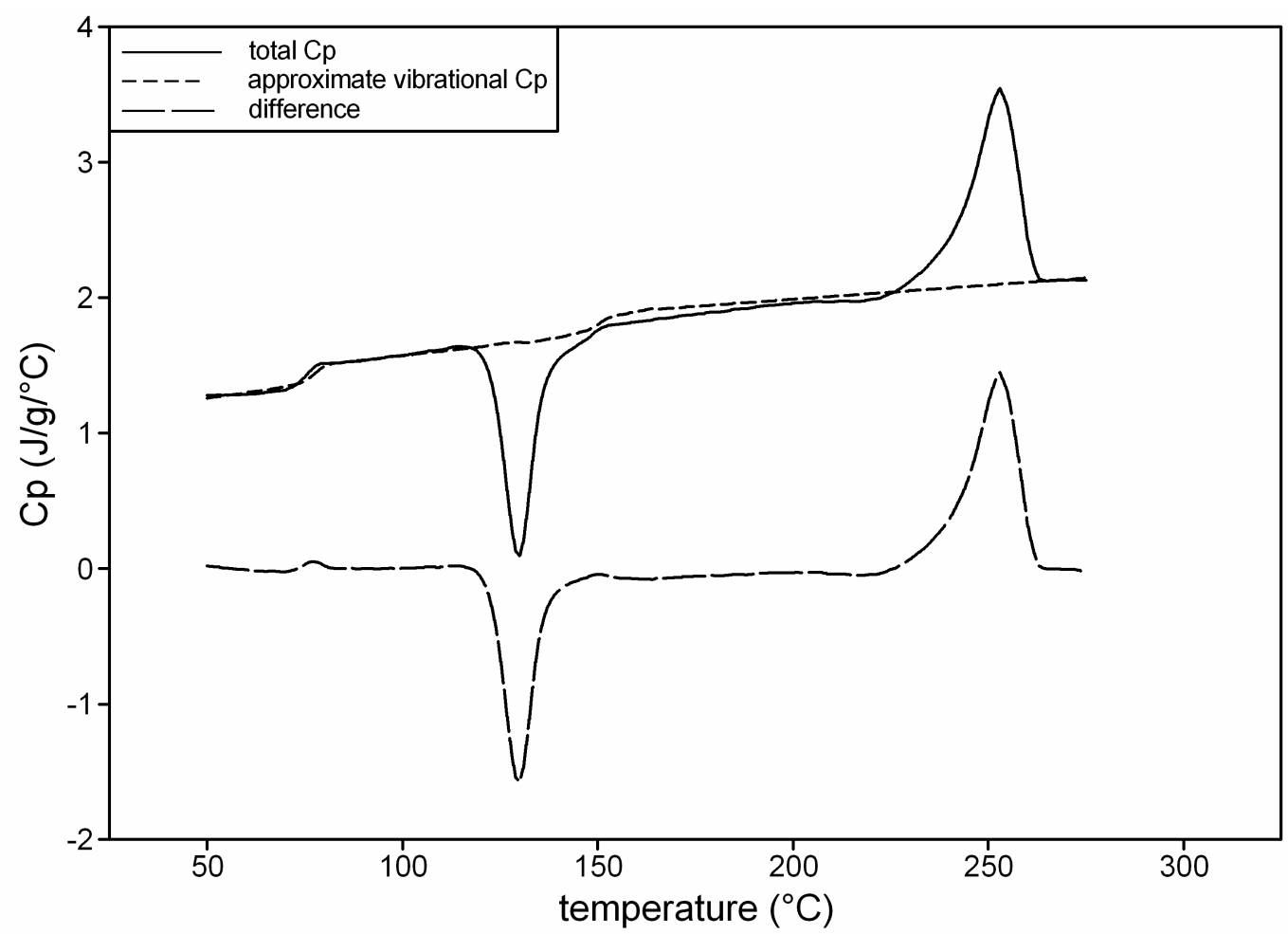

Fig. 29 Data from Fig. 28 with interpolated approximation for the vibrational heat capacity and the difference between them.

\subsubsection{Summary}

- Because polymer melting is the result of a distribution of species all melting at their equilibrium melting temperature, the enthalpy of melting is found in whole or in part in the reversing signal. In principle, where there is no cooling during the modulation cycle, this type of melting behaves in a similar manner to heat capacity. Consequently the reversing signal no longer has the same meaning as it does when considering chemical reactions and glass transitions because the reversing signal contains a contribution 
from an essentially non-reversing process. Clearly, it could be argued that this means the nomenclature for the different signal should be changed. However, this nomenclature is now so well established that it seems better to accept it, while bearing in mind that it is somewhat misleading in the case of melting and rearrangement.

- When, during an experiment that has an underlying heating rate, part of the modulation cycle causes cooling to occur, super-cooling will often mean that the response to the modulation is distorted. Consequently, this type of experiment is generally inadvisable. Conditions should be selected that are 'heat only'.

- Quasi-isothermal experiments in the melt region can often reveal a wealth of information and this is dealt with in detail in chapter 4 .

- When rearrangement is occurring as the sample is heated, the lower heating rates cause an exothermic response balanced by the endothermic response at the higher heating rates. This causes the reversing heat capacity to be higher than the total heat capacity. This behaviour is a good indication that rearrangement is occurring whereas conventional DSC often provides little indication that this is happening. This is because the endothermic and exothermic processes largely cancel each other out.

- The additional information given by MTDSC, compared to conventional DSC, can help in quantifying crystallinity more accurately.

\subsection{CALIBRATION}

\subsubsection{Calibration of the total and reversing signals}

The calibration of the average, or total heat flow, or heat capacity is carried out in the same way as conventional calorimetry because this signal is the same as that normally obtained in conventional un-modulated DSC (see [19]). Briefly, an empty-pan baseline run is carried out where the DSC contains empty pans. This is then subtracted from a run in which sapphire is used as the sample. Sapphire is a good calibrant because it undergoes no transitions in the temperature range covered by most DSC's used to study polymers. The apparent heat capacity of the sample can then be calculated by dividing the empty-pan corrected heat flow by the average heating rate. The heat capacity of sapphire as a function of temperature has been established to high accuracy [38]. Thus, these known values can be compared with the apparent values calculated in the foregoing experiment. Correction factors can then be calculated as a function of temperature. In this way, errors that arise from an imperfect baseline and those derived from inaccuracies in the calibration can be corrected for. In subsequent experiments the sapphire is replaced by a sample in a pan of matched weight. Note that the weight of the pan is matched to that used during the calibration, not necessarily that of the reference pan (see below the discussion on calibrating the reversing signal). The same empty-pan baseline is subtracted and the correction factors are applied to calculate the sample heat capacity as a function of temperature. 
The calibration of the reversing heat capacity is also carried out using a heat capacity standard such as sapphire. The procedure is basically the same as that used for calibrating for heat capacity in a conventional DSC experiment described above. A baseline run is carried out with empty pans then the sapphire calibrant is used. After applying a correction for the emptypan baseline (see below) to the heat capacity measurements, comparisons are made between the apparent values for the heat capacity calculated from equation 13 and the known heat capacity of sapphire. Correction factors can then be derived as a function of temperature. Sometimes a one point (i.e. at one temperature ) calibration is offered in commercial software. This then applies the same correction factor over the whole temperature range. This approach should be used with caution as the correction factors can change significantly with temperature. Note, as shown in Fig. 7, correction factors can be strongly influenced by the period of the modulation. The reasons for this and methods of overcoming them are dealt with in the discussion on advanced theory. Here one is considering the best, simple approach that will work with comparatively long periods. In MTDSC, both of the above calibrations are carried out simultaneously using the same empty-pan baseline and sapphire experiments. Any change to the experimental conditions such as modulation period, heating rate etc. necessitates recalibration.

The empty-pan baseline correction is somewhat more complex for the reversing measurement than for the conventional measurement. This is because, if the cell-plus-pan asymmetry is such that the reference side has an apparent heat capacity that is greater than the sample side, then this represents a heat capacity deficit that must be added to, not subtracted from, the measured value for the sample heat capacity. Because the cyclic heat capacity is derived from a ratio of amplitudes there can never be a negative value. Thus the problem is posed, how does one know when to subtract and when to add the empty pan baseline? In principle, the phase lag will be 180 degrees out of phase when the reference pan has a higher heat capacity than the sample pan. However, many factors can affect the phase lag and, in general, relying on this signal is inadvisable. Probably the simplest approach is to use a lighter-than-average pan in the reference position and measure the sample pan weight in order to ensure that it is heavier. In this way, the empty-pan baseline can be systematically subtracted from any measurement. Note that the sample pan weight must be matched to that used during the calibration.

Sometimes the empty-pan baseline correction for heat capacity is omitted for the reversing signal because, when closely matched sample and reference pans are used, it is usually small. Whether this is adequate depends on the type of information being sought. For example, if all that is required is the glass transition temperature, then a full heat capacity calibration may be excessive. However, as an absolute minimum, a calibration must be performed to obtain a correction factor for the cyclic heat capacity at one temperature in the range of interest.

It has become common practice to present MTDSC results both as heat flow and heat capacity within the same data set. Typically, the greatest source of error is that from the empty-pan baseline for the average signal. Many workers, in order to save time, choose not 
to make this correction and then, almost by default, the total signal is presented as heat flow rather than heat capacity, because this correction has not been made. This is not necessarily bad practice. The experimenters may well be able to obtain the information they require without this additional calibration step. Consequently, one must take care when reading the literature to ascertain what types of data reduction and calibration have been carried out. The questions that must be asked are as follows:

- Has an empty-pan baseline correction been carried out on the reversing and/or total measurement?

- Has the heat capacity calibration constant been determined at a single temperature or as a function of temperature?

- Has the phase lag correction been applied when calculating the reversing signal?

- What type of baseline correction has been applied to the phase lag signal?

If some or all of the answers to the above questions are absent, this implies that a less than optimal procedure has been adopted. One must form one's own judgement as to whether this lack undermines the conclusions that are drawn for a particular case.

It has been discussed in section 2.1.1 and illustrated in Fig. 4, how it can be useful to force the reversing signal to be equal to the total signal in non-transition regions. When doing this, one must decide which signal to take as the accurate one. If no empty-pan correction has been made on the total signal, then the reversing signal would usually give the more reliable value. However, it should be noted that an empty-pan correction is generally desirable to avoid the effect of baseline curvature which can significantly confuse interpretation even when forcing agreement, as illustrated in Fig. 4, is used because this method assumes a linear offset.

Where empty-pan corrections have been made, and long periods (60 seconds or more) are used, this implies low underlying heating rates - as there must be many modulations over each transition. Generally these conditions would still mean the reversing signal is more accurate, as low heating rates give poorer quantification in the total signal. However, a very thick sample would mean the reversing signal could be significantly in error, because of the effects of sample thermal conductivity (see the advanced theory section), while the accuracy of the total signal is improved because the sample is large. Shorter periods make the reversing signal less quantitative and mean higher heating rates can be used - thus making the total signal more reliable. Where accurate values for the heat capacities are needed (rather than differences between the total and the reversing) there is, in reality, no simple answer to suit all cases. Experimenters are encouraged to gain experience with their particular materials. Making accurate heat capacity measurements in non-transition temperature regions using long periods (typically 100 seconds) can do this. Although there is evidence that MTDSC can be used to obtain more accurate heat capacity measurements than conventional methods (see chapter 4), the benefits of MTDSC are mostly achieved through the additional signals it provides through the reversing and non-reversing signals. 


\subsubsection{Comments on methods of phase lag correction}

In much of the practical section on MTDSC it is assumed that the calorimeter behaves ideally. In other words; the sample and sensor can respond instantaneously and there are no significant thermal lags in any part of the system including the sample. In reality, this is clearly not true and there is a discussion of this in the advanced section. The signal that is, in some ways, most affected by non-ideality is the phase signal. In an ideal calorimeter it would be zero except in a transition region. However, this is never the case in practice. The first and simplest solution to this was first proposed by Reading who used a simple interpolation between the start and end of a transition region to provide the baseline that is subtracted from the phase angle to provide the 'true' phase angle [16].

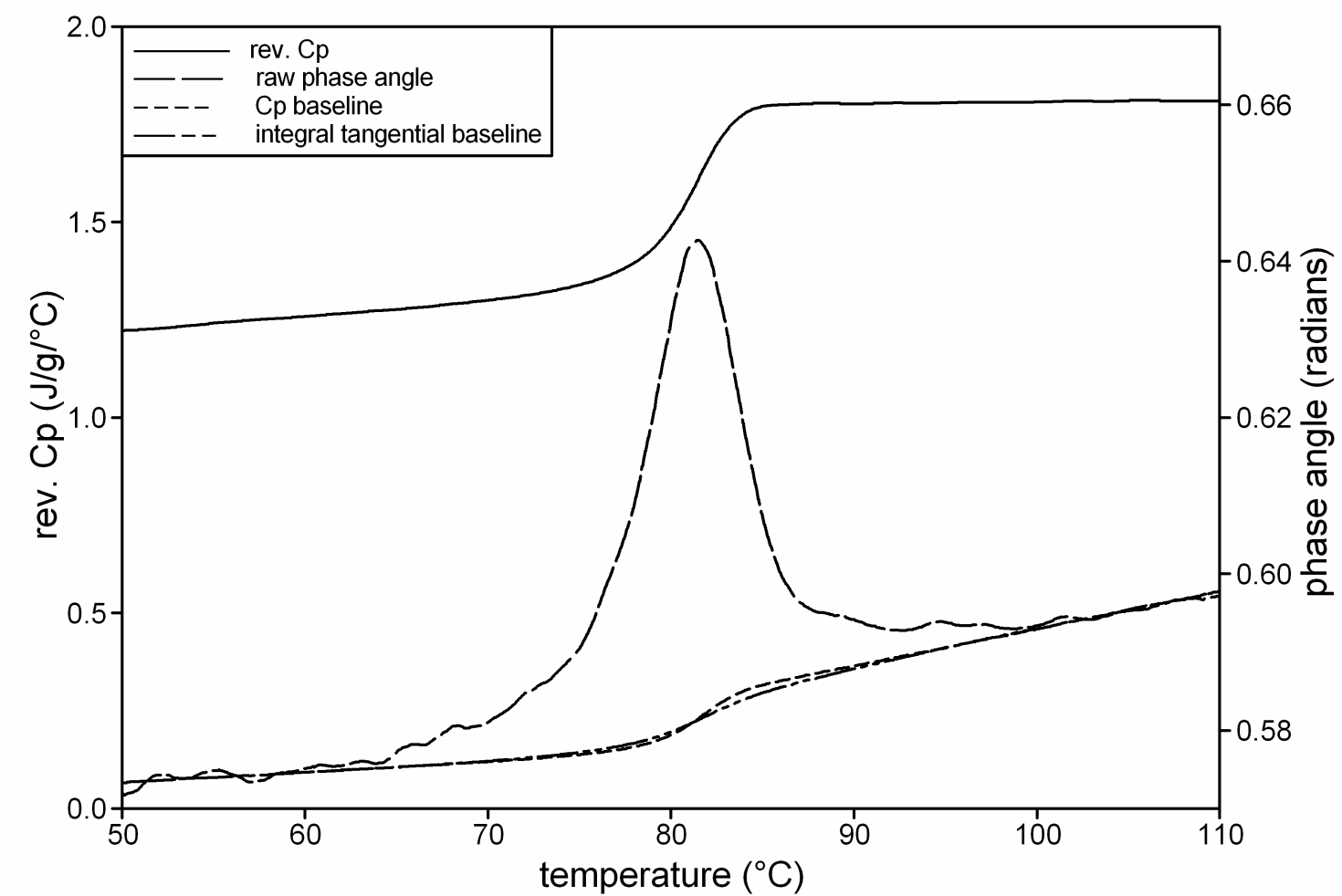

Fig. 30 Constructions showing baseline correction of phase angle during glass transition (PET).

In modelling the non-ideal calorimeter, the simplest non-ideality that we might consider is the thermal resistance that exists between the sensor and the sample pan. It has been shown that when this resistance is significant, the phase angle is affected by heat capacity changes in the sample. Thus, not only is the phase non-zero, it changes with changing sample heat capacity [12]. This poses a problem that can most clearly be seen in the glass-rubber transition. The 
baseline phase lag before and after the transition are not the same. After the transition, the phase can be greater because the heat capacity is greater, see Fig. 30. One solution is to construct a baseline that takes its shape from the reversing heat capacity [39] using the idea that it tracks the phase shift due purely to changes in heat capacity rather than the kinetics of the transition. It is these kinetics which, as discussed below, are what dictates the 'true' value of the phase angle during a transition under ideal conditions. This method of baseline construction is well founded except that in 'real' samples, other things are often changing during the course of the transitions. For example; changing contact resistance between the sample and the pan. Consequently it is often observed that the change in the phase angle between the start and end of the transition goes the opposite way to that dictated by this simple model [16].

By extension of the original method proposed by Reading, the construction of an interpolated sigmoidal baseline, using the well-established integral tangential method normally adopted for constructing baseline under peaks in DSC experiments [40], can also be applied to the phase angle. This has been found to give reliable results even in cases where the phase shift 'goes the wrong way'. The two different approaches to deriving a baseline are shown in Fig. 30 for the case of a 'well behaved' glass transition. In this case the shift in phase lag baseline follows the expected trend. The two baselines are almost identical. Consequently, either approach can be used. The interpolation method has the advantage that it is more robust to non-ideal behaviour. Both methods are, in fact, equivalent if it is considered that the fractional area under the phase angle peak is a measure of the extent to which the transition is complete. In some cases the phase angle correction is applied over a large temperature range, as, for example in Fig. 2. In such circumstances a simple linear interpolation is advisable.

The simplest approach, and one that in practice is often justified, is to not carry out the phase lag correction and make the approximation as has already been mentioned above, i.e. $C^{*}=$ $C_{p R}$. In many practical cases, knowing $C_{p K}$ or $C\left(=C_{p K} \beta\right)$ is not of any value. Thus, it can simply be neglected.

\subsection{OVERVIEW}

The results of the deconvolution process shown in Fig. 4 can now be discussed in terms of the simple theory offered above. Outside of transition regions, the total signal and the reversing signal should be the same and the kinetic heat capacity should be zero. At the cold crystallisation the reversing signal is not greatly affected, thus the non-reversing peak contains the enthalpy of crystallisation and one observes a negative peak in the kinetic heat capacity. This is in accordance with the simple theory outlined above. At the glass transition, the reversing signal shows the step change in heat capacity expected at $T_{g}$. The non-reversing signal shows a peak. This is, at first glance, in accordance with the simple theory because the enthalpy loss on annealing is recovered during the transition, but not quickly lost again, thus this aspect of the glass transition is non-reversing. However, this interpretation must be made 
within the context of the frequency dependence of the glass transition as would be expected from dynamic mechanical and dielectric measurements. Over the melt region there is a complex behaviour that cannot be accounted for by a simple theory for melting. However, a qualitative description allows one to identify crystalline rearrangement when it occurs in the reversing signal, even when it is not easily discerned in the total signal (equivalent to conventional DSC). One can also exploit the additional information provided by the modulation to enable a more accurate measure of initial crystallinity to be made in complex samples. In all cases, whether studying kinetic processes like cure or crystallisation, investigating blends by looking at their glass transitions or measuring polymer crystallinity, the modulation offers significant advantages. The remaining chapters of this book illustrate this point very well.

\section{Detailed Discussion of the Theory of MTDSC}

\subsection{INTRODUCTION}

Under the heading of this more detailed discussion of the theory of MTDSC, we will again consider the following types of transitions and phenomena.

- Chemical reactions and related processes.

- Frequency-dependent heat capacity and glass transitions.

- First-order phase transitions.

In each case, the full derivations of the expressions used in the more practical exposition above will be given. The discussion will also look more closely at some of the simplifying assumptions and the problems that arise when these no longer apply. Before this some comments are made on alternative modulations and deconvolution methods.

\subsection{MODULATION AND DECONVOLUTION}

It is possible to use multiple sine waves [10] and so extract as a Fourier series (or other deconvolution procedure) the response to several frequencies simultaneously, as illustrated in chapter 4. An extension of this is the use of saw-tooth temperature modulations [20]. These can be considered to be a combination of an infinite series of sine waves (though only a limited range will be available in practice). A symmetric saw-tooth (same heating and cooling rate) only has odd harmonics, but an asymmetric saw-tooth (different heating and cooling rates) is equivalent to a broad range of frequencies.

The use of averaging combined with a Fourier transform is by no means the only possible deconvolution procedure [17]. Details of a linear fitting approach have been published that could easily be adapted to deal with some forms of non-linear behaviour [10]. Other multi- 
parameter fitting approaches are possible. However, overwhelmingly, the current practice is to use a single sinusoidal modulation and a Fourier transform so this will be the focus of this chapter and this book.

\subsection{CHEMICAL REACTIONS AND RELATED PROCESSES}

For a zero-order chemical reaction (including a term for the contribution to the heat flow from heat capacity), the rate of the reaction is dependent only on temperature. Thus, it produces heat at a rate given by some function of temperature $h(T)$. Taking the heating programme given in equation 6:

$d Q / d t=C_{p}(\beta+\omega B \cos \omega t)-h\left(T_{0}+\beta t+b \sin \omega t\right)$

For an exactly linear case, $h(T)=h_{1}+h_{2} T$

$$
\frac{d Q}{d t}=\left(C_{p} \beta-h_{1}-h_{2} T_{0}-h_{2} \beta t\right)+B\left(C_{p} \omega \cos \omega t-h_{2} \sin \omega t\right) .
$$

More generally, $h\left(T_{0}+\beta t+B \sin \omega t\right)$ can be expanded as a power series:

$$
h\left(T_{0}+\beta t\right)+B h^{\prime}\left(T_{0}+\beta t\right) \sin \omega t+\frac{B^{2}}{2} h^{\prime \prime}\left(T_{0}+\beta t\right) \sin ^{2} \omega t+\ldots
$$

so

$$
\frac{d Q}{d t}=\left(C_{p} \beta-h\left(T_{0}+\beta t\right)\right)+B\left(C_{p} \omega \cos \omega t-h^{\prime}\left(T_{0}+\beta t\right) \sin \omega t\right)-\frac{1}{2} B^{2} h^{\prime \prime}\left(T_{0}+\beta t\right) \sin ^{2} \omega t+\ldots
$$

Should $B$ be small enough for terms in $B^{2}, B^{3}$, to be negligible in comparison with $B$, the response of the heat flow is effectively linear, and $d Q / d t$ agrees with that for the exactly linear case. In the event that the amplitude of the temperature modulation $B$ is not so small, the terms in $B^{2} \sin ^{2} \omega t, B^{3} \sin ^{3} \omega t$ are significant and the higher harmonics $\cos 2 \omega t, \sin 3 \omega t, \ldots$ appear in the modulation and the heat flow $d Q / d t$. These can, in principle, give information about the kinetic law $h$ through its derivatives $h^{\prime \prime}, h^{\prime \prime \prime}, \ldots$

Returning to the linear case:

$<d Q / d t>=C_{p} \beta-h_{1}-h_{2} T_{0}-h_{2} \beta t$

modulation of the heat flow $=B\left(C_{p} \omega \cos \omega t-h_{2} \sin \omega t\right)=A_{H F} \cos (\omega t-\phi)$ 
where $A_{H F}=B C_{p} \omega \sqrt{1+h_{2}^{2} / C_{p}^{2} \omega^{2}}, \phi=-\tan ^{-1}\left(h_{2} / C_{p} \omega\right)$

$$
\mathrm{A}_{\mathrm{H} F}=\omega B
$$

In the absence of the kinetic process $(h=0)$ a measurement of the heat capacity is:

$$
C_{p}=A_{H F} / A_{H R}
$$

This indicates that:

$$
\begin{aligned}
& A_{H F} / A_{H R}=C^{*} \\
& C^{*} \cos \phi=C_{p R} \\
& C^{*} \sin \phi=C_{p K}
\end{aligned}
$$

could all be useful measured quantities as indicated above. For the general zero-order linear reaction, $C^{*}=C_{p} \sqrt{1+h_{2}^{2} / C_{p}^{2} \omega^{2}}$ differs from the true heat capacity, although not significantly if the frequency is high enough. The phase-corrected reversing heat capacity, however, is given by the following relation:

$$
C_{p P C R}=C^{*} \cos \phi=C_{p}
$$

At the same time, the non-reversing heat flow is simply

$$
<\frac{d Q}{d t}>-\beta C^{*} \cos \phi=\text { Average heat flow }-\beta C_{p P C R}=h(T)
$$

Thus, it is possible to separate the contribution in the total heat flow from the heat capacity and that which arises from the zero order reaction.

Considering a more general process that gives rise to a heat flow, and is governed by a kinetic function that is dependent on temperature and time, viz, for some process;

$d Q / d t=f(t, T)$

By analogy with the simpler case considered above:

$d Q / d t=\beta C_{p}+B C_{p} \omega \cos \omega t+f(t, T)$ 


$$
\simeq\left(C_{p} \beta-f\left(t, T_{0}+\beta t\right)\right)+B\left(C_{p} \omega \cos \omega t-\frac{\partial f}{\partial T}\left(t, T_{0}+\beta t\right) \sin \omega t\right)
$$

neglecting the non-linear (higher-order) terms. Following the above procedure leads to an average heat flow:

$$
\begin{aligned}
<d Q / d t> & =C_{p} \beta+f\left(t, T_{0}+\beta t\right)+O\left(B^{2}\right) \\
& =C_{p} \beta+f(t, T)+O\left(B^{2}\right)
\end{aligned}
$$

an amplitude of the heat-flow modulation:

$$
A_{H F}=B C_{p} ? \sqrt{1+\left(\frac{\partial f}{\partial T} / C_{p} ?\right)^{2}}+O\left(B^{2}\right)
$$

and a phase lag:

$\phi=\tan ^{-1}\left(\frac{\partial f}{\partial T} / C_{p} \omega\right)+O(B)$

Neglecting the higher-order terms:

$$
C_{p P C R}=C_{p}, C_{P K}=\frac{\partial f}{\partial T} / \omega \text { and } C^{*}=C_{p} \sqrt{1+\left(\frac{\partial f}{\partial T} / C_{p} \omega\right)^{2}}
$$

where $\frac{\partial f}{\partial T}$ means $\frac{\partial f}{\partial T}\left(t, T_{0}+\beta t\right)$ so it is the average value.

Also, using the complex notation, the heat-flow modulation is just

$$
B \omega \operatorname{Re}\left\{\left(C_{p}-i \frac{\partial f}{\partial t} / \omega\right) e^{i \omega t}\right\}=B \omega \operatorname{Re}\left\{\hat{C} e^{i \omega t}\right\}
$$

where $\hat{C}=C_{p}-i \frac{\partial f}{\partial T} / \omega$.

Thus,

$C_{p P C R}=C_{p}$ and $C_{p K}=(\partial f / \partial T) / \omega$.

Considering the Arrhenius equation viz; 
$\frac{d Q}{d t}=\beta C_{p}+H f(x) A e^{-E / R T}+C_{p} B \omega \cos \omega t$,

where

$H A e^{-E / R T}=H A e^{-E /\left(R T_{0}+\beta t\right)}+\left.\left(\frac{d}{d T}\left(H A e^{-E / R T}\right)\right)\right|_{T=T_{0}+\beta t} B \sin \omega t+$ higher-order terms

and the reaction extent satisfies equation 79:

$\frac{d x}{d t} / f(x)=A e^{-E / R\left(T_{0}+\beta t+B \sin \omega t\right)}$

This last equation, because the temperature variation is taken to be sufficiently small, leads to:

$x=\langle x\rangle+$ oscillatory term

where $\langle x\rangle$ is the same as the reaction extent during conventional DSC and the oscillatory term takes the form:

$\frac{x_{c}(t) B}{\omega} \cos \omega t+$ higher-order terms and terms in $\frac{B}{\omega}$

the coefficient $x_{\mathrm{c}}$ being independent of the modulation.

The combination of all of these eventually results in a total heat-flow signal:

$\frac{d Q}{d t}=C_{p} \beta+<H f(x) A e^{-E / R T}>\quad \ldots \ldots \ldots . .$. the underlying signal
+
$B\left(\left(\omega C_{p} \cos \omega t+f(<x>)\left(\frac{d}{d T}\left(H A e^{-E I R T}\right)\right)_{T=T_{0}+\beta t} \sin \omega t+\frac{d f}{d x}(<x>) H A e^{-E / R\left(T_{0}+\beta t\right)} \frac{x_{c}}{\omega} \cos \omega t\right)\right.$

+ higher-order terms

the response to the modulation

$=C_{p} \beta+H f(<x>) A e^{-E / R<T>}+$ higher-order terms $\quad$........the underlying signal 
$+B\left(\omega C_{p} \cos \omega t+\omega C_{P K} \sin \omega t+\frac{D}{\omega} \cos \omega t\right) \ldots .$. the response to the modulation

+ higher harmonics and other higher-order terms.

The higher-order terms are those in $B^{2}, B^{3}$, etc. which appear because of the non-linearity of the kinetic process.

Under usual operation, the temperature variation should be small enough and the frequency high enough for terms involving $B^{2}$ or $1 / \omega$ to be negligible. The various signals can then be related to the heat capacity and kinetics according to:

Average heat capacity $=C_{p}+H f\left(\langle x>) e^{-E / R<T>} / \beta\right.$

Phase corrected reversing heat capacity $=C_{p P C R}=C_{p}$

Non-reversing heat capacity $\quad=\left\langle d Q / d t>/ \beta-C_{p R}=H f\left(\langle x>) e^{-E / R<T>} / \beta\right.\right.$

Kinetic heat capacity $=C_{P K}=\frac{f(<x>)}{\omega} \frac{d\left(H A e^{-E I R T}\right)_{T=<T>}}{d T}$

Of course, with $\omega$ large, the last is small compared with the reversing heat capacity and the response of the sample is dominated by the heat capacity of the sample.

The above analysis of MTDSC data in terms if kinetics has been proposed and developed by Lacey and Reading and co-workers $[2,5,9,12,14]$ and also by Toda and co-workers $[42,43]$. It is essentially a kinetic approach to MTDSC theory and this is the basis of the theory throughout this chapter.

\subsection{FREQUENCY DEPENDENT HEAT CAPACITY AND THE GLASS TRANSITION}

Heat content stored in molecular motions, such as vibration, is assumed to be rapid when compared with the modulation of the temperature; kinetic effects influence heat flow but only through reaction rates. This contrasts with cases where the heat in molecular vibrations itself is not rapid and some sort of kinetics plays a role in the heat capacity, or at least in some of the heat taken up and released: a relaxation time appears in the rate of change of enthalpy.

Perhaps the simplest example of how a time scale can be involved in heat flow to and from a sample, and thereby give an (apparent) dependence of heat capacity upon frequency, is where the thermal conductivity of the sample is in some sense poor (or, equivalently, the sample's specific heat is very large). For simplicity, we may look at the case of a homogeneous spherical sample inside a locally uniform part of the calorimeter. More realistic cases are less easy to analyse but the qualitative effects are much the same. 
Using complex notation, the cyclic part of the temperature can be written as:

$\tilde{T}=\operatorname{Re}\left\{\hat{T}(r) e^{i \omega t}\right\}$

where $r$ distance from the centre of the sample and $\hat{T}(R)=\hat{T}_{s}$ on the sample's surface $r=R$; by $T_{s}$ we now must be quite precise and here we mean the temperature on this surface. From consideration of heat flow inside the sample:

$\frac{d^{2} \hat{T}}{d r^{2}}+\frac{2}{r} \frac{d \hat{T}}{d r}=\frac{i \omega \rho c}{\kappa} \hat{T}$

where $\rho=$ density

$c=$ specific heat

$K=$ thermal conductivity

(all assumed constant here). This complex temperature $\hat{T}$ can then be found to be:

$\hat{T}=\hat{T}_{s} R \sinh \left(\sqrt{\frac{\omega \rho c}{2 \kappa}}(1+i) r\right) r \sinh \left(\sqrt{\frac{\omega \rho c}{2 \kappa}}(1+i) R\right)$

and the total power flow into the sample is:

$$
\begin{aligned}
\frac{d Q}{d t} & =\operatorname{Re}\left\{4 \pi R^{2} \times \kappa \frac{\partial \tilde{T}}{d r}\right\} \quad(\text { area } \times \text { heat flow/unit area }) \\
& =\operatorname{Re}\left\{4 \pi R \kappa\left(\sqrt{\frac{\omega \rho c}{2 \kappa}}(1+i) R \operatorname{coth}\left(\sqrt{\frac{\omega \rho c}{2 \kappa}}(1+i) R\right)-1\right) \hat{T}_{s} e^{i \omega t}\right\}
\end{aligned}
$$

This gives rise to a complex heat capacity of:

$$
\hat{C}=-4 i \pi \frac{R \kappa}{\omega}\left(\sqrt{\frac{\omega \rho c}{2 \kappa}}(1+i) R \operatorname{coth}\left(\sqrt{\frac{\omega \rho c}{2 \kappa}}(1+i) R\right)-1\right)
$$

For sufficiently small samples, $R \sqrt{\frac{\omega \rho c}{\kappa}}<<1$, this expression simplifies to the true specific heat: 
$\hat{C} \simeq C_{p}=\frac{4 \pi R^{3}}{3} \rho c$

If the frequency is insufficiently small, $\hat{C}$ will deviate significantly from $C_{p}$ as indicated. Estimates on how large the frequency may be taken for specific sizes of samples, still with desired accuracy, can be found in papers by Hatta [24] and Toda [25]. These effects can be used to measure the specimen's thermal conductivity.

In the rest of the discussions on transitions throughout this chapter, such size-dependent effects are taken to be negligible. Interpretation of results is easiest if the sample is small enough for its temperature to be uniform, $T_{s}(t)$.

A more interesting case of where heat capacities, cyclic, phase-corrected reversing, etc., vary with frequency $\omega$ is of a material undergoing a phase transition. This time, the size of sample is not so important - the phenomenon is an intrinsic property of the material - and the underlying temperature $\langle T\rangle=T_{0}+\beta t$ plays a key role.

With a relaxation time that decreases rapidly as temperature increases, heat is gained and lost more easily at higher temperatures. In particular, for temperature $T>T_{g}$, the glass-transition temperature, $d Q / d t=C_{p l} d T / d t$, where $C_{p l}$ is the liquid heat capacity. For lower temperatures, relaxation is slow and the heat capacity is $C_{p g}$, the heat capacity of the glass, which is smaller than $C_{p l}$. This observed heat capacity depends on how the relaxation time compares with the timescale of the changing temperature: roughly speaking, time scale > relaxation time $\left(T_{g}<T\right)$ leads to $C_{p l}$, while time scale $<$ relaxation time $\left(T_{g}>T\right)$, gives $C_{p g}$. As a consequence, even for standard DSC, $T_{g}$ depends on the temperature ramp $\beta$; more specifically the glass-transition temperature increases with $\beta$.

This carries over to the cyclic measurements. Provided that the relaxation time is small enough compared with the period of oscillation, $2 \pi / \omega C^{*} \simeq C_{p l}$, while if the period of oscillation is too short, $C^{*} \simeq C_{p g}$. The change occurs at a glass transition temperature dependent on the frequency of modulation, $\omega$. By the same reasoning as for standard DSC, and consequently the total signal in MTDSC, this cyclic-glass transition temperature increases with $\omega$. Moreover, because the oscillations must, in some sense, be fast, as noted above, in MTDSC the cyclic $T_{g}$ is higher than the underlying $T_{g}[32]$.

How this $T_{g}$ varies with ramp $\beta$ and frequency $\omega$ depends upon the nature of the relaxation process. A specific model, with the enthalpy of a specimen changing according to a single ordinary differential equation and affected by both temperature and its rate of change, is given in equation 42. Such models lead to predictions of not just the glass transition temperatures but also the profiles of $C^{*}, C_{P P C R}$ and $C_{P K}$ in the transition regimes. MTDSC offers a way of 
determining key physical parameters related to the material, not just $C_{p l}$ and $C_{p g}$, from comparing the variation of $T_{g}$ 's with $\beta$ and $\omega$, and transition profiles, with those predicted by such models.

A more general approach to materials exhibiting frequency-dependent-heat capacities is that of Schawe and co-workers [18]. For some linear (or at least, for temperature not varying too much, approximated linear) process, the rate of heat intake and rate of change of temperature can be related through a convolution:

$\frac{d Q}{d t}=\int_{-\infty}^{t} \psi\left(t-t^{\prime}\right) \frac{d T}{d t}\left(t^{\prime}\right) d t^{\prime},=\int_{-\infty}^{\infty} \psi\left(t-t^{\prime}\right) \frac{d T}{d t}\left(t^{\prime}\right) d t^{\prime}$

taking $\psi\left(t^{\prime}\right) \equiv 0$ for $t^{\prime}<0$, for some kernel $\psi$ which is fixed by the underlying physical process. For a simple material with $Q=C_{p} T$ and $C_{p}=$ constant, $\psi\left(t^{\prime}\right)=C_{p} \delta\left(t^{\prime}\right)$ where $\delta\left(\mathrm{t}^{\prime}\right)$ is the "Dirac delta function": $\delta\left(t^{\prime}\right)=0$, for $t^{\prime} \neq 0, \int_{-a}^{a} \delta\left(t^{\prime}\right) d t^{\prime}=1$ for $\mathrm{a}>0$. $)$

Taking the Fourier transform of this convolution leads to:

$$
F\left(\frac{d Q}{d t}\right)\left(\omega^{\prime}\right)=F(\psi)\left(\omega^{\prime}\right) F\left(\frac{d T}{d t}\right)\left(\omega^{\prime}\right)
$$

where $F(f)\left(\omega^{\prime}\right)=\int_{-\infty}^{\infty} f(t) e^{i \omega^{\prime} t} d t$.

Writing $\omega=-\omega^{\prime}$ leads to:

$$
F\left(\frac{d Q}{d t}\right)(-\omega) / F\left(\frac{d T}{d t}\right)(-\omega)=\hat{C}(\omega)=F(\psi)(-\omega)
$$

the Fourier transform of the rates of change of enthalpy and temperature are related through that of the kernel function which can be identified with the complex heat capacity. By carrying out a succession of experiments to determine $\hat{C}(\omega)$ and then doing a Fourier inversion it is then possible, in principle, to recover the function $\psi$ and hence gain information about the physical kinetics. However, to be useful this approach must refer to more specific models of realistic behaviour which then brings us back to the kinds of results discussed in this chapter. The basic model used for the glass transition is that of Hutchinson and Kovacs [41] (see also [31])

$$
\frac{d \delta}{d t}=-\Delta C_{p} \frac{d T}{d t}-\frac{\delta}{\tau_{0}} e^{-\Delta h^{*} / R T}
$$


Here $\delta=$ enthalpy - equilibrium enthalpy $=$ enthalpy $-C_{p l} T$. This equation can be rewritten in terms of the difference between the enthalpy and that for the glass,

$\eta=$ enthalpy $-C_{p g} T=\delta+T \Delta C_{p}$

Near the average glass transition the Arrhenius term can be approximated as

$$
e^{-\Delta h^{*} / R T}=e^{-\Delta h^{*} / R T_{g \beta}} \cdot e^{\Delta h^{*}\left(T-T_{g \beta}\right) / R T_{g \beta}^{2}}
$$

and the equation for $\eta$ becomes, on making use of this "large activation energy" approximation $\left(\Delta h^{*} \gg R T_{g \beta}\right.$ so terms in $R T_{g \beta} / \Delta h^{*}$ can be neglected)

$$
\frac{d \eta}{d t}=\left(\frac{1}{\tau_{0}} \exp \left(\frac{-\Delta h^{*}}{R T_{g \beta}}\right)\right) \exp \left(\frac{\Delta h^{*}\left(T-T_{g \beta}\right)}{R T_{g \beta}^{2}}\right)\left(T_{g \beta} \Delta C_{p}-\eta\right)
$$

This equation indicates that $\eta$ is the size of $T_{g \beta} \Delta C_{p}$, while $T$ changes an amount of size $R T_{g \beta}{ }^{2} / \Delta h^{*}$ and does so, for the averaged measurements, at rate $\beta$. Balancing the terms in this equation leads to an expression for the glass transition temperature:

$\frac{\beta \Delta h^{*}}{R T_{g \beta}^{2}}=\frac{1}{\tau_{0}} \exp \left(\frac{-\Delta h^{*}}{R T_{g \beta}}\right)$

The solution of the approximate equation for $\eta$ leads, eventually, to an ageing term as well as an integral term which. In Section 3.5 an ad-hoc model is used for the average signal in order to more directly illustrate points with regard to the phenomenology of this measurement.

Looking at the cyclic parts of $\eta, \quad \operatorname{Re}\left\{\hat{\eta} e^{i \omega t},\right\}$ and of temperature, $\quad \operatorname{Re}\left\{\hat{T} e^{i \omega t}\right\}$ is seen that:

$$
i \omega \hat{\eta}=\left(\frac{1}{\tau_{0}} \exp \left(-\frac{\Delta h^{*}}{R T_{g \omega}}\right)\right) \exp \left(\frac{\Delta h^{*}\left(T-T_{g \omega}\right)}{R T_{g \omega}^{2}}\right)\left(\frac{\Delta h^{*} \Delta C_{p} T \hat{T}}{R T_{g \omega}^{2}}+\hat{T} \Delta C_{p}-\frac{\bar{\eta} \Delta h^{*} \hat{T}}{R T_{g \omega}^{2}}-\hat{\eta}\right)
$$

where the averaged temperature, written at $T$ is within the order of magnitude $R T_{\mathrm{g \omega}}{ }^{2} / \Delta h^{*}$ of the cyclic transition temperature $T_{g \omega}$. For high frequencies, so that the cyclic transition 
temperature is significantly greater that the averaged one, the averaged enthalpy difference , is exponentially close to $T$. Thus

$$
\left(1+i \omega \tau_{0} \exp \left(\frac{\Delta h^{*}}{R T_{g \omega}}\right) \exp \left(\frac{-\Delta h *\left(T-T_{g \omega}\right)}{R T_{g \omega}^{2}}\right) \hat{\eta}=\hat{T} \Delta C_{p}\right.
$$

The location of the glass transition is then fixed by:

$\omega \tau_{0} \exp \left(\frac{\Delta h^{*}}{R T_{g \omega}}\right)=1$

while the response to the modulation is:

$$
\frac{\hat{\eta}}{\hat{T}}=\frac{\Delta C_{p}}{1+i \exp \left(\frac{-\Delta h^{*}\left(T-T_{g \omega}\right)}{R T_{g \omega}^{2}}\right)}
$$

Taking the real and imaginary part predicts, according to this model,

$$
\Delta C_{p P C R}=\frac{\Delta C_{p}}{1+\exp \left(\frac{-2 \Delta h^{*}\left(T-T_{g \omega}\right)}{R T_{g \omega}^{2}}\right)} \text { and } C_{p K}=\frac{\Delta C_{p} \exp \left(\frac{-\Delta h^{*}\left(T-T_{g \omega}\right)}{R T_{g \omega}^{2}}\right)}{1+\exp \left(\frac{-2 \Delta h^{*}\left(T-T_{g \omega}\right)}{R T_{g \omega}^{2}}\right)}
$$

respectively.

A slightly more general formulation of this Hutchinson-Kovacs model [41] is given in Chapter 4. In that model, for $C_{p g}$ and $C_{p l}$ assumed constant, the equilibrium enthalpy is given by $C_{p l} T=C_{p g} T+\varepsilon_{h} N^{*}$ with $N^{*}(T)$ the equilibrium number of configurations of energy $\varepsilon_{h}$. More generally, the enthalpy $=Q=C_{p l} T+\delta=C_{p g} T+\varepsilon_{h} N$, with $N(t)$ the instantaneous number of configurations. Using the relation between $N^{*}$ and $\mathrm{T}$, the relaxation law $d N / d t=\left(N^{*}-N\right) / \tau$ (see Chapter 4) gives, on eliminating $N$ in favour of $\delta$, the above equation for excess enthalpy $\delta$. 


\subsection{MELTING}

One simple model for melting, which has shown good agreement with experimental results, is to represent it in the same way as an irreversible, endothermic chemical reaction (see above). For a polymer consisting (partly) of crystals with a range of melting temperatures, $T_{m}$, at any time $t$ the crystalline mass distribution can be given in terms of a density function $m\left(t, T_{m}\right)$ (so the total mass fraction of crystals is $x(t)=\int m\left(t, T_{m}\right) d T_{m}$ ). During melting (and ignoring possible recrystallisation), the crystalline density reduces according to a rate law of the form:

$\frac{\partial m}{\partial t}=F\left(T, T_{m}\right) m$

C.f. equations 38 and 39 [23]. As in the above consideration of Arrhenius kinetics, the cyclic signal then takes the approximate form:

$\omega B\left(C_{p} \cos \omega t+C_{P K} \sin \omega t\right)$

Thus the reversing heat capacity gives a good estimate of $C_{p}$. The kinetic heat capacity, which decreases as the reciprocal of modulation frequency, is determined by how the melting rate depends upon temperature:

$$
C_{P K}=\frac{L}{\omega} \int\left(m\left(t, T_{m}\right) \frac{\partial F\left(T, T_{m}\right)}{\partial T}\right) d T_{m}
$$

where $L$ is the latest heat for the transition $\times$ mass of sample.

A more accurate consideration of the reversing heat capacity (still following the Arrhenius analysis, or again see Toda et al. [23,25]) will give its variation with frequency (the difference $C_{P P C R}-C$ decreases as $1 / \omega^{2}$ for "large" frequency $\omega$ ).

A very different approach to polymer melting stems from the detailed description by Wunderlich [31]. In this, for the time scales involved in the calorimetry, melting is considered as instantaneous: the fraction $m\left(t, T_{m}\right)$ drops to zero as $T(t)$ increases through $T_{m}$. (Melting is not kinetically hindered; or if it is, its time scale is very short compared with that of the MTDSC.) Nucleation of crystals (which take the form of lamellae) is still taken to be negligible (the time scale for this is long compared with an experiment) but now existing crystals (with $T_{m}>T$ ) grow when either there is available melt or melt is being made available through the melting of smaller lamellae (with lower values of $T_{m}$ ). In this model, a simple 
version of which has been formulated and discussed in Lacey \& Nikolopoulos [45], the number densities $n\left(t, T_{m}\right)$ of lamellae stays fixed until $T$ exceeds $T_{m}$ while initially the mass fraction $m\left(t, T_{m}\right)$ increases in a well-determined way, again until $T$ exceeds $T_{m}$, when both $n$ and $m$ falls to zero.

In the particular model considered by Lacey and Nikolopoulos [45], there were three distinct phases:

1) the small initial rate of melting of crystals allows instant recrystallisation of the melted polymer, so there is no net melting: both the average and phase-corrected reversing heat capacities remain equal to $C_{p}$;

2) there is now sufficient crystalline material being melted for net melting to occur for part of each cycle but insufficient for recrystallisation not to take up the excess melt before the cycle is complete; thus there is no melting on average so the average signal only manifests the heat capacity while the cyclic heat capacity is now increased due to the melting than crystallisation in each cycle ;

3) an increased crystalline fraction (and reduced number of surviving lamellae for melt to recrystallise onto) means that net melting occurs throughout each cycle and both average and phase-corrected heat capacities exceed $C_{p}$.

A more quantitative analysis of the model for this third phase shows that, if the amplitude of modulation is sufficiently small for the temperature to be always increasing:

$$
\begin{array}{ll}
\text { Average heat capacity } & =C_{p}+\text { latent heat } \times \text { mass } \times \\
& \left(m\left(t, T_{0}+\beta t\right)-\text { fractional recrystallization rate } / \beta\right) \\
& =\text { cyclic heat capacity } \\
\text { phase-corrected heat } & C_{p}+\text { latent heat } \times \text { mass } \times m\left(T_{0} \beta t, T_{0}+\beta t\right) \\
\text { capacity } &
\end{array}
$$

(to leading order) so the average heat capacity is less than the phase-corrected heat capacity. (With a larger amplitude, with temperature decreasing for a part of each cycle, the expression for the phase-corrected heat capacity is rather more complicated due to the melting rate not being sinusoidal - it is zero whenever the temperature falls. In this case the phase-corrected heat capacity can drop below the average heat capacity towards the end of the phase.) The above relation for small amplitude agrees with the observation that the phase-corrected reversing heat capacity is higher than the average heat capacity. The model, as currently constituted, does not allow for the fact that real polymers almost never achieve $100 \%$ crystallinity thus there is always a substantial amorphous fraction that, in effect, cannot crystallise. This could be accounted for by describing a 'background' amorphous fraction that participates to a lesser extent than the fraction closer to the lamellae and thus more able to crystallise. The apparent frequency dependence of the melting and rearrangement peak in, for example, PET shown in Fig. 21 has its origins, in our view, in thermal transport difficulties within the sample. This could be accounted for by allowing for either the sample temperature 
dropping below the programme $T_{0}+\beta t+B \sin \omega t$ when melting occurs or for the sample to have to have an internal temperature that varies significantly during the phase change. Each of these effects will act to hinder the melting and produce the crossing of the underlying and phase-corrected heat capacities. Toda's concept of kinetic hindering of melting [23.25] can also be interpreted in the same way (i.e. as arising from temperature differences rather than true kinetics of melting) and so this approach might be combined with that of [45] particularly near the end of melting where the phase-corrected reversing heat capacity is below that of the average signal.

\subsection{CALIBRATION}

About the simplest model for an ideal calorimeter, i.e. one which is unbiased and has perfect measurements of both sample and reference (but which nevertheless allows for direct heat transfer between sample and reference), is due to Wunderlich [46]. Heat flow to the sample is given by the temperature difference between the reference and the sample and that between block and sample:

$$
\left(C_{R}+C_{p}\right) \frac{d T_{s}}{d t}=K_{1}\left(T_{R}-T_{s}\right)+K_{2}\left(T_{F}-T_{s}\right)
$$

where $C_{R}$ is the heat capacity of the actual pan and its environs. Similarly,

$$
C_{R} \frac{d T_{R}}{d t}=K_{1}\left(T_{s}-T_{R}\right)+K_{2}\left(T_{F}-T_{R}\right)
$$

The similarity between these two equations is associated with the lack of bias. This sort of ordinary-differential-equation model relies on the calorimeter being adequately represented by a finite number of parts (here two) each of which has a uniform temperature. The heat-transfer coefficients will be independent of temperature for a truly linear system (but the device can be regarded satisfactorily as linear as long as their values do not change significantly over the temperature range inside the calorimeter at any instant or from the minimum to the maximum of a modulation). Eliminating $T_{F}$, the model reduces to

$$
C_{R} \frac{d \Delta T}{d t}+K \Delta T=C_{p} \frac{d T_{s}}{d t}
$$

where $\Delta T=T_{R}-T_{s}$ and $K=2 K_{1}+K_{2}$.

For $T_{s}=T_{0}+\beta t+B \sin \omega t=T_{0}+\beta t+\operatorname{Re}\left\{-i B e^{i \omega t}\right\}$, 


$$
\begin{aligned}
\Delta T & =\beta C_{p} / K+\operatorname{Re}\left\{\omega B C_{p} e^{i \omega t} /\left(K+i \omega C_{R}\right)\right\} \\
& =\beta C_{p} / K+\frac{\omega B C_{p}}{K^{2}+\omega^{2} C_{R}^{2}}\left(K \cos \omega t+\omega C_{R} \sin \omega t\right) \\
& =\frac{\beta C_{p}}{K}+\frac{\omega B C_{p}}{\sqrt{\left(K^{2}+\omega^{2} C_{R}^{2}\right)}} \cos (\omega t-\varphi)
\end{aligned}
$$

where "phase lag" $\varphi=\tan ^{-1}\left(C_{R} / K\right)$.

A single calibrating run with a specimen of known heat capacity in a sample pan then suffices to find:

the value of $K$ used in the average signal, underlying heat capacity $=K<\Delta T>/ \beta$;

the value of $C_{R}$ used, for instance, in obtaining the cyclic heat capacity = $\sqrt{\left(K^{2}+\omega^{2} C_{R}^{2}\right)} \times$ amplitude of $\Delta T / \omega B$.

It is apparent that there are three pieces of data available for finding just two device unknowns, $K$ and $C_{R}$. The calibrating factor $\sqrt{\left(K^{2}+\omega^{2} C_{R}^{2}\right)}$ and phase lag $\varphi$ are fixed by $K, C_{R}$ and the frequency $\omega$ so even if the calorimeter is to be used with different modulation periods further calibration is unnecessary - according to this model.

More sophisticated models, with a greater number of calorimeter parts, their temperatures and interconnections lead to similar relationships between $\Delta T$ and $C_{p}$ but involve more internal device parameters. It follows that if a more complicated model of the calorimeter is required, a greater number of calibrating runs is needed to fix the calorimeter constants before the calibrating factor for the cyclic heat capacity, and the phase lag, will be known for any frequency.

Allowances for bias or for imperfect temperature measurement can be made with simple variations of the Wunderlich model [46].

Considering first imperfect measurements, the temperatures registered by the thermocouples are:

$$
\begin{aligned}
& T_{s M}=(1-\eta) T_{s}+\eta T_{F}=T_{o}+\beta t+B \sin \omega t \\
& T_{R M}=(1-\eta) T_{R}+\eta T_{F},
\end{aligned}
$$

where $T_{s}$ and $T_{R}$ are the true sample and reference temperatures. 
The parameter $\eta, 0<\eta<1$, gives a measure of the imperfection of the device. Manipulations similar to those by the basic model lead, taking a very simple case of :

$K_{1}=0, K=K_{2}$,

to:

$<\Delta T>=\beta(1-\eta) C_{p} / K$

and:

$\Delta \tilde{T}=$ cyclic part of $\Delta T=\omega K(1-\eta) C_{p} \operatorname{Re}\left\{\frac{B e^{i \omega t}}{\left(K+i \omega C_{R}\right)\left(K+i \omega \eta\left(C_{R}+C_{p}\right)\right)}\right\}$

where now $\Delta T=T_{R M}-T_{S M}$.

Writing $\Delta \tilde{T}=\operatorname{Re}\left\{\Delta \hat{T} e^{i \omega t}\right\}$,

$\Delta \hat{T}=\frac{K(1-\eta) \omega B C_{p}}{\left(K+i \omega C_{R}\right)\left(K+i \omega \eta\left(C_{R}+C_{p}\right)\right)}$

This indicates a non-linear relation between the modulation of the temperature difference and the heat capacity. However, a single calibration can again be sufficient: the average measurement fixes $k \equiv K /(1-\eta)$. Use of the in-phase and out-of-phase parts of the modulated measurements determine the real and imaginary parts of

$\frac{\omega B C_{p}}{(K /(1-\eta)) \Delta \hat{T}} \equiv a_{1}+i a_{2}=\left(1+i b_{1}\right)\left(1+i b_{2}\right)$

where $b_{1}=\omega C_{R} / K$ and $b_{2}=\omega \eta\left(C_{R}+C_{p}\right) / K$

Then this means that it is possible to find $b_{1}$ (hence $C_{R} / K$ ) by solving a quadratic equation and $b_{2}$. This leads to a second quadratic equation this time, for $\eta$.

All this determines $\eta, K$ and $C_{R}$ from the single calibration provided it is clear, e.g. from past experience, which roots of the quadratic equations are appropriate. If this were not the case, a second run would be necessary. 
Although the relationship between $C_{p}$ and $\Delta \hat{T}$ is more awkward than the simpler, ideal case, the evaluation of the (complex) cyclic heat capacity is not a particular problem (once $\eta, K$ and $C_{R}$ are known):

$$
C_{p}=\hat{C}=\frac{\left(K+i \omega C_{R}\right)\left(K+i \omega \eta C_{R}\right)(\Delta \hat{T} / B)}{\omega\left[K(1-\eta)-\left(K+i \omega C_{R}\right)(i \omega \eta \Delta \hat{T} / B)\right]}
$$

Bias can result either from an asymmetric distribution of heat capacity or from asymmetric thermal conductivities. The simple case of no direct thermal connection between sample and reference, symmetric heat capacity, but uneven heat transfer can be modelled by:

$$
\left(C_{R}+C_{p}\right) \frac{d T_{s}}{d t}=\lambda K\left(T_{F}-T_{s}\right), C_{R} \frac{d T_{r}}{d t}=K\left(T_{K}-T_{R}\right)
$$

ehere $\lambda \neq 1$. (Good temperature measurement is assumed in this model.)

In the present case

$$
\begin{aligned}
& <\Delta \hat{T}>=\frac{\beta}{K \lambda}\left(C_{p}+(1-\lambda) C_{R}\right) \\
& \Delta \hat{T}=\frac{B \omega}{\lambda} \frac{\left(C_{p}+(1-\lambda) C_{R}\right)}{K+i \omega C_{R}}
\end{aligned}
$$

The bias is apparent from the offset term $(1-\lambda) C_{R}$, so that $\Delta T \neq 0$ even when $C_{p}=0$. Now two calibrating runs are needed. For instance, with an empty sample pan, a first run fixes $(1-\lambda) C_{R} / K \lambda$ from the average signal. In a second run, with $C_{p}>0$, the average signal gives $K \lambda$ and the cyclic signal determines $C_{R} / K$. In a subsequent experiment the (complex) cyclic heat capacity measurement is then:

$$
C_{p}=\frac{\lambda\left(K+i \omega C_{R}\right) \Delta \hat{T}}{B \omega}-(1-\lambda) C_{R}
$$

Although these two departures from ideality are themselves somewhat specialised, much more general models lead to rather similar results as will be seen below.

The models based on ordinary differential equations, such as those above, vary in simplicity and accuracy. Some allow for bias and/or temperature measurements differing from true 
temperatures. All are linear. This is the key fact which can be exploited to get general results relating temperature measurements to heat capacities, without making detailed assumptions on heat transfer within the calorimeter. Once linearity holds, calibration, of some type, can be done. (The only drawback in such a general consideration is that how calibration factors depend on frequency is no longer clear: calibration should be done at the particular frequency $\omega$ of the experiment, or at least at similar frequencies so interpolation can be employed.)

The calorimeter (and its contents) can be considered as a body which contains heat (specific heat of its parts and heat capacity of the pans and sample) and which is capable of transferring heat, through forced convection and conduction, in some linear way: flow of heat is proportional to temperature or temperature gradient. Significant non-linear heat transport, for instance due to natural convection or through thermal properties of the calorimeter varying noticeably over the range of temperatures found within the device during a few oscillations, would have a major affect on the ease of use of the method and interpretation of experimental results.

Assuming that the calorimeter does behave linearly, the temperature $T(\mathbf{x}, t)$ satisfies some linear heat equation. The underlying part $\bar{T}$ (or the actual temperature if conventional DSC is being done) then takes the form $\bar{T}=S(\mathbf{x})+\beta t$ for a linear ramp $\beta$, provided that the heating of the calorimeter is controlled through its external underlying temperature being some $\bar{T}_{f}=S_{f}+\beta t$. The same goes for the underlying forcing temperature being position dependent, of the form $\bar{T}_{f}=S_{f}(\mathbf{x})+\beta t$, on part of the calorimeter surface, with the remaining part being perfectly insulated. If the temperature on part of the surface were ambient, say $\bar{T}_{f}$ $=\bar{T}_{0}=$ constant for some points $\mathbf{x}$, the temperature ramp in the calorimeter would be position dependent, $\bar{T}=S(\mathbf{x})+b(\mathbf{x}) t$, and the following discussion of calibration would need to be modified; should this be the case there would tend to be a steady linear drift between the temperatures at two points in the calorimeter, for example the sample and reference temperatures.

Because of the linear equation satisfied by temperature inside the calorimeter and outside the sample and its pan, the temperature at any point, in the case of the steady rise, can be given as a linear combination of $\bar{T}_{f}$ and the sample's temperature $\bar{T}_{s}$. In particular, the underlying temperatures as measured for the sample and the reference pan are given by:

$\bar{T}_{m}=J_{m s} \bar{T}_{s}+J_{m f} \bar{T}_{f}$

$\bar{T}_{r}=J_{r s} \bar{T}_{s}+J_{r f} \bar{T}_{f}$

respectively. All the coefficients $J$ are independent of the ramp $\beta$ (but might dependent weakly on temperature so that the characteristics of a calorimeter might be rather different at 
the finish of a run from what they are at start). The $J$ 's also satisfy $J_{m s}+J_{m f}=J_{r s}+J_{r f}=1$ (if $\bar{T}(\mathbf{x})=\mathrm{S}(\mathbf{x})+\beta \mathrm{t}$ is a possible temperature distribution then so is $\mathrm{S}(\mathrm{x})+\mathrm{Bt}+\mathrm{S}_{0}$ for any constant $\mathrm{S}_{0}$ ). Solving these, $\bar{T}_{s}$ and $\bar{T}_{f}$ can instead be determined in terms of $\bar{T}_{m}$ and $\bar{T}_{r}$ :

$\bar{T}_{s}=J_{s m} \bar{T}_{m}+J_{s r} \bar{T}_{r}$

$\bar{T}_{f}=J_{f m} \bar{T}_{m}+J_{f r} \bar{T}_{r}$

where, again, $J_{s m}+J_{s r}=J_{f m}+J_{f r}=1$ and, what is more, $\bar{T}_{f}=\left(J_{s m}-J_{f m}\right) ? \bar{T}+\bar{T}_{s}$, where ? $\bar{T}=\bar{T}_{r}-\bar{T}_{m}$ is the underlying measured temperature difference.

The underlying rate of heating of the sample, and its pan (and possibly of its environs), $\beta\left(C_{R}+\right.$ $C_{p}$ ), is of course proportional to the temperature difference $\bar{T}_{f}-\bar{T}_{s}$ so:

$\beta\left(C_{R}+C_{p}\right)=K\left(\bar{T}_{f}-\bar{T}_{s}\right)$

and $\beta C_{p}=<\frac{d Q}{d t}>=J_{1} \Delta \bar{T}+J_{2} \beta$

for some constants (or weakly temperature-dependent functions) $J_{1}$ and $J_{2}$.

(Equivalently, solving a (linear) heat equation throughout the interior of the calorimeter with the sample absent, $C_{p}=0$, gives ? $\bar{T} \propto \beta$. Now including the sample gives the same heat equation but with a heat sink $\beta C_{p}$ and so an extra contribution to the temperature difference, one proportional to $\beta C_{p}$, must be included. This again gives:

$\beta C_{p}=<\frac{d Q}{d t}>=J_{1} \Delta \bar{T}+J_{2} \beta$.)

The calorimeter can now be calibrated by doing an empty run to find $J_{2} / J_{1}$, and then a run with a sample of known heat capacity, for example some sapphire, which will determine $J_{1}$ and hence $J_{2}$.

The cyclic signal can be looked at in a very similar way. Taking the cyclic parts of the temperature, for a purely sinusoidal modulation (or any harmonic for a less simple wave form) to be of the form $\widetilde{T}(\mathbf{x}, t)=\widetilde{T}_{1}(\mathbf{x}) \cos \omega t+\widetilde{T}_{2}(\mathbf{x}) \sin \omega t=\operatorname{Re}\left\{\hat{T}(x) e^{i \omega t}\right\}$ for: 
$\hat{T}=T_{1}-i T_{2}$

The measured temperatures and those of the sample and in the exterior are related via:

$\hat{T}_{m}=a_{m s} \hat{T}_{s}+a_{m f} \hat{T}_{f}$

$\hat{T}_{r}=a_{r s} \hat{T}_{s}+a_{r f} \hat{T}_{f}$

or equivalently:

$\hat{T}_{s}=a_{s m} \hat{T}_{m}+a_{s r} \hat{T}_{r}$

$\hat{T}_{f}=a_{f m} \hat{T}_{m}+a_{f r} \hat{T}_{r}$

(For the modulation, it is not too important that no part of the calorimeter's surface be fixed at ambient.) As with the $J$ 's, the $a$ 's should be at most weakly dependent upon temperature, but they will depend upon frequency and no simple relation between pairs should be expected. (For very high frequency, $a_{r s}$ and $a_{r f}$, for example, will both be small.)

The rate of intake of heat by the sample and its surroundings, $\frac{d \tilde{Q}}{d t}=\operatorname{Re}\left\{\hat{Q}_{T} e^{i \omega t}\right\}=\operatorname{Re}\left\{\frac{d}{d t}\left(C_{R}+C_{p}\right) \hat{T}_{s} e^{i \omega t}\right\}=\operatorname{Re}\left\{i \omega\left(C_{R}+C_{p}\right) \hat{T}_{s} e^{i \omega t}\right\}$, is again going to be proportional to the complex cyclic temperature difference $\hat{T}_{f}-\hat{T}_{s}$, now with a factor of proportionality which varies with angular frequency:

$\hat{Q}_{T}=\hat{Q}+i \omega C_{r} \hat{T}_{s}=i \omega\left(C_{p}+C_{r}\right) \hat{T}_{s}=k(\omega)\left(\hat{T}_{f}-\hat{T}_{s}\right)$

Here $\hat{Q}=i \omega C_{p} \hat{T}_{s}$ gives the rate of heat intake by the sample and $i \omega C_{r} \hat{T}_{s}$ by its environs.

Because the $a$ 's do not satisfy the same identities as the $J$ 's and because the different temperatures have rather different rates of change (amplitude and phase vary with position) the expression relating to $\hat{Q}$ to $\hat{T}_{m}$ and $\hat{T}_{r}$ is not quite as simple as the formula for $<\frac{d Q}{d t}>$ :

$$
\begin{aligned}
\hat{Q} & =i \omega C_{p} \hat{T}_{s}=i \omega C_{p}\left(a_{s m} \hat{T}_{m}+a_{s r} \hat{T}_{r}\right) \\
& =k\left(\left(a_{f m}-a_{s m}\right) \hat{T}_{m}+\left(a_{f r}-a_{s r}\right) \hat{T}_{r}\right)-i \omega C_{r}\left(a_{s m} \hat{T}_{f}+a_{s r} \hat{T}_{s}\right)
\end{aligned}
$$

This leads, after a little manipulation, to: 


$$
\Delta \hat{T}=\frac{K_{1}+K_{2} C_{p}}{1+K_{3} C_{p}} \hat{T}_{m}
$$

where the $K$ 's vary with angular frequency $\omega$ (and possibly depend weakly upon temperature).

To do the calibration, for a required frequency, in finding the three $K^{\prime}$, it is now necessary to carry out three runs, for instance one with an empty sample pan to fix $K_{1}$, and then two more with different heat capacities for the sample to determine $K_{2}$ and $K_{3}$. Once these have been established, the calorimeter can be used to determine the $C_{p}$ (or rather the complex heat capacity $\hat{C}$ ) for a sample by :

$$
\hat{C}=\frac{\hat{Q}}{\hat{T}_{s}}=\frac{K_{1} \hat{T}_{m}-\Delta \hat{T}}{K_{3} \Delta \hat{T}-K_{2} \hat{T}_{m}}=\frac{K_{1}-\left(\Delta \hat{T} / \hat{T}_{m}\right)}{K_{3}\left(\Delta \hat{T} / \hat{T}_{m}\right)-K_{2}}
$$

(Note that $K_{1}, K_{2}, K_{3}$ are complex quantities and therefore this relation contains information about the phase lag $\phi$ as well as the cyclic heat capacity $C^{*}$.)

For a standard modulation $\widetilde{T}_{m}=B \sin \omega t$. So $\hat{T}_{m}=-i B$ and

$$
\hat{C}=\frac{i(\Delta \hat{T} / B)-K_{1}}{K_{2}-i K_{3}(\Delta \hat{T} / B)}
$$

With a biased calorimeter, one which indicates non-zero $\Delta T$ even without a sample, both $K_{1}$ and $J_{2}$ are non-zero. Without bias, only the calibrations with known samples need to be done. Rather more of a problem with devices deviating from the ideal is the inaccuracy of the measurement of the sample's temperature: $T_{m} \neq T_{s}$. For the ideal case, $T_{m}=T_{s}$, in the above $J_{m s}=J_{s m}=a_{m s}=a_{s m}=1$ and $J_{m f}=J_{s r}=a_{m f}=a_{s r}=0$. The form, of the underlying measurement remains unchanged:

$$
C_{a}=\text { average heat capacity }=J_{1}(\Delta \bar{T} / \beta)+J_{2}
$$

but the cyclic measurement simplifies to:

$$
\hat{C}=\text { complex heat capacity }=B_{1}(\Delta \hat{T} / B)+B_{2}
$$


that is, $K_{3}=0, \quad B_{1}=i / K_{2}$ and $B_{2}=-K_{1} / K_{2}$. Regarding calibration, in such a case, one of the runs for the cyclic calibration could be dispensed with. (For a truly ideal calorimeter, it will also be unbiased, so $J_{2}=B_{2}=0$. Then, only a single run with known $C_{p} \neq 0$ is needed to find $J_{1}$ and $B_{1}$.)

The lack of accuracy for the measurement of the temperature of the sample has three possible drawbacks.

- The cyclic heat flow, i.e. $\hat{Q}$, is given by $\hat{C} \hat{T}_{s}\left(=C_{p} \hat{T}_{s}\right.$ for the standard inert case $)$ and is then only known if $\hat{T}_{s}$ is known. This is a minor difficulty as it tends to be $\hat{C}=\hat{Q} / \hat{T}_{s}$ which is of interest, as this gives information about heat capacities and temperature dependencies of kinetic processes.

- The relation between $\hat{C}$ and $\Delta \hat{T}$ is non-linear, because it can be expected that $K_{3}$ is nonzero. Again, this should cause no real problems because $K_{3}$ is determined through the extra calibration run and a cyclic measurement such as

$$
\hat{C}=\frac{(\Delta \hat{T} / B)+\left(i K_{1}\right)}{\left(-i K_{2}\right)-K_{3}(\Delta \hat{T} / B)}
$$

can then be used.

- For measurements of temperature-dependent properties of a sample, it is how things vary with $T_{s} \simeq \bar{T}_{s}$, not with $T_{m}$ or $\bar{T}_{m}$, which are really important. It can generally be expected that the difference is small enough for events which occur when $T_{m}=T^{*}$ to be interpreted as happening when $T_{s}=T^{*}$ but in the case of a phase transition this is not so clear. (In these more extreme processes it is obviously important that no significant variation of the thermal properties of the calorimeter occur over the range of temperatures during the events.) To allow for this, how the actual underlying temperature of the sample relates to $\bar{T}_{m}=T_{0}+\beta t$ and the measurement $\Delta \bar{T}$ needs to be found: with $\bar{T}_{s}=\bar{T}_{m}+J_{3} \Delta \bar{T}$, some form of calibration is needed to determine $J_{3}$. One possibility is to use a sample with known transition, for instance a glass transition: $J_{3}=\left(T_{g}-T_{g m}\right) / \Delta \bar{T}$ if a glass transition which is known to occur at $T_{g}$ appears to take place at $T_{g m}$. A variant of this might be to use a sample which, although free of transition, 
does have significant but known variation of heat capacity with temperature (the properties of the calorimeter should vary less significantly). Now:

$\beta C_{p}\left(\bar{T}_{s}\right)=J_{1} \Delta \bar{T}+J_{2} \beta$ with $\bar{T}_{s}=\bar{T}_{m}+J_{3} \Delta \bar{T}$

(neglecting any modulation) and for a "slow" ramp (so temperature variations in the device are small enough for $C_{p}$ to be assumed to have locally linear dependence upon temperature),

$$
\beta C_{p}\left(\bar{T}_{m}\right)+\beta \frac{d C}{d T}\left(\bar{T}_{m}\right) J_{3} \bar{T} \cong J_{1} \Delta \bar{T}+J_{2} \beta
$$

The bias $J_{2} / J_{1}$ can be found, as before, by an empty run, and then from two further runs, with different sized samples, $J_{1}$ and $J_{3}$ may be determined. (It might be noted that should the first derivative $\frac{d C_{p}}{d T}$ be noticeable, improved accuracy in a cyclic calibration should be given by replacing $C_{p}$ by $\hat{C}=C_{p}-\frac{i \beta}{\omega} \frac{d C_{p}}{d T}$, since:

$$
\frac{d Q}{d t}=(\beta+B \omega \cos \omega t) C_{p}\left(T_{0}+\beta t+B \sin \omega t\right) \simeq \beta C_{p}+B\left(\omega C_{p} \cos \omega t+\beta \frac{d C_{p}}{d T} \sin \omega t\right)
$$

All the $a$ 's appearing in the relationships between heat flows and temperatures depend, as noted earlier, upon frequency in some generally unknown way which means that ideally calibrations should be done at the frequency of an experiment. One thing, however, is clear, as $\omega$ is reduced towards zero (so the modulation gets more like a ramp), the coefficients in the equations for $\hat{T}$ approach those in the earlier equations involving $\hat{T}$ (scaled by an appropriate power of $\omega$ ). This means that the calibration factors and constants tend to limiting values as $\omega$ is reduced to zero: extrapolation of uncorrected results can lead to measurements for $C_{p}$ improved over the basic (uncorrected) values.

One further difficulty is the variation in thermal contract between calorimeter and pan (and/or between pan and sample) from run to run. Hatta [21,22] produced a method to account for varying thermal resistance taking an inert sample ( $C_{p}$ real and positive) and a simple model for a calorimeter. The same method can be extended and combined with the above general model to account for an uncertain heat-transfer coefficient between the sample and its pan, but 
assuming good thermal contact between the pan and the calorimeter. (If heat-transfer coefficients between the pan and its contents and between the pan and its environs are both unknown - and finite - correction will be significantly harder.)

Replacing $T_{s}$ by the pan's temperature $T_{p}$, the above procedure using cyclic temperature measurements can be used to obtain an overall heat capacity measurement for the sample and its pan:

$C_{p s}=\frac{(\Delta \hat{T} / B)+i K_{1}}{\left(-i K_{2}\right)-K_{3}(\Delta \hat{T} / B)}$

Now focusing on the pan and sample,

$C_{p} \frac{d T_{s}}{d t}=K_{I}\left(T_{p}-T_{s}\right), \quad \dot{Q}=C_{s p} \frac{d T_{p}}{d t}+C_{p} \frac{d T_{s}}{d t}$

where $\dot{Q}$ is the heat flow into the pan from the calorimeter, $C_{s p}$ is the heat capacity of the pan and $K_{I}$ is the heat-transfer coefficient between sample and pan. The modulated parts are then related through:

$\left(K_{I}+i \omega C_{p}\right) \hat{T}_{s}=K_{I} \hat{T}_{p}, \quad i \omega C_{p s} \hat{T}_{p}=i \omega C_{s p} \hat{T}_{p}+i \omega C_{p} \hat{T}_{s}$

so,

$\frac{1}{C_{p s}-C_{s p}}=\frac{1}{C_{p}}+\frac{i \omega}{K_{I}}$

(146)

Where the sample is inert (before, after and between transitions), $C_{p}$ is real and positive, as is $C_{s p}$, the heat capacity of the actual pan (also assumed known). The real and imaginary parts can then be used to determine $C_{p}$ and $K_{I}$. During a transition $C_{p}$ is no longer real, but could still be found if $K_{I}$ were known. One approach is then to interpolate for $K_{I}$ between its values before and after the transition.

Of course during calibration the $K_{I}$ might again vary (and be finite). This complicates the initial determination of $K_{1}, K_{2}, K_{3}$. It seems likely that now six runs could be needed: giving a total of twelve (real) pieces of data (from the real and imaginary parts) to fix the three complex constants $K_{1}, K_{2}$ and $K_{3}$ (six real bits of information) and six different $K_{I}$ 's, all real although these are not really wanted. 
The above discussion outlines how it is possible, in principle, to deal with almost all of the nonidealities of real world DSC cells with sufficient ingenuity and effort. It is probable that commercial instruments will gradually implement these procedures so that they will become available to many scientists. However, not considered above are the problems that arise from poor thermal conductivity within the sample. Once calibrated, the different thermal resistances within the calorimeter should behave in a predictable way. Of course this is not true of the sample. We are not talking about measuring the thermal conductivity of the sample while no transition is occurring, which has already been done [47] by MTDSC, but doing so while a transition is occurring and, preferably, also measuring the reversing and non-reversing signals as well. This remains a challenge for the future. For the present, most workers are best advised to use relatively long periods, of the order of 60 seconds, as this reduces the effects of thermal resistances.

\section{REFERENCES}

[1] S.L .Simon, Thermochim.Acta, 374 (2001) 55.

[2] M. Reading, D. Elliott and V.L. Hill, Proc. NATAS (1992) 145.

[3] P.S. Gill, M. Reading, I.M. Salin and J.C. Seferis, Proc. Greek Acad. Sci., 67, (1992) 311.

[4] M. Reading, B.K. Hahn and B.S. Crowe, US patent 5,224,775 (1993).

[5] M. Reading, Trends Polym. Sci., 1 (1993) 8.

[6] M. Reading, D Elliott and V.L.Hill, J. Thermal Anal., 40 (1993) 949.

[7] P.S. Gill, S.R. Sauerbrunn and M. Reading, J. Thermal Anal., 40 (1993) 939.

[8] M. Reading, R. Wilson and H.M. Pollock, Proc. NATAS (1994) 2.

[9] M. Reading, A. Luget and R. Wilson, Thermochim. Acta, 238 (1994) 295.

[10] M. Reading, US Patent 5,474,385 (1995).

[11] M. Song, A. Hammiche, H.M. Pollock, D.J. Hourston and M. Reading, Polymer, 36 (1995) 3313.

[12] A.A. Lacey, C. Nikolopoulos and M. Reading, J. Thermal Anal., 50(1-2) (1997) 279.

[13] D.J. Hourston, M. Song, A. Hammiche, H.M. Pollock and M. Reading, Polymer, 38 (1997) 1.

[14] K.J. Jones, I. Kinshott, M. Reading, A.A. Lacey, C. Nikolopoulos and H.M. Pollock, Thermochim. Acta, 305 (1997) 187.

[15] M. Reading, Thermochim. Acta, 292 (1997) 179.

[16] M. Reading and R. Luyt, J. Thermal Anal. Cal., 54 (1998) 535.

[17] M. Reading, J. Thermal Anal. Cal., 54 (1998) 411.

[18] J.E.K. Schawe, Thermochim. Acta, 261, (1995) 183.

[19] P.J. Haines, M. Reading, F.W.Wilburn, in: M.E. Brown (Ed.), Handbook of Thermal Analysis and Calorimetry, Volume 1: Principles and Practice, Ch. 5, Elsevier Science 
B.V., Amsterdam (1998).

[20] R. Androsch and B. Wunderlich, Thermochim. Acta, 333 (1999) 27.

[21] I. Hatta and S. Muramatsu, Jap. J. Appl. Phys., 35 (1996) L858.

[22] I. Hatta and N. Katayama, J. Thermal Anal., 4 (1998) 577.

[23] A. Toda, C. Tomita, M. Hikosaka and Y. Sarayuma, Polymer, 39 (1998) 5093.

[24] I. Hatta and A.A. Minakov; Thermochim. Acta, 330 (1999) 39.

[25] A. Toda, T. Arita, C. Tomita and M. Hikosaka; Polymer, 41 (2000) 8941.

[26] I. Hatta and S. Muramatsu, Jpn. Jl. Appl. Phys., 35 (1996) L858.

[27] I. Hatta and N. Katayama, J. Thermal Analysis, 4 (1998) 577

[28] A.K. Galwey and M.E. Brown, in: M.E. Brown (Ed.), Handbook of Thermal Analysis and Calorimetry, Volume 1: Principles and Practice, Ch. 3, Elsevier Science B.V., Amsterdam (1998).

[29] A. Toda, T. Arita and M. Hikoska, J. Thermal Anal. Cal., 60 (2000) 821.

[30] M. Reading, K.J. Jones and R. Wilson, Netsu Sokutie, 22 (1995) 83.

[31] B. Wunderlich, in E.A. Turi (Ed.), Thermal Characterization of Polymeric Materials, Vol. 1, Ch.2, Academic Press, San Diego (1997).

[32] J.M. Hutchinson and S. Montserrat, Thermochim. Acta, 377 (2001) 63.

[33] J.M. Hutchinson, A. Boon Tong and Z. Jiang, Thermochim. Acta, 335 (1999) 27.

[34] S. Montserrat, J. Polym. Sci. B Polym. Phys., 38 (2000) 2272.

[35] D.J. Hourston, M. Song, A. Hammiche, H.M. Pollock and M. Reading, Polymer. 37 (1996) 243.

[36] N.A. Bailey, J.N. Hay and D.M. Price, Thermochim. Acta, 367-368 (2001) 425.

[37] M. Reading, D.M. Price and H. Orliac, in A.T. Riga and L.H. Judovits (Eds), Material Characterization by Dynamic and Modulated Thermal Analytical Techniques, ASTM STP 1402 American Society for Testing and Materials, West Conshohocken, PA (2001) pp. 17-31.

[38] S.M. Sarge, E. Gmelin, G.W.H. Höhne, H.K. Cammenga, W. Hemminger and W. Eysel, Thermochim. Acta, 247 (1994) 129-168.

[39] S. Weyer, A. Hensel and C. Schick, Thermochim. Acta ,305 (1997) 267.

[40] W.P. Brennan, B. Miller and J.C. Whitwell, Ind. Eng. Chem. Fundam. 8 (1969) 314.

[41] J.M. Hutchinson and A.J. Kovacs, J. Polym. Sci. B Polym. Phys., 14 (1976) 1575.

[42] A. Toda, T. Oda, M. Hikosaka and Y. Saruyama, Thermochim Acta, 293 (1997) 47.

[43] A. Toda, T. Oda, M. Hikosaka and Y. Surayama, Polymer, 38 (1997) 231

[44] B. Wunderlich; "Macromolecular Physics, Vol. 3: Crystal Melting", Academic Press, New York (1980).

[45] A.A. Lacey and C. Nikolopoulos, IMA Jl. Appl. Maths., 66 (2001) 449.

[46] B. Wunderlich, Y.M. Jin and A. Boller, Thermochim. Acta, 238 (1994) 277.

[47] S.M. Marcus and M. Reading, US Patent 5,335,993 (1994). 